\title{
ON APPROXIMATING CERTAIN INTEGRALS BY SUMS
}

BY

\author{
C. RAYMOND ADAMS AND ANTHONY P. MORSE
}

1. Introduction; the main problem and its setting. In a recent paper( ${ }^{(1)}$ we showed that if $E$ is a measurable linear set (bounded or unbounded), if $f \in L(E)$, if $k$ is a fixed real number $\geqq 1$, and if $A_{n}$ and $B_{n}(n=1,2,3, \cdots)$ are sequences (finite or infinite) of measurable subsets of $E$ satisfying the conditions

$$
\begin{gathered}
A_{n} \subset B_{n}, \quad 0<\left|B_{n}\right| \leqq k\left|A_{n}\right|, \quad \text { diameter }\left(B_{n}\right)<\delta \quad(n=1,2,3, \cdots) ; \\
\left|B_{m} B_{n}\right|=0 \text { for } m \neq n ; \quad \sum_{n} B_{n}=E,
\end{gathered}
$$

then the following relation holds:

$$
\int_{E} f=\lim _{\delta \rightarrow 0} \sum_{n}\left[\int_{A_{n}} f /\left|A_{n}\right|\right]\left|B_{n}\right| .
$$

The present paper is concerned chiefly with the generalization of this result obtained by replacing $f(x)$ by $\phi[f(x)]$. From the statement above it is evident that the order of the elements of the sequences $A_{n}$ and $B_{n}$ $(n=1,2,3, \cdots)$ is immaterial. It is therefore desirable to introduce a notation which will be free from any implication of order. To achieve this purpose and to enable us to state our present problem with precision we formulate several definitions as follows.

(1.1) Definition. For $B$ a non-vacuous linear set the diameter of $B$ is $\sup B-\inf B$; this is designated by the symbol diam $B$. For $B$ vacuous diam $B$ is defined as zero.

It will presently be clear that $\operatorname{diam} B$ can be interpreted as the essential diameter of $B$ without disturbing any of the subsequent results, where by essential diameter of $B$ is meant ess $\sup B$-ess inf $B$ when $|B|$ is $>0$ and zero when $|B|=0$.

Presented to the Society September 8, 1942; received by the editors August 22, 1942.

(1) Adams and Morse, Random sampling in the evaluation of a Lebesgue integral, Bull. Amer. Math. Soc. vol. 45 (1939) pp. 442-447, Theorem 4. Hereinafter this paper will be referred to as RS.

A set of real numbers will be spoken of as a linear set. For $E$ a linear set measurable in the sense of Lebesgue and of positive measure, $L(E)$ represents the class of functions $f$ the domain of each of which is $E$ and for each of which the Lebesgue integral $\int_{E} f(x) d x$ exists (finite). For $f \in L(E)$ we usually abbreviate $\int_{E} f(x) d x$ to $\int_{E} f$.

When and only when a linear set $E$ is measurable in the sense of Lebesgue its measure is designated by $|E|$.

All functions considered in this paper are real-valued. 
(1.2) Definition. For $E$ a measurable linear set and $0<\delta \leqq \infty a$-partition $F$ of $E$ is a countable family of measurable, essentially disjoint sets satisfying the conditions

$$
\sum_{B \in F} B=E, \quad \operatorname{diam} B<\delta \quad \text { for } B \in F .
$$

$F$ will be spoken of as an infinite family if and only if the sets $B \in F$ are infinite in number. The aggregate of all $\delta$-partitions of $E$ will be represented by $\Gamma_{\delta}(E)$.

(1.3) Definition. For $E$ a measurable linear set $L^{*}(E)$ is the class of functions defined by the condition $f \in L^{*}(E)$ if and only if the domain of $f$ is $E$ and the condition

$$
-\infty<\int_{B} f<\infty
$$

is satisfied for each measurable subset $B \subset E$ with

$$
|B|>0, \quad \operatorname{diam} B<\infty \text {. }
$$

Clearly we have $L(E) \subset L^{*}(E)$, with $L(E)=L^{*}(E)$ if and only if $E$ is essentially bounded.

(1.4) Definition. For $f \in L^{*}(E)$ and $B$ a measurable subset of $E$ with $0<|B|<\infty$ we define

$$
\mathfrak{M}_{B} f=\int_{B} f /|B|
$$

The set of numbers of the form $\mathfrak{M}_{B} f$, where $B$ is a measurable subset of $E$ of positive measure with diam $B<\infty$, will be designated by $\Re(f)$ and the closure of this set by $\overline{\Re(f)}$.

It is easily seen that when $|E|$ is $>0, \Re(f)$ is an interval, and that this interval may be open, semi-open, or closed, as well as bounded or unbounded; the end points of this interval, whether finite or infinite, are ess $\inf _{x \in E} f(x)$ and ess $\sup _{x \in E} f(x)$. The subset of $E$ on which $f$ is not in $\Re(f)$ is of measure zero.

(1.5) Definition. For $f \in L^{*}(E)$ and $\phi$ a function whose domain includes $\Re(f)$ we interpret

$$
\phi\left[\mathfrak{M}_{B} f\right]|B| \quad \text { for } B \subset E,|B|=0,
$$

as zero. For $0<\delta \leqq \infty$ and $F \in \Gamma_{\delta}(E)$ we assign to the numerical sum

$$
\sum_{B \in F} \phi\left[\mathfrak{M}_{B} f\right]|B|
$$

its natural intuitional meaning (to be made precise later in Definition (2.7)) and 
define two limits, which are actually of the nature of a lower integral and an upper integral respectively over $E$, as follows:

$$
\begin{aligned}
& S_{*}(f, \phi, E)=\lim _{\delta \rightarrow 0} \inf _{F \in \Gamma_{\delta}(E)} \sum_{B \in F} \phi\left[\mathfrak{M}_{B} f\right]|B|, \\
& S^{*}(f, \phi, E)=\lim _{\delta \rightarrow 0} \sup _{F \in \mathrm{r}_{\delta}(E)} \sum_{B \in F} \phi\left[\mathfrak{M}_{B} f\right]|B| .
\end{aligned}
$$

That each of these limits exists (finite or infinite) follows from the fact that

$$
0<\delta_{1} \leqq \delta_{2} \leqq \infty \text { implies } \Gamma_{\delta_{1}}(E) \subset \Gamma_{\delta_{2}}(E) .
$$

When and only when these limits are the same we define

$$
-\infty \leqq S(f, \phi, E)=S_{*}(f, \phi, E)=S^{*}(f, \phi, E) \leqq \infty .
$$

Similarly we define two other limits, involving the sampling procedure, for any fixed real number $k \geqq 1: S_{*}(f, \phi, E, k) \quad\left[S^{*}(f, \phi, E, k)\right]$ is the $\lim _{\delta \rightarrow 0}$ of the inf [sup] of numbers of the form

$$
\sum_{B \in F} \phi\left[\mathfrak{M}_{A B f}\right]|B|,
$$

where $F \in \Gamma_{\delta}(E)$ and $A$ is a measurable linear set variable with $F$ and for each $F$ variable within the restriction

$$
|B| \leqq k|A B| \quad \text { for } B \in F .
$$

If and only if these limits are the same we write

$$
-\infty \leqq S(f, \phi, E, k)=S_{*}(f, \phi, E, k)=S^{*}(f, \phi, E, k) \leqq \infty .
$$

(1.7) Definition. For $E$ a linear set, $f$ a function whose domain is $E$, and $\phi$ a function whose domain includes the range of $f$, we represent by $\phi: f$ the function $g$ defined on $E$ by the condition

$$
g(x)=\phi[f(x)] \quad \text { for } x \in E .
$$

Our main problem is to determine conditions on $f$ and $\phi$ which will insure the (finite) existence of $\int_{E} \phi: f$ and of $S(f, \phi, E, k)$ and the equality

$$
\int_{E} \phi: f=S(f, \phi, E, k) \text {. }
$$

Actually the conditions which we obtain will insure

$$
\begin{aligned}
\lim _{\delta \rightarrow 0} \sup _{F \in \Gamma_{\delta}(E)} \sum_{B \in F} \mid & \int_{B} \phi: f-\phi\left[\mathfrak{M}_{A B} f\right]|B| \mid \\
& =\lim _{\delta \rightarrow 0} \sup _{r \in \Gamma_{\delta}(E)} \sum_{B \in F}\left|\mathfrak{M}_{B} \phi: f-\phi\left[\mathfrak{M}_{A B} f\right]\right||B|=0,
\end{aligned}
$$

which implies (1.8).

For convenience of reference we attach numbers to the following particu- 
lar cases of (1.8) and (1.9) in which no sampling is involved:

$$
\begin{gathered}
\int_{E} \phi: f=S(f, \phi, E), \\
\lim _{\delta \rightarrow 0} \sup _{F \in \Gamma_{\delta}(E)} \sum_{B \in F}\left|\mathfrak{M}_{B} \phi: f-\phi\left[\mathfrak{M}_{B} f\right]\right||B|=0 .
\end{gathered}
$$

The result quoted above from RS shows that the conditions $f \in L(E)$, $\phi(y)=y$ for $y \in \Re(f)$ are sufficient for (1.8). In RS the condition (1.9) could equally well have been obtained, but it was not. It has also been shown by Banach ( $\left.{ }^{2}\right)$ that if $E=I$, the unit interval $0 \leqq x \leqq 1$, and if the sets $B \in \Gamma_{\delta}(I)$ are restricted to be a finite set of intervals, the conditions $f \in L_{p}(I), p>1$, $\phi(y)=|y|^{p}$ for $y \in \Re(f)$ are sufficient for (1.10).

It should be noted that if $\phi$ is regarded as defined on the infinite interval $-\infty<y<\infty$ and fixed, the subclass of $L(E)$ singled out by the condition $\int_{E} \phi: f$ exists may naturally be called $L_{\phi}(E)$, thus generalizing the familiar $L_{p}$ classes of functions. This is by no means the first time that such generalizations have been considered. In fact they were introduced as early as 1924 by W. H. Young $\left({ }^{3}\right)$ who used the term "super-summability" in this connection. This notion was further developed, and related problems studied, by Young and by others including Burkill, Kaczmarz and Nikliborc, Orlicz, and Birnbaum and Orlicz $\left({ }^{4}\right)$. There is no need to describe here these earlier investigations; it is sufficient to remark that the objectives of their authors were quite different from ours and were such as to require hypotheses on the function $\phi$ more restrictive than those which we shall impose.

2. Further definitions and preliminaries. We collect here a number of definitions which are to be used more or less generally throughout this paper. Other definitions, of only local use in the subsequent developments, will be formulated when needed.

(2.1) Definition. If $Q$ is a condition involving the symbol $a, E_{a}[Q]$ stands for the set defined by the condition $b \in E_{a}[Q]$ if and only if $Q$ is satisfied when $b$ is substituted for a.

(2.2) Definition. If $A$ is $a$ set and $a \in A$ we define $\{a\}$ as $E_{x}[x=a]$.

(2) Banach, Sur les opérations dans les ensembles abstraits et leur application aux équations intégrales, Fund. Math. vol. 3 (1922) pp. 133-181; especially pp. $175-177$. We use $L_{p}(E)$ to stand for the class of functions $f$ with $f \in L(E),|f|^{p} \in L(E)$.

(3) W. H. Young, The progress of mathematical analysis in the twentieth century, Proc. London Math. Soc. (2) vol. 24 (1925-1926) pp. 421-434.

(4) Burkill, The strong and weak convergence of functions of general type, Proc. London Math. Soc. (2) vol. 28 (1928) pp. 493-500; Kaczmarz and Nikliborc, Sur les suites des fonctions convergentes en moyenne, Fund. Math. vol. 11 (1928) pp. 151-168; Orlicz, Beiträge zur Theorie der Orthogonalentwicklungen, Studia Mathematica vol. 1 (1929) pp. 1-39, 241-255; Birnbaum and Orlicz, Über Approximation im Mittel, ibid. vol. 2 (1930) pp. 197-206, and Über die Verallgemeinerung des Begriffes der zueinander konjugierten Potenzen, ibid. vol. 3 (1931) pp. 1-67. 
(2.3) Definition. The symbol $\sim$, placed above another symbol, negates the meaning of the other symbol; in particular, if $A$ is a set, $a \tilde{\varepsilon} A$ means that $a$ is not an element of $A$.

(2.4) Definition. For $a$ and $b$ real numbers with $a \leqq b$ we define $[a, b]$ as the closed interval $E_{x}[a \leqq x \leqq b]$; for $a<b$ the open interval $E_{x}[a<x<b]$ is represented by $(a, b)$. The unit interval $[0,1]$ is designated by $I$.

(2.5) Definition. If $F$ is a family of sets $B$, we sometimes employ for the set $\sum_{B \in F} B$ the abbreviation $\sigma(F)$.

It should be noted that $x \in \sigma(F)$ if and only if there exists $B \in F$ with $x \in B$.

(2.6) Definition. Two sets $A$ and $B$ are called disjoint if and only if $A B$ is vacuous (that is, $A B=0$ ). $A$ family $F$ of sets $B$ is called disjointed if and only if $B_{1} B_{2}=0$ whenever $B_{1} \in F, B_{2} \in F$ and $B_{1} \neq B_{2}$.

Adopting the convention that $a+\infty=\infty, a-\infty=-\infty$ when $-\infty<a<\infty$ and that $\infty-\infty$ is meaningless, we set up the following formal definition for numerical sums.

(2.7) Definition. If $H$ is a countable set with $N(\leqq \infty)$ elements and we have $-\infty \leqq a_{x} \leqq \infty$ for $x \in H$, the numerical sum $\sum_{x \in H} a_{x}$ is the unique number $\lambda$ $(-\infty \leqq \lambda \leqq \infty)$, if any exists, such that

whenever

$$
\lambda=\sum_{j=1}^{N} a_{x_{j}}
$$

$$
H=\sum_{j=1}^{N}\left\{x_{j}\right\} \quad \text { with } x_{m} \neq x_{n} \text { for } 1 \leqq m<n<N+1 .
$$

If $H$ is vacuous we understand

$$
\sum_{x \in H} a_{x}=0 .
$$

It should be noted that $\left|\sum_{x \in H} a_{x}\right|<\infty$ implies $\sum_{x \in H}\left|a_{x}\right|<\infty$.

(2.8) Definition. For $J$ a linear interval with $0<|J| \leqq \infty$ a $\delta$-partition of $J$ in which each set $B$ is an interval (open, semi-open, or closed) with $|B|>0$ is called an interval $\delta$-partition of $J$; the aggregate of all such interval $\delta$-partitions is represented by $\Gamma_{\delta}(J)$; and limits corresponding to those of Definition (1.5) are designated by

$$
\bar{S}_{*}(f, \phi, J, k), \quad \bar{S}^{*}(f, \phi, J, k), \text { and so on. }
$$

(2.9) Definition. For $E$ a linear set the symbols $C(E), U C(E)$, and $B(E)$ are used to designate, respectively, the classes of functions $f$ the domain of each 
of which is $E$ and each of which is continuous on $E$, uniformly continuous on $E$, and bounded on $E . R(I)$ stands for the class of functions each of which is (properly) Riemann integrable on $I ; R^{*}(I)$ for the class of functions defined thus: $f \in R^{*}(I)$ if and only if for each $N>0, f_{N} \in R(I)$ where $f_{N}(x)=f(x)$ for $x \in E_{x}[-N \leqq f(x) \leqq N], f_{N}(x)=-N$ for $x \in E_{x}[f(x)<-N], f_{N}(x)=N$ for $x \in E_{x}[f(x)>N]$, and $\lim _{N \rightarrow \infty} \int_{I} f_{N}$ exists (finite). The notation $B C(E)$ [or $B U C(E)$ or $B L_{\phi}(E)$ or $\left.\cdots\right]$ indicates the intersection of the classes $B(E)$ and $C(E)$ or $U C(E)$ or $L_{\phi}(E)$ or . . ]. The condition $f \in L(I)-R(I)$ means that $f$ is an element of $L(I)$ but not of $R(I)$. If $A$ stands for any one of the classes mentioned here the relation $\phi \in A(E)$ is to be interpreted as meaning that $\phi$ is a function of the class $A$ on $E$; in contrast, the relation $f \in A(E)[\phi: f \in A(E)]$ is understood to mean that $f[\phi: f]$ is almost everywhere on $E$ equal to a function of the class $A$ on $E$.

In $\$ 3$ we prove some theorems in the theory of functions of sets. These considerations are made in a generality considerably greater than is necessary for our particular purposes, since they seem to us to be fundamental to that theory and to possess some intrinsic interest. They are applied in $\$ 4$, where we determine certain properties of the sums that appear in Definition (1.5).

In $\$ 5$ we prove a sequence of theorems of which Theorem (5.12), the climax, gives a necessary and sufficient condition for (1.9) when $f \in L^{*}(E)$ and $\phi \in C(\Re(f))$. A sufficient condition, in terms of the existence of a convex dominant for $|\phi|$ and of such nature as frequently to be useful in testing, is given in Theorem (5.13). The necessity of this condition is the subject of inquiry in $\$ \S 7,8$ following the establishment of a number of preliminary lemmas in $\$ 6$.

Obviously the condition $f \in L^{*}(E)$ is necessary for the mere formulation of the main questions as expressed by (1.8), (1.9), (1.10), and (1.11). The object of $\S 9$ is to show that the hypothesis $\phi \in C(\Re(f))$ is also essential. In $\$ 10$ we analyze the circumstances under which the $k$-hypothesis on the sample sets can be relaxed; that is, the condition (1.6) replaced by

$$
|A B|>0 \quad \text { for } B \in F \in \Gamma_{\delta}(E) \text {. }
$$

In $\$ 11$, we apply some of the earlier results to functions which are of bounded variation or are absolutely continuous in a certain generalized sense.

Our results hold also for a measurable set $E$ in Euclidean $n$-space. Even in $\$ 6$ where the treatment appears to be peculiarly 1-dimensional, one need only alter Definition (6.1) to employ $n$-dimensional measure in connection with the function $u$ (and later $f$ ) and linear measure in connection with the equimeasurable function $v$ (and later $g$ ).

In general definitions, theorems, and displayed relations to which other than local reference is made will be numbered sequentially in a decimal sys- 
tem with the first number indicating a section. A displayed relation referred to only in the proof where it occurs will be labeled with a letter, such as (a).

3. Some theorems in the theory of functions of sets. In this section the symbol 0 will always stand for the vacuous set. We understand that two sets $E_{1}$ and $E_{2}$ are identical, and write $E_{1}=E_{2}$, if and only if $x \in E_{1}$ implies and is implied by $x \in E_{2}$; otherwise we call the sets distinct and write $E_{1} \neq E_{2}$. Under this definition of distinctness there is only one vacuous set. An aggregate of sets will usually be spoken of as a family.

(3.1) Definition. A family (S) is called $a \sigma$-field if and only if (i) $H$ a countable subfamily of (B) implies $\sigma(H) \in(S$, and (ii) $B \in(B)$ implies $\sigma(\mathbb{B})-B \in(B$.

If $(B)$ is an arbitrary family, $\beta \in B$ implies $\beta \subset \sigma(B)$.

If $(S)$ is a $\sigma$-field, (i) implies $0=\sigma(0) \in(B ;$; from (ii) then follows $\sigma(\$) \in(B$; whence we find that $B_{1} \in\left(S, B_{2} \in(S)\right.$ imply $B_{1} B_{2} \in(S)$ and $B_{2}-B_{1}=B_{2}-B_{1} B_{2} \in(S$.

The simplest and "smallest" $\sigma$-field is $\{0\}$. If $S$ represents Euclidean $n$-space $(1 \leqq n<\infty)$ and $E \subset S$ is a measurable set (of finite or infinite measure in the sense of Lebesgue), the measurable subsets of $E$ constitute a $\sigma$-field (s) with $\sigma(B)=E$.

(3.2) Definition. If $A$ and $B$ are sets, we define

$$
A * B=A \underset{\beta}{E}[\beta \subset B] \text {. }
$$

The following theorem may easily be verified.

(3.3) Theorem. If $(S)$ is a $\sigma$-field and $B \in(\$$, then $B * B$ is a $\sigma$-field with $\sigma(B) B)=B$.

(3.4) Definition. For (S) $a$-field $F$ is called $a$ (\$-partition if and only if $F$ is a countable disjointed subfamily of (S) with $\sigma(F)=\sigma(\mathbb{S})$. The aggregate of all (5)-partitions is represented by (5) ${ }^{2}$.

It may be noted that if $B^{3}$ is a $\sigma$-field, $\{\sigma(B)\} \in\left(B^{*}\right.$; thus $\left(B^{*}\right.$ is never vacuous. Also $F \in(5 \pi$ implies $F-\{0\} \in(S \pi$.

(3.5) Definition. If $B$ is a $\sigma$-field and $f$ a function whose domain includes (\$), $f$ is said to be quasi-additive on $(\xi)$ when and only when

$$
\sup _{F \in \Im^{\pi}}\left|\sum_{B \in F} f(B)\right|<\infty .
$$

A well known theorem on the summation of absolutely convergent double series yields a result which we find convenient for our present purposes to put in the following form.

(3.6) Theorem. If $F$ is a countable family and for $B \in F, K_{B}$ is a countable 
family; $K_{B_{1}} K_{B_{2}}=0$ whenever $B_{1} \in F, B_{2} \in F, B_{1} \neq B_{2} ; S=\sum_{B \in F} K_{B}$; and $f$ is a function whose domain includes $S$ and which satisfies the condition $\left|\sum_{\beta \in s} f(\beta)\right|$ $<\infty$ (and therefore $\left.\sum_{\beta \in S}|f(\beta)|<\infty\right)$, then

$$
\sum_{\beta \in S} f(\beta)=\sum_{B \in F} \sum_{\beta \in K_{B}} f(\beta) .
$$

In the rest of this section frequent use will be made of this theorem.

(3.7) TheOrem. If $f$ is quasi-additive on (S) and $B \in \mathbb{S}$, then $f$ is quasiadditive on $(5) * B$.

Proof. Let $E=\sigma(\mathbb{S})$. In case $E-B=0$ we have $(\$) * B=(B) * E=(\$) * \sigma(\mathbb{B})$ $=\&$ and the theorem is trivially true. If $E-B \neq 0$, we let $F$ be an arbitrary element of $(B) * B)^{\pi}$, set $F^{\prime}=\{E-B\}+F$, note that $F^{\prime} \in(5)$, and observe that the relation

$$
\begin{aligned}
\left|\sum_{\beta \in F} f(\beta)\right| & \leqq|-f(E-B)|+\left|f(E-B)+\sum_{\beta \in F} f(\beta)\right| \\
& =|f(E-B)|+\left|\sum_{\beta \in F^{\prime}} f(\beta)\right| \leqq|f(E-B)|+\sup _{F^{\prime \prime} \in \mho^{\pi}}\left|\sum_{\beta \in F^{\prime \prime}} f(\beta)\right|
\end{aligned}
$$

completes the proof.

(3.8) Lemma. If $S$ is quasi-additive on (\$), $A \in \mathbb{B}, B \in \mathbb{S}, A+B=\sigma(\mathbb{S})$, $A B=0$, and $f$ is bounded on $(\mathbb{S} * A$ and on $\mathbb{B} * B$, then $f$ is bounded on $(\mathbb{S}$.

Proof. Let $E=\sigma(\$)$,

$$
N=\sup _{F \in \mathbb{G}^{\pi}}\left|\sum_{\beta \in F} f(\beta)\right|, \quad N_{A}=\sup _{\beta \in \mathbb{G}_{*} A}|f(\beta)|, \quad N_{B}=\sup _{\beta \in \mathbb{S}_{*} B}|f(\beta)| .
$$

Let $\gamma^{\prime}$ be an arbitrary element of $(\xi), \alpha^{\prime}=A\left(E-\gamma^{\prime}\right), \beta^{\prime}=B\left(E-\gamma^{\prime}\right)$, and note that $\alpha^{\prime} \beta^{\prime}=\beta^{\prime} \gamma^{\prime}=\gamma^{\prime} \alpha^{\prime}=0$. If $\alpha^{\prime}+\gamma^{\prime}=0$, the relation

$$
\left|f\left(\gamma^{\prime}\right)\right| \leqq N+\left|f\left(\alpha^{\prime}\right)\right|+\left|f\left(\beta^{\prime}\right)\right| \leqq N+N_{A}+N_{B}
$$

is apparent; if $\alpha^{\prime}+\gamma^{\prime} \neq 0$ and $\beta^{\prime}=0$, it is a consequence of the inequality

$$
\left|f\left(\boldsymbol{\alpha}^{\prime}\right)+f\left(\boldsymbol{\gamma}^{\prime}\right)\right| \leqq N \text {; }
$$

and if $\alpha^{\prime}+\gamma^{\prime} \neq 0$ and $\beta^{\prime} \neq 0$, it follows from the inequality

$$
\left|f\left(\alpha^{\prime}\right)+f\left(\beta^{\prime}\right)+f\left(\gamma^{\prime}\right)\right| \leqq N .
$$

Thus the proof is complete.

(3.9) Lемма. If $f$ is quasi-additive but unbounded on $\$$, there exist sets $A$ and $B$ with

$$
A \in \text { (), } B \in \text { (\$, } \quad A+B=\sigma(\$), \quad A B=0, \quad|f(A)|>1+|f(0)|,
$$


$f$ unbounded on (S) $* B$.

Proof. Let $E$ and $N$ have the meaning assigned them in the last proof. Since $f$ is unbounded on (B) we take $B_{0} \in(B)$ such that

$$
\left|f\left(B_{0}\right)\right|>N+1+|f(0)| \text {, }
$$

and let $A_{0}=E-B_{0}$. Clearly we have $B_{0} \neq 0$ and

$$
\begin{aligned}
A_{0} \in \text { S }, \quad B_{0} \in \text { S }, & A_{0}+B_{0}=E, \quad A_{0} B_{0}=0, \quad\left|f\left(B_{0}\right)\right|>1+|f(0)|, \\
\left|f\left(A_{0}\right)\right| & \geqq\left|f\left(B_{0}\right)\right|-\left|f\left(A_{0}\right)+f\left(B_{0}\right)\right| \\
& >N+1+|f(0)|-N=1+|f(0)| .
\end{aligned}
$$

From Lemma (3.8) we infer that $f$ is either unbounded on $B * B_{0}$ or unbounded on $(S) * A_{0}$; in the first alternative the desired conclusion is reached by taking $A=A_{0}$ and $B=B_{0}$, whereas in the second case it is obtained by taking $A=B_{0}$ and $B=A_{0}$.

A proof of a well known theorem on completely additive functions of sets $\left(^{(5)}\right.$, which was communicated to us some time ago by R. M. Robinson, has been of use to us at this juncture. The following is a generalization of that theorem.

(3.10) TheOREM. If $f$ is quasi-additive on $(\$), f$ is bounded on (\$).

Proof. We shall show that the assumption of the hypothesis and the contrary of the conclusion leads to a contradiction. Let $B_{0}=\sigma(\xi)$. In view of Theorem (3.7), Lemma (3.9), and Theorem (3.3) there exist sets $A_{1}, A_{2}, A_{3}, \cdots$ and $B_{1}, B_{2}, B_{3}, \cdots$ satisfying the following recursive condition: if $n$ is a positive integer and

$$
A_{n} \in \text { S } * B_{n-1}, \quad B_{n} \in \text { S } * B_{n-1}, \quad A_{n}+B_{n}=B_{n-1}, \quad A_{n} B_{n}=0,
$$

then we have

$$
\left|f\left(A_{n}\right)\right|>1+|f(0)|, \quad f \text { is unbounded on }(\xi) * B_{n} \text {, }
$$

$$
\begin{aligned}
& A_{n+1} \in \text { (S) } * B_{n}, \quad B_{n+1} \in \text { (S) } * B_{n}, \quad A_{n+1}+B_{n+1}=B_{n}, \quad A_{n+1} B_{n+1}=0, \\
& \left|f\left(A_{n+1}\right)\right|>1+|f(0)|, \quad f \text { is unbounded on }(\&) B_{n+1} \text {. }
\end{aligned}
$$

Clearly we also have

$$
\begin{gathered}
B_{0} \supset B_{1} \supset B_{2} \supset \cdots, \\
A_{m} B_{n} \subset A_{m} B_{n-1} \subset A_{m} B_{m}=0
\end{gathered}
$$

Defining

$$
A_{0}=B_{0}-\sum_{j=1}^{\infty} A_{j}, \quad F_{0}=\sum_{j=0}^{\infty}\left\{A_{j}\right\},
$$

we see that $A_{m} A_{n}=0$ for $0 \leqq m<n$. From this and the fact that

(5) See, for example, Saks, Theory of the integral, Warsaw, 1937, Theorem (6.1), p. 10. 


$$
\left|f\left(A_{n}\right)\right|>1+|f(0)| \text { implies } A_{n} \neq 0 \quad(n=1 ; 2,3, \cdots),
$$

we infer

$$
A_{m} \neq A_{n} \quad \text { for } 0 \leqq m<n .
$$

But $F_{0}$ is a $\$$-partition; hence

$$
\left|\sum_{n=0}^{\infty} f\left(A_{n}\right)\right|=\left|\sum_{\beta \in F_{0}} f(\beta)\right| \leqq N<\infty
$$

and $f\left(A_{n}\right)$ tends to zero as $n \rightarrow \infty$, in contradiction to the inequalities $\left|f\left(A_{n}\right)\right|>1+|f(0)|(n=1,2,3, \cdots)$.

(3.11) DEFINITION. A family $H$ is called hereditary if and only if the relation $B \subset A \in H$ implies $B \in H$.

It may be noted that the families 0 and $\{0\}$ are hereditary.

For $0<\delta \leqq \infty$ let $H_{\delta}$ represent the family of all subsets of the real number system having diameter $\left\langle\delta ; H_{\delta}\right.$ is then hereditary. If $E$ is a measurable set of real numbers and $\$$ represents the family of all measurable subsets of $E$, (B) is a $\sigma$-field and we have

$$
(5) \pi_{*} H_{\delta}=\Gamma_{\delta}(E) \text {. }
$$

(3.12) TheOREM. If \& is a $\sigma$-field, $H$ is an hereditary family, and $f$ is a function whose domain includes $\$ H$, then the relation

$$
\sup _{r \in \mathbb{B} \pi * H}\left|\sum_{\beta \in F} f(\beta)\right|=N<\infty
$$

implies

$$
\sup _{r \in \Theta^{\pi} * H} \sum_{\beta \in F}|f(\beta)|<\infty .
$$

Proof. Since sup $0=-\infty$, the theorem is trivial in the case of $B)^{*} * H=0$. Henceforth we assume (S)** $H \neq 0$; let $E=\sigma(\mathcal{B})$; let $V$ be some fixed element of $(\xi \pi * H$; for $\beta \in(B)$ let

$$
V_{\beta}=V \underset{B}{E}[B \beta \neq 0], \quad K_{\beta}=\sum_{B \in V_{\beta}}\{B \beta\} ;
$$

and let $g$ be a function with

$$
g(\beta)=\sum_{B \in v_{\beta}} f(B \beta) \quad \text { for } \beta \in \mathbb{G} .
$$

The first step in the proof is to show that the function $g$ is quasi-additive on (3. For any $F \in(B)$ let

$$
W=\sum_{\beta \in F} K_{\beta}
$$


$W$ is then an element of (5) and since $H$ is hereditary we infer $W \in(5) * H$. Moreover, it is readily seen that $K_{\beta_{1}} K_{\beta_{2}}=0$ whenever $\beta_{1} \in F, \beta_{2} \in F, \beta_{1} \neq \beta_{2}$ and also that $\left\{\beta_{1} \beta\right\}\left\{\beta_{2} \beta\right\}=0$ whenever $\beta \in F, \beta_{1} \in V_{\beta}, \beta_{2} \in V_{\beta}, \beta_{1} \neq \beta_{2}$. Two applications of Theorem (3.7) thus yield the relation

$$
\begin{aligned}
\sum_{\gamma \in W} f(\gamma) & =\sum_{\beta \in F} \sum_{\gamma \in K_{\beta}} f(\gamma)=\sum_{\beta \in F} \sum_{B \in v_{\beta}} \sum_{\gamma \in\{B \beta\}} f(\gamma) \\
& =\sum_{\beta \in F} \sum_{B \in V_{\beta}} f(B \beta)=\sum_{\beta \in F} g(\beta),
\end{aligned}
$$

an immediate implication of which is the inequality

$$
\left|\sum_{\beta \in F} g(\beta)\right| \leqq N
$$

The second step is to establish the existence of a number $M$ with

$$
\left|\sum_{\beta \in D} f(\beta)\right| \leqq M<\infty
$$

for every countable disjointed subfamily $D$ of $B H$. Using the result of the first step and Theorem (3.10) we infer the existence of a number $N_{1}$ with

$$
|g(\beta)| \leqq N_{1}<\infty \quad \text { for } \beta \in \mathbb{B} \text {. }
$$

Let $M=N+N_{1}$; let $D$ be any countable disjointed subfamily of $\$ H$; let $C=E-\sigma(D) ;$ let

$$
F_{1}=D+\sum_{B \in V_{C}}\{B C\}
$$

and note that $F_{1} \in(5) * H$. We have

$$
\begin{aligned}
\sum_{\beta \in F_{1}} f(\beta) & =\sum_{\beta \in D} f(\beta)+\sum_{B \in V_{C}} \sum_{\beta \in\{B C\}} f(\beta)=\sum_{\beta \in D} f(\beta)+\sum_{B \in V_{C}} f(B C) \\
& =\sum_{\beta \in D} f(\beta)+g(C),
\end{aligned}
$$

whence

$$
\left|\sum_{\beta \in D} f(\beta)\right| \leqq N+N_{1}=M
$$

Finally, let

$$
F \in\left(\mathcal{S}^{*} * H, \quad D_{1}=F \underset{\beta}{E}[f(\beta) \geqq 0], \quad D_{2}=F \underset{\beta}{E}[f(\beta)<0] .\right.
$$

We then have the desired conclusion:

$$
\sum_{\beta \in F}|f(\beta)|=\left|\sum_{\beta \in D_{1}} f(\beta)\right|+\left|\sum_{\beta \in D_{2}} f(\beta)\right| \leqq 2 M<\infty .
$$


It may be remarked that if in Theorem (3.12) we let $H$ stand for the hereditary family $E_{C}[C \subset \sigma(\mathbb{S})]$, then $(5 \pi * H=(5)$.

4. Concerning the behavior of the sums. The sums considered in Definition (1.5) have a considerable number of properties which are seen immediately and which it is essential to note. Partly for convenience of reference we assemble some of these properties in

(4.1) TheOREM. Let $f \in L^{*}(E)$ and $\phi$ and $\phi_{1}$ be functions each of whose domains includes $\Re(f)$.

(i) Since $0<\delta_{1} \leqq \delta_{2} \leqq \infty$ implies $\Gamma_{\delta_{1}}(E) \subset \Gamma_{\delta_{2}}(E)$, the $\inf _{F \in \Gamma_{\delta}(E)}\left[\sup _{F \in \Gamma_{\delta}(E)}\right]$ of sums of the form

$$
\sum_{B \in F} \phi\left[\mathfrak{M}_{A B} f\right]|B| \quad \text { or } \quad \sum_{B \in F}\left|\mathfrak{M}_{B} \phi: f-\phi\left[\mathfrak{M}_{A B} f\right]\right||B|
$$

is non-decreasing [non-increasing] as $\delta$ decreases $(0<\delta \leqq \infty)$.

For $0<\delta \leqq \infty$ and $F \in \Gamma_{\delta}(E)$ we have the following properties of sums:

(ii) $k=1$ implies $|A B|=|B|$ for $B \in F,|A E|=|E|$, and

$$
\sum_{B \in F} \phi\left[\mathfrak{M}_{A B} f\right]|B|=\sum_{B \in P} \phi\left[\mathfrak{M}_{B} f\right]|B| ;
$$

(iii) $0 \leqq \phi(y)$ for $y \in \Re(f)$ implies

$$
\sum_{B \in F} \phi\left[\mathfrak{M}_{A B} f\right]|B| \leqq k \sum_{B \in F} \phi\left[\mathfrak{M}_{A B} f\right]|A B|
$$

and

$$
\sup _{r \in \Gamma_{\delta}(E)} \sum_{B \in F} \phi\left[\mathfrak{M}_{A B} f\right]|B| \leqq k \sup _{r \in \Gamma_{\delta}(E)} \sum_{B \in F} \phi\left[\mathfrak{M}_{B} f\right]|B| ;
$$

(iv) $\phi(y) \leqq \phi_{1}(y)$ for $y \in \Re(f)$ implies

$$
\sum_{B \in F} \phi\left[\mathfrak{M}_{A B} f\right]|B| \leqq \sum_{B \in F} \phi_{1}\left[\mathfrak{M}_{A B} f\right]|B| ;
$$

(v) $\left(\phi+\phi_{1}\right)(y)=\phi(y)+\phi_{1}(y)$ for $y \in \Re(f)$ implies

$$
\left.\sum_{B \in F}\left(\phi+\phi_{1}\right)\left[\mathfrak{M}_{A B} f\right]|B|=\sum_{B \in F} \phi\left[\mathfrak{M}_{A B}\right]\right]|B|+\sum_{B \in F} \phi_{1}\left[\mathfrak{M}_{A B} f\right]|B|
$$

and

$$
\begin{aligned}
& \inf _{F \in \Gamma_{\delta}(E)} \sum_{B \in F} \phi\left[\mathfrak{M}_{A B}\right]|B|+\inf _{F \in \Gamma_{\delta}(E)} \sum_{B \in F} \phi_{1}\left[\mathfrak{M}_{A B} f\right]|B| \\
& \quad \leqq \inf _{F \in \Gamma_{\delta}(E)} \sum_{B \in F}\left(\phi+\phi_{1}\right)\left[\mathfrak{M}_{A B f}\right]|B| \leqq \sup _{F \in \Gamma_{\delta}(E)} \sum_{B \in F}\left(\phi+\phi_{1}\right)\left[\mathfrak{M}_{A B}\right]|B| \\
& \quad \leqq \sup _{F \in \Gamma_{\delta}(E)} \sum_{B \in F} \phi\left[\mathfrak{M}_{A B} f\right]|B|+\sup _{F \in \Gamma_{\delta}(E)} \sum_{B \in F} \phi_{1}\left[\mathfrak{M}_{A B} f\right]|B|
\end{aligned}
$$

Corresponding properties of limits of sums are:

(vi) $S_{*}(f, \phi, E, 1)=S_{*}(f, \phi, E) \leqq S^{*}(f, \phi, E)=S^{*}(f, \phi, E, 1)$; 
(vii) $0 \leqq \phi(y)$ for $y \in \Re(f)$ implies.

$$
S^{*}(f, \phi, E, k) \leqq k S^{*}(f, \phi, E) ;
$$

(viii) $\phi(y) \leqq \phi_{1}(y)$ for $y \in \Re(f)$ implies

$$
S_{*}(f, \phi, E, k) \leqq S_{*}\left(f, \phi_{1}, E, k\right), \quad S^{*}(f, \phi, E, k) \leqq S^{*}\left(f, \phi_{1}, E, k\right) ;
$$

(ix) $\left(\phi+\phi_{1}\right)(y)=\phi(y)+\phi_{1}(y)$ for $y \in \Re(f)$ implies

$$
\begin{aligned}
S_{*}(f, \phi, E, k)+S_{*}\left(f, \phi_{1}, E, k\right) \leqq S_{*}\left(f, \phi+\phi_{1}, E, k\right) & \\
& \leqq S^{*}\left(f, \phi+\phi_{1}, E, k\right) \leqq S^{*}(f, \phi, E, k)+S^{*}\left(f, \phi_{1}, E, k\right) .
\end{aligned}
$$

It must be emphasized that when we write, as in the theorem above,

$$
\sum_{B \in F} \phi\left[\mathfrak{M}_{A B}\right]|| B \mid,
$$

without inf or sup ahead of it, $A$ is understood to be an arbitrary fixed set satisfying condition (1.6); whereas, when

$$
\inf _{F \in \Gamma_{\delta}(E)} \text { or } \sup _{r \in \Gamma_{\delta}(E)} \sum_{B \in F} \phi\left[\mathfrak{M}_{A B}\right]|B|
$$

is written, $A$ is understood to vary with $F$ with the freedom allowed by condition (1.6). To define a notation that makes this distinction would introduce, it seems to us, an unnecessary complication.

The next four theorems are fundamental for later developments.

(4.2) Theorem. If $f \in L^{*}(E)$, $\phi$ is a function whose domain includes $\Re(f)$, and $0<\delta \leqq \infty$, the condition

implies

$$
\sup _{F \in \Gamma_{\delta}(B)}\left|\sum_{B \in F} \phi\left[\mathfrak{M}_{B} f\right]\right| B||<\infty
$$

$$
\sup _{r \in \Gamma_{\delta}(E)} \sum_{B \in F}\left|\phi\left[\mathfrak{M}_{B} f\right]\right||B|<\infty .
$$

Taking into account the remark immediately preceding the statement of Theorem (3.12), one sees at once that this theorem is a corollary of Theorem (3.12).

Corollary. If. $f \in L^{*}(E)$ and $\phi$ is a function whose domain includes $\Re(f)$, the condition

$$
-\infty<S_{*}(f, \phi, E) \leqq S^{*}(f, \phi, E)<\infty
$$

implies $0 \leqq S^{*}(f,|\phi|, E)<\infty$.

(4.3) Theorem. If $f \in L^{*}(E), 0 \leqq \phi(y)$ for $y \in \Re(f)$, and $0<\delta \leqq \infty$, the condition 


$$
\sup _{r \in \Gamma_{\delta}(E)} \sum_{B \in F} \phi\left[\mathfrak{M}_{B} f\right]|B|=M<\infty
$$

implies the existence of $N=N(\epsilon)>0$ with

$$
0<N<\infty, \quad \sup _{r \in \Gamma_{\delta}(H)} \sum_{B \in F} \phi\left[\mathfrak{M}_{B} f\right]|B|<\epsilon \text { for } \quad H \subset E-[-N, N] \text {. }
$$

Proof. Let $F_{1} \in \Gamma_{\delta}(E)$ be such that

$$
\sum_{B \in F_{1}} \phi\left[\mathfrak{M}_{B} f\right]|B|>M-\epsilon .
$$

Clearly $F_{1}$ contains a finite family $F_{2}$ satisfying the same inequality. Let $N$ satisfy the conditions

$$
0<N<\infty, \quad \sigma\left(F_{2}\right) \subset[-N, N] .
$$

For $H \subset E-[-N, N]$ and $F \in \Gamma_{\delta}(H)$, we then have the desired condition.

(4.4) Theorem. If $f \in L^{*}(E), \phi \in C(\Re(f)), 0 \leqq \phi(y)$ for $y \in \Re(f), 0<\delta \leqq \infty$,

$$
\sup _{r \in \Gamma_{\delta}(H)} \sum_{B \in F} \phi\left[\mathfrak{M}_{B} f\right]|B|<\infty,
$$

and $H$ is a measurable subset of $E$, then we have

$$
\lim _{|H| \rightarrow 0} \sup _{F \in \Gamma_{\delta}(H)} \sum_{B \in F} \phi\left[\mathfrak{M}_{B} f\right]|B|=0 .
$$

Proof. Let

$$
\begin{aligned}
\Lambda(B) & =\phi\left[\mathfrak{M}_{B} f\right]|B| & \text { for } B \in F \in \Gamma_{\delta}(H), \\
M & =\limsup _{|H| \rightarrow 0} \sum_{B \in F \in \Gamma_{\delta}(H)} \Lambda(B), &
\end{aligned}
$$

$M$ being finite on account of the hypotheses. If $M$ is $>0$, there exists $\eta>0$ such that $|\dot{H}|<\eta$ implies

$$
\sum_{B \in F \in \Gamma_{\delta}(H)} \Lambda(B) \leqq 3 M / 2
$$

Let $H_{1}$ be a particular set $H$ and $F_{1} \in \Gamma_{\delta}\left(H_{1}\right)$ a particular family of sets satisfying the conditions

$$
\left|H_{1}\right|<\eta / 2, \quad \sum_{B \in F_{1}} \Lambda(B)>4 M / 5 .
$$

It is clear that $F_{1}$ can be taken as a finite family. If $D$ is a measurable subset of $E$, we have

$$
\lim _{|D| \rightarrow 0} \int_{B \rightarrow D} f /|B-D|=\int_{B} f /|B| \quad \text { for } B \in F_{1},|B|>0 ;
$$

hence, in view of the continuity of $\phi$ on $\Re(f)$ and the finiteness of the family 
$F_{1}$, there exists $\gamma$ with $0<\gamma<\eta / 2$ such that $|D|<\gamma$ implies

$$
\sum_{B \in F_{1}} \Lambda(B-D)>4 M / 5 \text {. }
$$

Let $H_{2}$ be a particular set $H$ and $F_{2} \in \Gamma_{\delta}\left(H_{2}\right)$ a particular family of sets satisfying the conditions

Let

$$
\left|H_{2}\right|<\gamma, \quad \sum_{B \in F_{2}} \Lambda(B)>4 M / 5
$$

$$
G=\sum_{B \in F_{1}}\left\{B-H_{2}\right\}
$$

and consider the family $G+F_{2}$ of disjoint sets. We have

$$
\sum_{B \in G+F_{2}}|B| \leqq \sum_{B \in F_{1}}|B|+\sum_{B \in F_{2}}|B|=\left|H_{1}\right|+\left|H_{2}\right|<\eta / 2+\gamma<\eta,
$$

whence

$$
3 M / 2 \geqq \sum_{B \in G+F_{2}} \Lambda(B)=\sum_{B \in G} \Lambda(B)+\sum_{B \in F_{2}} \Lambda(B)>4 M / 5+4 M / 5,
$$

a contradiction which implies $M=0$ and completes the proof.

Combining Theorems (4.2) and (4.4) we obtain the following result.

(4.5) ThEOREM. If $f \in L^{*}(E), \phi \in C(\Re(f))$, and $H$ is a measurable subset of $E$, the condition

$$
-\infty<S_{*}(f, \phi, E) \leqq S^{*}(f, \phi, E)<\infty
$$

implies

$$
\lim _{|H| \rightarrow 0} S^{*}(f,|\phi|, H)=0 .
$$

In other words, the conclusion of this theorem is that $S^{*}(f,|\phi|, H)$ is an absolutely continuous function of a measurable set $H \subset E$.

5. Sufficient conditions for (1.9). Since (1.9) is trivially true when $|E|=0$, we are justified in assuming $|E|>0$ in connection with the proof of each theorem in this section. The first theorem is an extension of the lemma of RS

(5.1) Theorem. If $E$ is essentially bounded, the conditions $f \in U C(E)$ and $\phi \in C(\overline{\Re(f)})$ are sufficient for (1.9), even with (1.6) replaced by (2.10).

Proof. We may assume $f$ itself to be uniformly continuous on $E$; then $f$ is bounded on $E$, and $\overline{\Re(f)}$ is seen to be a bounded closed interval. Thus $\phi \in C(\overline{\Re(f)})$ implies $\phi \in U C(\overline{\Re(f)}) ; \phi: f$ is uniformly continuous on $E$; and we have

$$
\int_{E} \phi: f=\sum_{B \in F} \int_{B} \phi: f \quad \text { for } F \in \Gamma_{\delta}(E), 0<\delta \leqq \infty .
$$


Let $\eta=\eta(\phi, \epsilon)>0$ be such that

$$
y_{1}, y_{2} \in \overline{\Re(f)},\left|y_{1}-y_{2}\right| \leqq \eta \text { imply }\left|\phi\left(y_{1}\right)-\phi\left(y_{2}\right)\right| \leqq \epsilon /(2|E|) ;
$$

and let $\delta=\delta(f, \eta)>0$ be such that

$$
x_{1}, x_{2} \in E,\left|x_{1}-x_{2}\right| \leqq \delta \text { imply }\left|f\left(x_{1}\right)-f\left(x_{2}\right)\right| \leqq \eta .
$$

For $F \in \Gamma_{\delta}(E), B \in F,|B|>0$, and $x_{1} \in B$ we then have

$$
\begin{aligned}
& \alpha=\inf _{x \in B} \phi[f(x)] \leqq \mathfrak{M}_{B} \phi: f, \phi\left[f\left(x_{1}\right)\right] \leqq \sup _{x \in B} \phi[f(x)]=\beta, \beta-\alpha \leqq \epsilon /(2|E|), \\
& a=\inf _{x \in B} f(x) \leqq \mathfrak{M}_{A B} f, \quad f\left(x_{1}\right) \leqq \sup _{x \in B} f(x)=b, \quad b-a \leqq \eta,
\end{aligned}
$$

whence

and

$$
\begin{aligned}
\left|\mathfrak{M}_{B} \phi: f-\phi\left[\mathfrak{M}_{A B} f\right]\right| \leqq & \left|\mathfrak{M}_{B} \phi: f-\phi\left[f\left(x_{1}\right)\right]\right| \\
& +\left|\phi\left[f\left(x_{1}\right)\right]-\phi\left[\mathfrak{M}_{A B} f\right]\right| \leqq \epsilon /|E|
\end{aligned}
$$

$$
\sum_{B \in F}\left|\mathfrak{M}_{B} \phi: f-\phi\left[\mathfrak{M}_{A B} f\right]\right||B| \leqq \epsilon .
$$

REMARK. The relation (1.9) can be established by means of inequality (a) on the individual terms of (1.9) only under very special circumstances, such as those hypothesized in Theorem (5.1). That this cannot be done even in the simple case of $E=I, \phi(y)=y$ for $y \in \overline{\Re(f)}$, when $k$ is $>1$ and $f$ has a single point of essential discontinuity in $I$ is illustrated by taking $f$ on $I$ to be the characteristic function of the set $E_{x}[1 / 2 \leqq x \leqq 1]$.

(5.2) THEOREM. If $E$ is essentially bounded, the conditions $f \in L^{*}(E)=L(E)$ and $\phi \in U C(\Re(f))$ are sufficient for (1.9).

Proof. If the interval $\Re(f)$ is not a closed set, it has a finite endpoint which does not belong to it. The hypothesis $\phi \in U C(\Re(f))$ implies that $\lim \phi(y)$ exists as $y \in \Re(f)$ approaches this endpoint. If at each such endpoint $\phi$ is defined (or redefined) as this limit, we have $\phi \in U C(\overline{\Re(f)})$. Clearly this extension of the definition of $\phi$ (or this redefinition of $\phi$ ) does not affect the sums in question; neither does it affect the value of $\int_{E} \phi: f$, since the set of points $E E_{x}[f(x) \tilde{\varepsilon} \Re(f)]$ is of measure zero. In other words, we may assume $\phi \in U C(\overline{\Re(f)})$.

Let $0<\epsilon_{1}<\epsilon /(1+|E|)$ and $\eta=\eta\left(\phi, \epsilon_{1}\right)>0$ be such that

$$
y_{1}, y_{2} \in \overline{\Re(f)},\left|y_{1}-y_{2}\right| \leqq \eta \text { imply }\left|\phi(y)_{1}-\phi\left(y_{2}\right)\right| \leqq \epsilon_{1} / 4 ;
$$

then we have

$$
\left|\phi\left(y_{1}\right)-\phi\left(y_{2}\right)\right| \leqq \epsilon_{1} / 4+\epsilon_{1}\left|y_{1}-y_{2}\right| /(4 \eta) \text { for } \quad y_{1}, y_{2} \in \overline{\Re(f)} .
$$

Let $K=[a, b]$ be a bounded closed interval containing all of $E$ except for 
a set of measure zero which can and will be neglected; let $f$ be a particular function summable on $E$; and let $x_{1}$ satisfy the conditions

$$
x_{1} \in E, \quad \underset{x \in E}{\operatorname{ess} \inf } f(x) \leqq f\left(x_{1}\right) \leqq \underset{x \in E}{\operatorname{ess} \sup } f(x), \quad-\infty<f\left(x_{1}\right)<\infty .
$$

Then $f$ can be defined (or redefined) as $f\left(x_{1}\right)$ for $x \in K-E$, so that $f$ is summable on $[a, b]$ and the new $\Re(f)$ is the same as the old $\Re(f)$. On $K$ the function $f$ can be approximated arbitrarily closely in the mean by a function $g$ continuous on $K$ and having the property

$$
\underset{x \in K}{\text { ess inf }} f(x) \leqq \underset{x \in K}{\operatorname{ess} \inf } g(x) \leqq \underset{x \in K}{\operatorname{ess} \sup } g(x) \leqq \underset{x \in K}{\operatorname{ess} \sup } f(x) .
$$

Thus we are assured of the existence of a function $g$ satisfying the conditions

$$
g \in U C(E), \quad \int_{E}|f-g|<\eta / k \leqq \eta, \quad \overline{\Re(g)} \subset \overline{\Re(f)} .
$$

In accordance with Theorem (5.1) let $\delta=\delta\left(g, \phi, \epsilon_{1} / 4\right)>0$ correspond to $\epsilon_{1} / 4$ for the functions $g$ and $\phi$. The function $\phi: f$ is measurable on $E$ and we infer that it is summable on $E$ from the inequality

$$
|\phi[f(x)]| \leqq|\phi[g(x)]|+\epsilon_{1} / 4+\epsilon_{1}|f(x)-g(x)| /(4 \eta) \quad \text { for } x \in E,
$$

which expresses the dominance of $|\phi: f|$ by a summable function. For $F \in \Gamma_{\delta}(E)$ we now have

$$
\begin{aligned}
& \sum_{B \in F}\left|\int_{B} \phi: f-\phi\left[\mathfrak{M}_{A B} f\right]\right| B|| \\
& \leqq \sum_{B \in F}\left(\left|\int_{B} \phi: f-\int_{B} \phi: g\right|+\left|\int_{B} \phi: g-\phi\left[\mathfrak{M}_{A B} g\right]\right| B||\right. \\
& \left.\quad+\left|\phi\left[\mathfrak{M}_{A B} g\right]-\phi\left[\mathfrak{M}_{A B} f\right]\right||B|\right) \\
& \leqq \int_{E}|\phi: f-\phi: g|+\epsilon_{1} / 4+\sum_{B \in F}\left(\epsilon_{1} / 4+\epsilon_{1} \int_{A B}|g-f| /(4 \eta|A B|)\right)|B| \\
& \leqq \epsilon_{1} / 4+\epsilon_{1} \int_{E}|f-g| /(4 \eta)+\epsilon_{1} / 4+\left(\epsilon_{1} / 4\right)|E| \\
& \quad+k \epsilon_{1} \int_{E}|g-f| /(4 \eta)<\epsilon_{1}+\epsilon_{1}|E|<\epsilon .
\end{aligned}
$$

For use in proving subsequent theorems we introduce two definitions and a lemma.

(5.3) Definition. If $f \in L^{*}(E), \sum_{i=1}^{\infty} J_{i}$ will be called a normal expression for $\Re(f)$ if and only if the following conditions are satisfied: (1) for each $i, J_{i}$ is a 
bounded closed interval $\left[a_{i}, b_{i}\right] \subset \Re(f)$, with

$$
\left[a_{i}, b_{i}\right] \subset J_{i} \subset J_{i+1} \quad(i=1,2,3, \cdots) ;
$$

(2) if inf $\Re(f)=a$ is finite and $a \in \Re(f), a \in J_{i}(i=1,2,3, \ldots)$; (3) if sup $\Re(f)=b$ is finite and $b \in \Re(f), b \in J_{i}(i=1,2,3, \cdots)$; and (4) $\sum_{i=1}^{\infty} J_{i}$ $=\Re(f)$.

It should be observed that if $\Re(f)$ is a bounded closed interval, $J_{i}=\Re(f)$ for each $i$.

(5.4) Lemma. Let $f \in L(E)$ and $\sum_{i=1}^{\infty} J_{i}$ be a normal expression for $\Re(f)$. For $0<\delta \leqq \infty$ and $F \in \Gamma_{\delta}(E)$ let $G_{i} \subset F$ represent the family of sets $B \in F$ defined by the condition

$$
\mathfrak{M}_{B} f \in \Re(f)-J_{i} \quad(i=1,2,3, \cdots) .
$$

Then we have

$$
\lim _{i \rightarrow \infty} \sup _{F \in \Gamma_{\delta}(E)} \sum_{B \in G_{i}}|B|=0 .
$$

Proof. In case $\Re(f)$ is a bounded closed interval, $\Re(f)-J_{i}$ is vacuous and $\sum_{B \in G_{i}}|B|=0$ for each $i$. In case sup $\Re(f)=\infty$ we have $\lim _{i \rightarrow \infty} b_{i}=\infty$. For each $i$ such that $b_{i}>0$, let $H_{i} \subset F$ represent the family of sets $B$ defined by the condition $\mathfrak{M}_{B} f>b_{i}$. Then we have

$$
\sum_{B \in H_{i}}|B|=\sum_{B \in H_{i}} \int_{B} f|B| / \int_{B} f=\sum_{B \in H_{i}} \int_{B} f / \mathfrak{M}_{B} f<\left(1 / b_{i}\right) \int_{E}|f|,
$$

which tends to zero with $i$.

In case sup $\Re(f)=b<\infty, b \tilde{\varepsilon} \Re(f)$, we have $\lim _{i \rightarrow \infty} b_{i}=b$. Let $H_{i} \subset F$ stand for the family of sets $B$ defined by the condition $\mathfrak{M}_{B} f>b_{i}$, and let $\epsilon_{i}=b-b_{i}$ $(i=1,2,3, \cdots)$. For any particular $i$ and $B \in H_{i}$ let

$$
\begin{gathered}
B_{1}=B \underset{x}{E}\left[b-\epsilon_{i}<f(x)\right], \quad B_{2}=B \underset{x}{E}\left[f(x)<b-2 \epsilon_{i}\right], \\
B_{3}=B \underset{x}{E}\left[b-2 \epsilon_{i} \leqq f(x) \leqq b-\epsilon_{i}\right],
\end{gathered}
$$

so that we have

$$
\begin{aligned}
b-\epsilon_{i}<\mathfrak{M}_{B} f & =\frac{\left|B_{1}\right|}{|B|} \mathfrak{M}_{B_{1}} f+\frac{\left|B_{2}\right|}{|B|} \mathfrak{M}_{B_{2}} f+\frac{\left|B_{3}\right|}{|B|} \mathfrak{M}_{B_{3} f} \\
& <\frac{\left|B_{1}\right|}{|B|} b+\frac{\left|B_{2}\right|}{|B|}\left(b-2 \epsilon_{i}\right)+\frac{\left|B_{3}\right|}{|B|}\left(b-\epsilon_{i}\right) \\
& =b-\epsilon_{i}\left(2\left|B_{2}\right| /|B|+\left|B_{3}\right| /|B|\right)
\end{aligned}
$$

whence, since $\epsilon_{i}$ is $>0$, 
and

$$
\begin{gathered}
2\left|B_{2}\right| /|B|+\left|B_{3}\right| /|B|<1, \\
2\left|B_{2}\right|+\left|B_{3}\right|<|B|=\left|B_{1}\right|+\left|B_{2}\right|+\left|B_{3}\right|, \\
\left|B_{2}\right|<\left|B_{1}\right|
\end{gathered}
$$

$$
|B|<2\left|B_{1}\right|+\left|B_{3}\right| \leqq 2\left(\left|B_{1}\right|+\left|B_{3}\right|\right) \text {. }
$$

Hence we obtain

$$
\sum_{B \in H_{i}}|B| \leqq 2\left|\underset{x}{E}\left[b-2 \epsilon_{i} \leqq f(x)\right]\right|
$$

But from the inequality $\epsilon_{i+1} \leqq \epsilon_{i}(i=1,2,3, \cdots)$ we have

$$
\underset{x}{E}\left[b-2 \epsilon_{i+1} \leqq f(x)\right] \subset \underset{x}{E}\left[b-2 \epsilon_{i} \leqq f(x)\right] \quad(i=1,2,3, \cdots)
$$

which implies

$$
\begin{aligned}
0 & \leqq \lim _{i \rightarrow \infty} \sup _{F \in \mathrm{r}_{\delta}(E)} \sum_{B \in H_{i}}|B| \leqq 2 \lim _{i \rightarrow \infty}\left|\underset{x}{E}\left[b-2 \epsilon_{i} \leqq f(x)\right]\right| \\
& =2\left|\prod_{i=1}^{\infty} \underset{x}{E}\left[b-2 \epsilon_{i} \leqq f(x)\right]\right|=2|\underset{x}{E}[b \leqq f(x)]|=0 .
\end{aligned}
$$

The cases inf $\Re(f)=-\infty$ and inf $\Re(f)=a>-\infty, a \tilde{\varepsilon} \Re(f)$ are entirely similar to those already considered, so that the lemma may now be regarded as established.

(5.5) Definition. For $f \in L^{*}(E), \phi \in C(\Re(f)), 0 \leqq \phi(y)$ when $y \in \Re(f)$, and $\sum_{i=1}^{\infty} J_{i}$ a normal expression for $\Re(f)$, we call the sequence $\phi_{i}(i=1,2,3, \cdots)$, where for each $i$

$$
\phi_{i}(y)=\left\{\begin{array}{lll}
\phi(y) & \text { for } & y \in J_{i}=\left[a_{i}, b_{i}\right], \\
\inf _{y \leqq t \leqq a_{i}} \phi(t) & \text { for } & a_{i}>y \in \Re(f), \\
\inf _{b_{i} \leqq t \leqq y} \phi(t) & \text { for } & b_{i}<y \in \Re(f),
\end{array}\right.
$$

a normal approximating sequence for $\phi$ on $\Re(f)$.

We note that if $\Re(f)$ is a bounded closed interval, $\phi_{i}$ is identical with $\phi$ for each $i$. It is essential to observe also the following properties of a normal approximating sequence:

$$
\begin{aligned}
& \phi_{i} \in U C(\Re(f)), \\
& \phi(y)-\phi_{i}(y)=0 \text { for } y \in J_{i}, \\
& 0 \leqq \phi_{i}(y) \leqq \phi(y) \text { for } y \in \Re(f), \quad(i=1,2,3, \cdots) ; \\
& \lim _{i \rightarrow \infty} \phi_{i}(y)=\phi(y) \text { for } y \in \Re(f) .
\end{aligned}
$$


Since the set of points $E E_{x}[f(x) \tilde{\varepsilon} \Re(f)]$ is of measure zero, it follows that - we have

$$
\left.\begin{array}{l}
0 \leqq \phi_{i}[f(x)] \leqq \phi[f(x)] \quad(i=1,2,3, \cdots) \\
\lim _{i \rightarrow \infty} \phi_{i}[(f x)]=\phi[f(x)]
\end{array}\right\} \quad \text { for almost all } x \in E
$$

(5.7) Theorem. The conditions $f \in L^{*}(E)$ and $\phi \in C(\Re(f)$ ) imply

$$
\left|\int_{E} \phi: f\right| \leqq \int_{E}|\phi: f| \leqq S_{*}(f,|\phi|, E) \leqq \infty .
$$

Proof. Let $\phi_{i}(i=1,2,3, \cdots)$ be a normal approximating sequence for $|\phi|$ on $\Re(f)$, and let $H$ represent any essentially bounded measurable subset of $E$. Theorems (5.2) and (4.1) (viii) imply

$$
\begin{array}{r}
\int_{H} \phi_{i}: f=S\left(f, \phi_{i}, H\right)=S_{*}\left(f, \phi_{i}, H\right) \leqq S_{*}(f,|\phi|, H) \leqq S_{*}(f,|\phi|, E) \leqq \infty \\
(i=1,2,3, \cdots) ;
\end{array}
$$

and by aid of (5.6) and a well known theorem of Fatou we obtain

whence

$$
\int_{H}|\phi: f| \leqq \liminf _{i \rightarrow \infty} \int_{H} \phi_{i}: f
$$

$$
\int_{H}|\phi: f| \leqq S_{*}(f,|\phi|, E) .
$$

The desired conclusion then follows from the arbitrariness of $H$.

(5.8) THEOREM. The conditions $f \in L^{*}(E), \phi \in U C(\Re(f)), 0 \leqq \phi(y)$ for $y \in \Re(f)$, and $S^{*}(f, \phi, E)<\infty$ are sufficient for (1.9).

Proof. If $E$ is essentially bounded, the conclusion follows from Theorem (5.2) without use of the hypothesis $S^{*}(f, \phi, E)<\infty$. Otherwise, this hypothesis implies the existence of $\eta$ satisfying the conditions

$$
0<\eta<\infty, \quad \sup _{F \in \Gamma_{\eta}(E)} \sum_{B \in F} \phi\left[\mathfrak{M}_{B} f\right]|B|<\infty .
$$

In accordance with Theorem (4.3) let $N_{1}=N_{1}(\epsilon / \overline{3 k})>0$ insure

$$
\sup _{F \in \Gamma_{\eta}(H)} \sum_{B \in F} \phi\left[\mathfrak{M}_{B} f\right]|B|<\epsilon /(3 k) \quad \text { for } H \subset E-\left[-N_{1}, N_{1}\right] .
$$

From Theorem (5.7) we infer $\int_{E} \phi: f<\infty$; thus there exists $N$ with

$$
N_{1} \leqq N<\infty, \quad \int_{H} \phi: f<\epsilon / 3 \quad \text { for } H \subset E-[-N, N]
$$


Let

$$
E_{N}=E \underset{x}{E}[-N-\eta \leqq x \leqq N+\eta]
$$

and in accordance with Theorem (5.2) let $\delta=\delta\left(f, \phi, E_{N}, \epsilon / 3\right), 0<\delta \leqq \eta$, correspond to $\epsilon / 3$ for the functions $f$ and $\phi$ on $E_{N}$. For $F \in \Gamma_{\delta}(E)$ let $F_{1} \subset F$ stand for the family of sets $B \in F$ defined by the condition $B \subset E_{N}$, and let $F_{2}=F-F_{1}$. Then we have, by aid of Theorem (4.1) (iii),

$$
\begin{aligned}
\sum_{B \in F}\left|\int_{B} \phi: f-\phi\left[\mathfrak{M}_{A B} f\right]\right| B|| \\
\quad \leqq \sum_{B \in F_{1}}\left|\int_{B} \phi: f-\phi\left[\mathfrak{M}_{A B} f\right]\right| B||+\sum_{B \in F_{2}} \int_{B} \phi: f+\sum_{B \in F_{2}} \phi\left[\mathfrak{M}_{A B} f\right]|B| \\
<\epsilon / 3+\epsilon / 3+k \sup _{F \in \Gamma_{\delta}(H)} \phi\left[\mathfrak{M}_{B} f\right]|B|<\epsilon,
\end{aligned}
$$

where $H=\sigma\left(F_{2}\right) \subset E-\left[-N_{1}, N_{1}\right]$.

For the proof of the next theorem the following lemma will prove helpful.

(5.10) Lemma. Let $f \in L^{*}(E), \phi \in C(\Re(f)), 0 \leqq \phi(y)$ for $y \in \Re(f), S^{*}(f, \phi, E)$ $<\infty$, and $\phi_{i}(i=1,2,3, \cdots)$ be a normal approximating sequence for $\phi$ on $\Re(f)$. Then there exists $\eta$ with $0<\eta<\infty$ such that we have

$$
\lim _{i \rightarrow \infty} \sup _{r \in \Gamma_{\eta}(E)} \sum_{B \in F}\left(\phi-\phi_{i}\right)\left[\mathfrak{M}_{A B} f\right]|B|=0 .
$$

Proof. If $\Re(f)$ is a bounded closed interval, $\phi(y)-\phi_{i}(y)=0$ for $y \in \Re(f)$ $(i=1,2,3, \cdots)$ and the conclusion is obvious with any $\eta$ without use of the hypothesis $S^{*}(f, \phi, E)<\infty$. Otherwise, this hypothesis implies the existence of $\eta$ satisfying conditions (5.9). In accordance with Theorem (4.3) let $N=N(\epsilon / \overline{2 k})>\eta>0$ be such that

$$
\sup _{R \in \Gamma_{\eta}(H)} \sum_{B \in F} \phi\left[\mathfrak{M}_{B} f\right]|B|<\epsilon /(2 k) \quad \text { for } H \subset E-[-N, N],
$$

and in accordance with Theorem (4.4) let $\gamma=\gamma(f, \phi, \eta, \epsilon /(2 k))>0$ be such that

$$
H \subset E, \quad|H|<\gamma \text { imply } \sup _{\boldsymbol{F} \in \Gamma_{\eta}(H)} \sum_{B \in F} \phi\left[\mathfrak{M}_{B} f\right]|B|<\epsilon /(2 k) .
$$

Let $E_{N}=[-N-\eta, N+\eta] E$ and in accordance with Lemma (5.4) let $m$ be such that

$$
\sup _{r \in \Gamma_{\eta}\left(E_{N}\right)} \sum_{B \in G_{i}}|B|<\gamma \quad \text { for } i>m,
$$

where $G_{i} \subset F$ has the meaning ascribed to it in that lemma. For $F \in \Gamma_{\eta}(E)$ let $F_{1} \subset F$ be defined by the condition $B \in F_{1}$ if and only if $[-N, N] B \neq 0$, and let $F_{2}=F-F_{1}$. For each $i$ we then have, by aid of Theorem (4.1) (iii), 


$$
\begin{aligned}
\sup _{r \in \mathrm{r}_{\eta}(E)} & \sum_{B \in F}\left(\phi-\phi_{i}\right)\left[\mathfrak{M}_{A B} f\right]|B| \leqq k \sup _{F \in \Gamma_{\eta}(B)} \sum_{B \in F}\left(\phi-\phi_{i}\right)\left[\mathfrak{M}_{B} f\right]|B| \\
& \leqq k \sup _{F_{1}} \sum_{B \in F_{1}}\left(\phi-\phi_{i}\right)\left[\mathfrak{M}_{B} f\right]|B|+k \sup _{F_{2}} \sum_{B \in F_{2}}\left(\phi-\phi_{i}\right)\left[\mathfrak{M}_{B} f\right]|B|,
\end{aligned}
$$

where the second term does not exceed

$$
k \sup _{F_{2}} \sum_{B \in F_{2}} \phi\left[\mathfrak{M}_{B} f\right]|B|<k \epsilon /(2 k)=\epsilon / 2
$$

and for $i>m$ the first term is less than or equal to

$$
\begin{array}{r}
k \sup _{F \in \Gamma_{\eta}\left(E_{N}\right)} \sum_{B \in F}\left(\phi-\phi_{i}\right)\left[\mathfrak{M}_{B} f\right]|B|=k \sup _{F \in \Gamma_{\eta}\left(E_{N}\right)} \sum_{B \in G_{i}}\left(\phi-\phi_{i}\right)\left[\mathfrak{M}_{B} f\right]|B| \\
\leqq k \sup _{r \in \Gamma_{\eta}\left(E_{N}\right)} \sum_{B \in G_{i}} \phi\left[\mathfrak{M}_{B} f\right]|B|<k \epsilon /(2 k)=\epsilon / 2 .
\end{array}
$$

COROLlary. The hypotheses of this lemma imply

$$
\lim _{i \rightarrow \infty} S^{*}\left(f, \phi-\phi_{i}, E, k\right)=0 .
$$

(5.11) ThEOREM. If $f \in L^{*}(E), \phi \in C(\Re(f))$, and $0 \leqq \phi(y)$ for $y \in \Re(f)$, the condition $S^{*}(f, \phi, E)<\infty$ is sufficient (as well as obviously necessary) for (1.9).

Proof. If $\Re(f)$ is a bounded closed interval, the conclusion follows at once from Theorem (5.8). Otherwise, the hypothesis $S^{*}(f, \phi, E)<\infty$, by Theorem (5.7), implies the existence of $\int_{E} \phi: f$; and it also implies the existence of $\eta$ satisfying conditions (5.9). Let $\phi_{i}(i=1,2,3, \cdots)$ be a normal approximating sequence for $\phi$ on $\Re(f)$. Then for $0<\delta \leqq \eta, F \in \Gamma_{\delta}(E)$, and each $i$ we may write

$$
\begin{aligned}
& \sum_{B \in F}\left|\int_{B} \phi: f-\phi\left[\mathfrak{M}_{A B} f\right]\right| B|| \\
& \leqq \int_{E}\left|\phi: f-\phi_{i}: f\right|+\sum_{B \in F}\left|\int_{B} \phi_{i}: f-\phi_{i}\left[\mathfrak{M}_{A B} f\right]\right| B|| \\
&+\sum_{B \in F}\left|\phi_{i}\left[\mathfrak{M}_{A B} f\right]-\phi\left[\mathfrak{M}_{A B} f\right]\right||B|
\end{aligned}
$$

From (5.6) and the inequality $\int_{E} \phi: f<\infty$ we infer, by Lebesgue's convergence theorem, the existence of a number $N_{1}$ with

$$
\int_{E}\left|\phi: f-\phi_{i}: f\right|<\epsilon / 3 \quad \text { for } i>N_{1} \text {. }
$$

Lemma (5.10) implies the existence of a number $N \geqq N_{1}$ with

$$
\begin{aligned}
\sup _{F \in \Gamma_{\delta}(E)} & \sum_{B \in F}\left|\phi_{i}\left[\mathfrak{M}_{A B} f\right]-\phi\left[\mathfrak{M}_{A B} f\right]\right||B| \\
& =\sup _{F \in \Gamma_{\delta}(E)} \sum_{B \in F}\left(\phi-\phi_{i}\right)\left[\mathfrak{M}_{A B} f\right]|B|
\end{aligned}
$$




$$
\leqq \sup _{r \in \Gamma_{\eta}(E)} \sum_{B \in F}\left(\phi-\phi_{i}\right)\left[\mathfrak{M}_{A B} f\right]|B|<\epsilon / 3 \text { for } i>N .
$$

Having fixed $i>N$, in accordance with Theorem (5.8) we let $\delta=\delta\left(f, \phi_{i}, \epsilon / 3\right)$, $0<\delta \leqq \eta$, be such that the second term on the right in (a) is $<\epsilon / 3$. This completes the proof.

(5.12) THEOREM. If $f \in L^{*}(E)$ and $\phi \in C(\Re(f))$, the condition

$$
-\infty<S_{*}(f, \phi, E) \leqq S^{*}(f, \phi, E)<\infty
$$

is sufficient (as well as obviously necessary) for (1.9).

Proof. By the corollary to Theorem (4.2), the hypotheses of the present theorem imply $S^{*}(f,|\phi|, E)<\infty$. For $y \in \Re(f)$ let

$$
\phi_{1}(y)=\left[|\phi(y)|+\phi^{\prime}(y)\right] / 2, \quad \phi_{2}(y)=[|\phi(y)|-\phi(y)] / 2,
$$

whence

$$
\phi(y)=\phi_{1}(y)-\phi_{2}(y)
$$

and for $i=1,2$ we have

$\phi_{i} \in C(\Re(f)), 0 \leqq \phi_{i}(y) \leqq|\phi(y)|$ for $y \in \Re(f), S^{*}\left(f, \phi_{i}, E\right) \leqq S^{*}(f,|\phi|, E)<\infty$.

In accordance with Theorem (5.11), therefore, let $\delta_{i}=\delta_{i}\left(f, \phi_{i}, \epsilon / 2\right)>0$ correspond to $\epsilon / 2$ for the functions $f$ and $\phi_{i}(i=1,2)$. Then we have, for $\delta=\min \left[\delta_{1}, \delta_{2}\right]$ and $F \in \Gamma_{\delta}(E)$,

$$
\begin{aligned}
\sum_{B \in F} \mid & \int_{B} \phi: f-\phi\left[\mathfrak{M}_{A B} f\right]|B| \mid \\
\quad & =\sum_{B \in F}\left|\int_{B} \phi_{1}: f-\int_{B} \phi_{2}: f-\phi_{1}\left[\mathfrak{M}_{A B} f\right]\right| B\left|+\phi_{2}\left[\mathfrak{M}_{A B} f\right]\right| B|| \\
& \leqq \sum_{B \in F}\left|\int_{B} \phi_{1}: f-\phi_{1}\left[\mathfrak{M}_{A B} f\right]\right| B||+\sum_{B \in F}\left|\int_{B} \phi_{2}: f-\phi_{2}\left[\mathfrak{M}_{A B} f\right]\right| B||<\epsilon .
\end{aligned}
$$

The following corollary is now evident.

Corollary. For $f \in L^{*}(E)$ and $\phi \in C(\Re(f))$, the conditions (1.8), (1.9), (1.10), and (1.11) are equivalent.

(5.13) THEOREM. If $f \in L^{*}(E)$ and $\phi \in C(\Re(f))$, the conditions $|\phi(y)| \leqq \psi(y)$ for $y \in \Re(f), \psi$ convex on $\Re(f)$, and $\int_{E} \psi: f<\infty$ are sufficient for

$$
\sup _{r \in \Gamma_{\eta}(E)}\left|\sum_{B \in F} \phi\left[\mathfrak{M}_{B} f\right]\right| B||<\infty, \quad \eta=\operatorname{diam} E,
$$

and therefore for (1.9). 
Proof. Using Jensen's inequality $\left(^{(}\right)$we obtain, for $F \in \Gamma_{\mathfrak{y}}(E)$,

$$
\begin{aligned}
\left|\sum_{B \in F} \phi\left[\mathfrak{M}_{B} f\right]\right| B|| & \leqq \sum_{B \in F}\left|\phi\left[\mathfrak{M}_{B} f\right]\right||B| \leqq \sum_{B \in F} \psi\left[\mathfrak{M}_{B} f\right]|B| \\
& \leqq \sum_{B \in F}|B| \mathfrak{M}_{B} \psi: f=\int_{E} \psi: f<\infty,
\end{aligned}
$$

from which follows $-\infty<S_{*}(f, \phi, E) \leqq S^{*}(f, \phi, E)<\infty$, the condition hypothesized in Theorem (5.12).

It will be shown in the next three sections that the conditions specified in this theorem are also necessary for (a), but are not necessary for (1.9).

Corollary. If $f \in L^{*}(E), \phi \in C(\Re(f))$ and $0 \leqq \phi(y)$ for $y \in \Re(f)$, the condition $\phi$ convex on $\Re(f)$ implies

$$
\int_{E} \phi: f=S(f, \phi, E, k) \leqq \infty .
$$

Proof. The function $\phi: f$ is measurable and non-negative on $E$. In case $\int_{E} \phi: f=\infty$, Theorem (5.7) implies $S(f, \phi, E, k)=S(f, \phi, E)=\infty$. In case $\int_{E} \phi: f<\infty$, the equality follows from Theorem (5.13).

6. Some lemmas. Throughout this section we assume that $E$ is a measurable linear set with

$$
|E|=\mu, \quad 0<\mu<\infty ;
$$

$f \in L^{*}(E) ; \phi$ is a non-negative function whose domain includes $\Re(f)$; and

$$
\sup _{r \in \Gamma_{\infty}(E)} \sum_{B \in F} \phi\left[\mathfrak{M}_{B} f\right]|B|=M<\infty .
$$

We begin by developing a few properties of equimeasurable functions which are needed for our special purposes and some of which may not be immediately accessible in the literature.

(6.1) Definition. Of two functions $u$ and $v$ we say that $u$ on $A$ is equimeasurable with $v$ on $B$ if and only if $A$ and $B$ are sets included, respectively, in the domains of $u$ and $v$ and the sets

$$
A \underset{t}{E}[u(t) \leqq y], \quad B \underset{E}{E}[v(s) \leqq y]
$$

are measurable and of equal measure for $-\infty<y<\infty$.

We now set

(6) See, for example, Hardy, Littlewood, and Polya, Inequalities, Cambridge, 1934, pp. 150-152. The reader should bear in mind, here and later on, that the condition $\psi$ convex on an interval implies $\psi$ continuous on the interior of that interval. 


$$
\alpha(y)=|\underset{t}{E}[f(t) \leq y]| \quad \text { for }-\infty<y<\infty,
$$

and on the open interval $(0, \mu)$ define the function $g$ by the relation

$$
g(s)=\inf \underset{y}{E}[s \leqq \alpha(y)], \quad 0<s<\mu .
$$

(6.2) LeMma. On $(0, \mu)$ the function $g$ is non-decreasing and equimeasurable with $f$ on $E$.

Proof. Obviously $g$ is non-decreasing. Let $-\infty<y_{0}<\infty$, let $a=\alpha\left(y_{0}\right)$, and set

$$
b=\left|E\left[g(s) \leqq y_{0}\right]\right|
$$

The following properties may then be readily deduced seriatim:

$$
s \leqq \alpha(g(s))
$$
for $0<s<\mu$;

$$
\begin{gathered}
g(s) \leqq y_{0} \text { implies } s \leqq \alpha(g(s)) \leqq \alpha\left(y_{0}\right)=a ; \\
g(s)>y_{0} \text { implies } s>a .
\end{gathered}
$$

Since $g$ is non-decreasing on $(0, \mu)$ we have

$$
\sup \left(\{0\}+\underset{g}{E}\left[g(s) \leqq y_{0}\right]\right)=b=\inf \left(\{\mu\}+E\left[g(s)>y_{0}\right]\right) ;
$$

this combines with (b) to yield $b \leqq a$ and with (c) to provide $b \geqq a$, whence $a=b$ and the lemma is proved.

(6.3) LEMMA. If $D$ is an open interval and $D^{\prime}$ a measurable set with $D \subset D^{\prime} \subset(0, \mu)$, and if $B^{\prime}$ is a subset of $E$ such that $f$ on $B^{\prime}$ is equimeasurable with $g$ on $D^{\prime}$, then there exists a set $B \subset B^{\prime}$ such that $f$ on $B$ is equimeasurable with $g$ on $D$ and $f$ on $B^{\prime}-B$ is equimeasurable with $g$ on $D^{\prime}-D$.

Proof. Let $(a, b)=D$, and let

$$
\begin{aligned}
& a^{\prime}=\inf _{a<t<b} g(t), \quad b^{\prime}=\sup _{a<t<b} g(t), \\
& B_{1}=B^{\prime} \underset{t}{E}\left[f(t)=a^{\prime}\right], \quad D_{1}=D \underset{g}{E}\left[g(s)=a^{\prime}\right], \\
& B_{2}=B^{\prime} \underset{t}{E}\left[a^{\prime}<f(t)<b^{\prime}\right], \quad D_{2}=D E\left[a^{\prime}<g(s)<b^{\prime}\right], \\
& B_{3}=B^{\prime} \underset{t}{E}\left[a^{\prime}<f(t)=b^{\prime}\right], \quad D_{3}=D \underset{s}{E}\left[a^{\prime}<g(s)=b^{\prime}\right] \text {. }
\end{aligned}
$$

A simple check shows that

$$
D_{2}=D^{\prime} \underset{g}{E}\left[a^{\prime}<g(s)<b^{\prime}\right]
$$


and that $f$ on $B_{2}$ is equimeasurable with $g$ on $D_{2}$. Clearly we have $\left|D_{1}\right| \leqq\left|B_{1}\right|$ and $\left|D_{3}\right| \leqq\left|B_{3}\right|$. By considering the sets

$$
(-x, x) B_{1} \text { and }(-x, x) B_{2} \quad \text { for } 0<x,
$$

we find measurable sets $\beta_{1}$ and $\beta_{3}$ with

$$
\beta_{1} \subset B_{1}, \quad \beta_{3} \subset B_{3}, \quad\left|\beta_{1}\right|=\left|D_{1}\right|, \quad\left|\beta_{3}\right|=\left|D_{3}\right| .
$$

Thus $f$ on $\beta_{1}$ is equimeasurable with $g$ on $D_{1}$ and $f$ on $\beta_{3}$ is equimeasurable with $g$ on $D_{3}$. Accordingly, on account of the disjointness of the sets involved, we may take $B=\beta_{1}+B_{2}+\beta_{3}$, note that $D=D_{1}+D_{2}+D_{3}$, and conclude that $f$ on $B$ is equimeasurable with $g$ on $D$. It then follows at once that $f$ on $B^{\prime}-B$ is equimeasurable with $g$ on $D^{\prime}-D$.

(6.4) Leмma. If $u$ on $A$ is equimeasurable with $v$ on $B$, then

$$
\int_{A} u=\int_{B} v \text { and }|A|=|B| \text {. }
$$

This well known result is easily seen.

(6.5) LemMA. If $B$ is a finite disjointed family of open subintervals of $(0, \mu)$, we have

$$
\sum_{D \in \mathfrak{S}} \phi\left[\mathfrak{M}_{D} g\right]|D| \leqq M .
$$

Proof. Let the number of intervals in $\$$ be $n$ and let

$$
B=\left\{D_{1}\right\}+\left\{D_{2}\right\}+\cdots+\left\{D_{n}\right\} .
$$

By repeated use of Lemma (6.3) we infer the existence of a disjointed family $F$ of $n$ subsets of $E$ such that

$$
F=\left\{B_{1}\right\}+\left\{B_{2}\right\}+\cdots+\left\{B_{n}\right\},
$$

with $f$ on $B_{i}$ equimeasurable with $g$ on $D_{i}(i=1,2, \cdots, n)$. From Lemma (6.4) we have

$$
\mathfrak{M}_{B_{i}} f=\mathfrak{M}_{i D_{i}} g, \quad\left|B_{i}\right|=\left|D_{i}\right| \quad(i=1,2, \cdots, n),
$$

whence

$$
\begin{aligned}
\sum_{D \in \Theta} \phi\left[\mathfrak{M}_{D} g\right]|D| & =\sum_{i=1}^{n} \phi\left[\mathfrak{M}_{D_{i}} g\right]\left|D_{i}\right|=\sum_{i=1}^{n} \phi\left[\mathfrak{M}_{B_{i}} f\right]\left|B_{i}\right| \\
& =\sum_{B \in F} \phi\left[\mathfrak{M}_{B} f\right]|B| \leqq M
\end{aligned}
$$

(6.6) LeMMA. We have $\Re(f)=\Re(g)$.

This result is almost immediate. 
(6.7) LEMMA. If $(a, b)$ is an open subinterval of $(0, \mu)$ and if $\psi$ is a function linear on an interval containing the image of $(a, b)$ under $g$ with

then we have

$$
\psi\left[\mathfrak{M}_{(a, b)} g\right]=\phi\left[\mathfrak{M}_{(a, b)} g\right]
$$

$$
\int_{a}^{b} \psi: g=\phi\left[\mathfrak{M}_{(a, b)} g\right](b-a) .
$$

Proof. Let $J$ be an interval which contains the image of $(a, b)$ under $g$ and on which $\psi$ is linear. We have

$$
\psi(y)=\omega y-\omega \gamma+\phi(\gamma) \quad \text { for } y \in J,
$$

where $\omega$ is a suitable number and $\gamma=\mathfrak{M}_{(a, b)} g$, whence

$$
\begin{aligned}
\int_{a}^{b} \psi: g=\int_{a}^{b}(\omega g(s)-\omega \gamma+\phi(\gamma)) d s & =\omega \int_{a}^{b} g-\omega \gamma(b-a)+\phi(\gamma)(b-a) \\
& =\phi(\gamma)(b-a) .
\end{aligned}
$$

(6.8) Definition. A family $K$ is irreducible if and only if

$$
\sigma(K-\{C\}) \neq \sigma(K) \quad \text { for } C \in K .
$$

(6.9) Definition. A finite irreducible family of open intervals is called a chain if and only if $\sigma(K)$ is an open interval.

Concerning chains the following remarks may be in order. No interval in a chain is the vacuous set, but the vacuous set itself constitutes a chain. The intervals in a chain may be ordered according to non-decreasing left-hand [right-hand] endpoints; these two orders are the same. Taking alternate intervals in this ordering one readily sees that a chain is expressible as the sum of two disjoint families each of which is itself disjointed. If $K$ is a chain, $K_{1} \subset K$, and $\sigma\left(K_{1}\right)$ is an interval, then $K_{1}$ is a chain. If the interval farthest to the left [right] in a chain is deleted, the remaining family is a chain. If $K$ is a chain and $C$ is an open interval which overlaps $\sigma(K)$ but contains no element of $K, K+\{C\}$ is a chain.

(6.10) Abbreviations. Let $\eta$ be a positive number fixed throughout the rest of this section. We set

$$
\begin{aligned}
W(D) & =(\eta / 2 \mu)|D|+\phi\left[\mathfrak{M}_{D} g\right]|D|, \\
\Re^{*} & =\left\{\mathfrak{M}_{(0, \mu)} g\right\}+\text { the interior of } \Re(g) .
\end{aligned}
$$

For $n$ a positive integer we employ $\langle n\rangle$ as an abbreviation for the following statement:

If $H$ is a set with $n$ elements and if

$$
\mathfrak{M}_{(0, \mu)} g \in H \subset \mathfrak{R}^{*},
$$


then there exists a non-negative convex function $\psi$ on $-\infty<y<\infty$ which dominates $\phi$ on $H$, a chain $K$ with $\sigma(K)=(0, \mu)$, and for each $D \in K$ a nonnegative function $F_{D}$ on $(0, \mu)$, all of which are so related that we have

$$
\int_{0}^{\mu} F_{D}<W(D) \quad \text { for } D \in K,
$$

and $0<s<\mu$ implies the existence of a $D \in K$ for which these conditions are satisfied:

$$
s \in D, \quad \psi[g(s)]=F_{D}(s) .
$$

(6.11) Lemma. $\langle 1\rangle$ is valid.

Proof. Let $C=(0, \mu), H=\left\{\mathfrak{M}_{C} g\right\}$, and $\psi$ be the non-negative convex function on $(-\infty, \infty)$ defined by

$$
\psi(y)=\phi\left[\mathfrak{M}_{C} g\right] \quad \text { for }-\infty<y<\infty .
$$

Let $K=\{C\}$ and define $F_{C}$ on $(0, \mu)$ by

$$
F_{C}(s)=\phi\left[\mathfrak{M}_{C} g\right] \quad \text { for } 0<s<\mu .
$$

We then have

$$
\int_{0}^{\mu} F_{C}=\mu \phi\left[\mathfrak{M}_{C} g\right]=\phi\left[\mathfrak{M}_{C} g\right]|C|<W(C),
$$

and $0<s<\mu$ implies

$$
F_{C}(s)=\phi\left[\mathfrak{M}_{C} g\right]=\psi[g(s)] .
$$

(6.12) LEMMA. If $\phi$ is continuous on $\Re(f)$ and $n$ is a positive integer, the validity of $\langle n\rangle$ implies that of $\langle n+1\rangle$.

Proof. Let $H$ be a set with $n+1$ elements and assume

$$
\mathfrak{M}_{(0, \mu)} g \in H \subset \mathfrak{R}^{*} \text {. }
$$

Either there is a number in $H$ which is $>\mathfrak{M}_{(0, \mu)} g$ or there is a number in $H$ which is $\left\langle\mathfrak{M}_{(0, \mu)}\right.$ g. For definiteness we assume henceforth that the former is the case and we designate by $\nu$ the largest such number. It should be clear from the nature of the ensuing argument that analogous reasoning can be brought to bear on the alternative case.

Let

$$
\bar{H}=H-\{\nu\},
$$

so that $\bar{H}$ is a set of $n$ elements and

$$
\mathfrak{M}_{(0, \mu)} g \in \bar{H} \subset \Re^{*} \text {. }
$$

In view of the validity of $\langle n\rangle$, let $\bar{\psi}$ be a non-negative convex function on $(-\infty, \infty)$ which dominates $\phi$ on $\bar{H}$; let $\bar{K}$ be a chain with $\sigma(\bar{K})=(0, \mu)$; 
and for each $D \in \bar{K}$ let $\bar{F}_{D}$ be a non-negative function on $(0, \mu)$, all of which are so related that we have

$$
\int_{0}^{\mu} F_{D}<W(D) \quad \text { for } D \in \bar{K}
$$

and

(b)

$$
0<s<\mu \text { implies the existence of a } D \in \bar{K} \text { with }
$$

$$
s \in D, \quad \bar{\psi}[g(s)]=\bar{F}_{D}(s) .
$$

In the case $\phi(\nu) \leqq \bar{\psi}(\nu)$ it is apparent that $\langle n+1\rangle$ holds. From now on we assume

$$
\bar{\psi}(\nu)<\phi(\nu) .
$$

Recalling that $\mathfrak{M}_{(0, \mu)} g$ is $<\nu$ we let $\xi$ satisfy the conditions

$$
0<\xi<\mu, \quad \mathfrak{M}_{(\xi, \mu)} g=\nu .
$$

Let $C_{1}$ be the interval farthest to the right in $\bar{K}$. By moving only the right endpoint of $C_{1}$ a suitably small amount to the left we obtain an interval $C_{2}$ which not only enjoys the property that its right endpoint is interior to the interval

$$
(\xi, \mu)-\sigma\left(\bar{K}-\left\{C_{1}\right\}\right)
$$

but also, in view of the continuity of $\phi$, the property

$$
\int_{0}^{\mu} \bar{F}_{C_{1}}<W\left(C_{2}\right)
$$

Recalling (a) and defining

$$
\bar{F}_{C_{2}}=\bar{F}_{C_{1}}, \quad \bar{K}_{1}=\bar{K}-\left\{C_{1}\right\}+\left\{C_{2}\right\},
$$

we see at once

$$
\int_{0}^{\mu} \bar{F}_{D}<W(D) \quad \text { for } D \in \bar{K}_{1} \text {. }
$$

Our determination of $C_{2}$ insures that $\bar{K}_{1}$ is a chain in which the interval farthest to the right is $C_{2}$, whence

(g)

$$
s \in \sigma\left(\bar{K}_{1}\right) C_{1} \text { implies } s \in C_{2} \text { 。 }
$$

Making use of (b), the first relation in (e), and (g) we infer

$$
s \in \sigma\left(\bar{K}_{1}\right) \text { implies the existence of a } D \in \bar{K}_{1} \text { with }
$$

$$
s \in D, \quad \bar{\psi}[g(s)]=\bar{F}_{D}(s) .
$$


Setting

$$
\begin{aligned}
\bar{K}_{2} & =\bar{K}_{1}-\bar{K}_{1} \underset{D}{E}[D \subset(\xi, \mu)], \\
K & =\bar{K}_{2}+\{(\xi, \mu)\},
\end{aligned}
$$

we note that $\bar{K}_{2}$ and $K$ are chains and $\sigma(K)=(0, \mu)$. Since $\nu$ is interior to $\Re(g)$ we obtain from (d) the inequality $g(\xi)<\nu$. Clearly there exists a function $\psi$ on $(-\infty, \infty)$ with

$$
\begin{array}{rlr}
\psi(y)=\bar{\psi}(y) & \text { for } y \leqq g(\xi), \\
\psi \text { linear on } \underset{y}{E}[g(\xi) \leqq y], \quad \psi(\nu)=\phi(\nu) . &
\end{array}
$$

Let

$$
F_{D}=\bar{F}_{D}
$$

for $D \in \bar{K}_{2}$;

for $D=(\xi, \mu)$.let $F_{D}$ be defined on $(0, \mu)$ by the relations

$$
\begin{array}{ll}
F_{D}(s)=\psi[g(s)] & \text { for } \xi<s<\mu, \\
F_{D}(s)=0 & \text { for } 0<s \leqq \xi .
\end{array}
$$

By aid of (c) we find

$$
\bar{\psi}(\nu)<\phi(\nu)=\psi(\nu) ;
$$

from this relation and (i) it follows that $\psi$ is non-negative and convex on $(-\infty, \infty)$ with

$$
\bar{\psi}(y) \leqq \psi(y) \quad \text { for } y \leqq \nu .
$$

Thus $\psi$ dominates $\phi$ not only on $\bar{H}$, since each number in $\bar{H}$ is $\langle\nu$, but on $H$ as well.

From (k), (d), (i), Lemma (6.7), and Abbreviations (6.10) we see that for $D=(\xi, \mu)$ we have

$$
\int_{0}^{\mu} F_{D}=\int_{\xi}^{\mu} F_{D}=\int_{\xi}^{\mu} \psi: g=\phi\left[\mathfrak{M}_{(\xi, \mu)} g\right](\mu-\xi)=\phi\left[\mathfrak{M}_{D} g\right]|D|<W(D) .
$$

For $D=(\xi, \mu)$ the validity of the relation

$$
\int_{0}^{\mu} F_{D}<W(D)
$$

is thus evident; that it is valid for $D \in \bar{K}_{2}$ is clear from (f) and the relation $\bar{K}_{2} \subset \bar{K}_{1}$. From the definition of $K$ we then have relation (l) for $D \in K$.

For $0<s \leqq \xi$ we have $s \in \sigma\left(\bar{K}_{2}\right) \subset \sigma\left(\bar{K}_{1}\right)$, and by (h) there exists $D \in \bar{K}_{1}$ with

$$
s \in D, \quad \bar{\psi}[g(s)]=\bar{F}_{D}(s) ;
$$

for this particular $D$ it is clear that we have

$$
D \in \bar{K}_{2} \subset K, \quad g(s) \leqq g(\xi),
$$


and hence, by aid of (i) and (j),

$$
s \in D, \quad \psi[g(s)]=F_{D}(s) .
$$

The proof that $0<s<\mu$ implies the existence of a $D \in K$ with property (m) is now readily completed by reference to $(\mathrm{k})$.

The proof of the lemma is now complete.

(6.13) Lemma. If $\phi$ is continuous on $\Re(f), H$ is a finite subset of the interior of $\Re(f)$, and $\eta$ is an arbitrary positive number, there exists a non-negative convex function $\psi$ on $(-\infty, \infty)$ which dominates $\psi$ on $H$ and satisfies the condition

$$
\int_{E} \psi: f<\eta+2 M
$$

Proof. Let $H^{*}=H+\left\{\mathfrak{M}_{E} f\right\}$. From Lemmas (6.4) and (6.6) we have

$$
\mathfrak{M}_{(0, \mu)} g=\mathfrak{M}_{E} f, \quad \mathfrak{R}(g)=\mathfrak{R}(f) ;
$$

hence, from the definition of $\Re^{*}$ in (6.10), we see that $H^{*}$ is a finite set with

$$
\mathfrak{M}_{(0, \mu)} g \in H^{*} \subset \mathfrak{R}^{*} \text {. }
$$

Lemmas (6.11) and (6.12) insure the existence of a non-negative convex function $\psi$ which dominates $\phi$ on $H^{*}$, of a chain $K$ with $\sigma(K)=(0, \mu)$, and for each $D \in K$ a non-negative function $F_{D}$ on $(0, \mu)$, all so related as to have the properties specified in $\langle n\rangle$ under (6.10):

$$
\int_{0}^{\mu} F_{D}<W(D) \quad \text { for } D \in K,
$$

(b) $\quad 0<s<\mu$ implies the existence of a $D \in K$ with

$$
s \in D, \quad \psi[g(s)]=F_{D}(s) .
$$

It was pointed out in the remarks following Definition (6.9) that we may write

$$
K=\mathfrak{B}_{1}+\mathbb{S}_{2},
$$

where $\mathscr{B}_{1}$ is a disjointed family of intervals in $K, \mathfrak{B}_{2}$ is likewise, and $\mathscr{G}_{1} \mathscr{G}_{2}$ is vacuous. Thus, using (6.10) and Lemma (6.5), we have

$$
\begin{aligned}
\sum_{D \in K} W(D)= & \sum_{D \in \mathbb{S}_{1}} W(D)+\sum_{D \in \mathbb{G}_{2}} W(D) \\
\leqq & (\eta / 2 \mu) \sum_{D \in \mathbb{S}_{1}}|D|+(\eta / 2 \mu) \sum_{D \in \mathbb{G}_{2}}|D|+\sum_{D \in \mathbb{G}_{1}} \phi\left[\mathfrak{M}_{D} g\right]|D| \\
& +\sum_{D \in \mathbb{S}_{2}} \phi\left[\mathfrak{M}_{D} g\right]|D| \\
\leqq & (\eta / 2 \mu) \mu+(\eta / 2 \mu) \mu+M+M=\eta+2 M .
\end{aligned}
$$


The relation

$$
\psi[g(s)] \leqq \sum_{D \in K} F_{D}(s) \quad \text { for } 0<s<\mu
$$

is an immediate consequence of (b) and the non-negativity of $F_{D}$. These last two inequalities now combine with (a) to yield

(c) $\quad \int_{0}^{\mu} \psi: g \leqq \int_{0}^{\mu}\left(\sum_{D \in K} F_{D}\right)=\sum_{D \in K} \int_{0}^{\mu} F_{D}<\sum_{D \in K} W(D) \leqq \eta+2 M$.

Since $g$ on $(0, \mu)$ is equimeasurable with $f$ on $E$ and $E_{y}[\psi(y) \leqq a]$ is an interval for $-\infty<a<\infty$, it is readily shown that $\psi: g$ on $(0, \mu)$ is equimeasurable with $\psi: f$ on $E$. Accordingly, from Lemma (6.3) and (c) we have

$$
\int_{E} \psi: f=\int_{0}^{\mu} \psi: g<\eta+2 M
$$

Since $\psi$ dominates $\phi$ on $H^{*}$, it dominates $\phi$ on $H$. The proof is complete.

(6.14) LEMMA. If $J$ is an interval and for each positive integer $n$ the function $\psi_{n}$ is non-negative and convex on $J$ with

$$
\limsup _{n \rightarrow \infty} \psi_{n}(t)<\infty \quad \text { for } t \in J,
$$

then there exists a function $\psi_{0}$ non-negative and convex on $J$ and integers $N_{i}$ $(i=1,2,3, \cdots)$ with the properties

$$
\dot{1} \leqq N_{1}<N_{2}<N_{3}<\cdots, \lim _{i \rightarrow \infty} \psi_{N_{i}}(t)=\psi_{0}(t) \quad \text { for } t \in J .
$$

Proof. Let $J_{m}=\left[a_{m}, b_{m}\right](m=1,2,3, \cdots)$ be a sequence of closed intervals with

$$
J_{m} \subset J_{m+1} \quad(m=1,2,3, \cdots), \quad \sum_{m=1}^{\infty} J_{m}=J
$$

and let

$$
C=\sum_{n=1}^{\infty}\left\{\psi_{n}\right\}
$$

For each pair of positive integers $m$ and $n$ the total variation of $\psi_{n}$ on $J_{m}$ is less than or equal to $\psi_{n}\left(a_{m}\right)+\psi_{n}\left(b_{m}\right)$, a number which is also a bound for $\psi_{n}$ on $J_{m}$. Thus there exists a sequence of non-negative numbers $M_{m}$ $(m=1,2,3, \cdots)$ such that for each positive integer $m$ and each $\psi \in C$ the total variation of $\psi$ on $J_{m}$, and the values of $\psi$ on $J_{m}$, do not exceed $M_{m}$. From a theorem of Helly $\left({ }^{7}\right)$ we conclude that for each positive integer $m$ the

(7) Helly, Über lineare Funktionaloperationen, Sitzungsberichte der Akademie der Wissenschaften in Wien vol. 121, IIa (1912) p. 283. 
family $C$ is conditionally compact in the topology of pointwise convergence on $J_{m}$; that is, each sequence of elements of $C$ contains a subsequence which is pointwise convergent on $J_{m}$.

Using a familiar diagonal process of selection and the properties (a) of the intervals $J_{m}$, we see that in the topology of pointwise convergence on $J$ the family $C$ is also conditionally compact. Hence there exist integers $N_{i}$ $(i=1,2,3, \cdots)$ with $1 \leqq N_{1}<N_{2}<N_{3}<\cdots$ such that

$$
\lim _{t \rightarrow \infty} \psi_{N_{i}}(t) \text { exists (finite) } \quad \text { for } t \in J \text {. }
$$

Defining $\psi_{0}$ on $J$ as this limit, it follows at once that $\psi_{0}$ is non-negative and convex on $J$.

7. Concerning convex dominants for the function $\phi$. In this section $E$ is to be understood as a measurable linear set with

$$
|E|=\mu, \quad 0<\mu \leqq \infty .
$$

(7.1) ThEOREM. If $f \in L^{*}(E), \phi$ is a non-negative continuous function on $\Re(f)$, and

$$
\sup _{F \in \Gamma_{\infty}(E)} \sum_{B \in F} \phi\left[\mathfrak{M}_{B} f\right]|B|=M<\infty,
$$

then there exists a non-negative convex function $\psi$ which dominates $\phi$ on $\Re(f)$, with

$$
\int_{E} \psi: f \leqq 2 M
$$

Proof. If the interior of $\Re(f)$ is vacuous, $\Re(f)$ consists of but a single point; in this case $\psi$ may be taken as the non-negative convex function $\psi(y)=\phi(y)$ for $y \in \Re(f)$ and the conclusion is apparent. Accordingly we assume from now on that the interior of $\Re(f)$ is not vacuous.

Let $m>0$ be such that the condition

$$
|[-m, m] E|>0
$$

is satisfied. For each positive integer $n$ let

let

$$
E_{n}=[-m n, m n] E ;
$$

$$
\left\{r_{1}\right\}+\left\{r_{2}\right\}+\left\{r_{3}\right\}+\cdots
$$

be the set of all rational numbers; let $f_{n}$ be the function defined on $E_{n}$ by

$$
f_{n}(t)=f(t) \quad \text { for } t \in E_{n} ;
$$

let

$$
\left.H_{n}=\text { [interior of } \Re\left(f_{n}\right)\right]\left[\left\{r_{1}\right\}+\left\{r_{2}\right\}+\cdots+\left\{r_{n}\right\}\right] \text {; }
$$

and let $h_{n}$ be the characteristic function of $E_{n}$. Finally, let 


$$
H=\sum_{n=1}^{\infty} H_{n}
$$

We see at once the relations

$$
\begin{aligned}
& \lim _{n \rightarrow \infty} h_{n}(t)=1 \\
& \text { for } t \in E \text {, } \\
& \text { (c) } \quad \Re\left(f_{n}\right) \subset \Re\left(f_{n+1}\right), \quad H_{n} \subset H_{n+1} \quad(n=1,2,3, \cdots) \text {. }
\end{aligned}
$$

A more careful check shows

(e)

$$
\begin{gathered}
\Re(f)=\sum_{n=1}^{\infty} \Re\left(f_{n}\right), \\
\bar{H} \supset \Re(f),
\end{gathered}
$$

where $\bar{H}$ represents the closure of $H$.

For each positive integer $n$ we have

$$
\sup _{F \in \Gamma_{\infty}\left(E_{n}\right)} \sum_{B \in F} \phi\left[\mathfrak{M}_{B} f_{n}\right]|B| \leqq \sup _{F \in \Gamma_{\infty}(E)} \sum_{B \in F} \phi\left[\mathfrak{M}_{B} f\right]|B|=M,
$$

and $H_{n}$ is a finite subset of $\Re\left(f_{n}\right)$; by Lemma (6.13) there exists a non-negative convex function $\psi_{n}$ on $(-\infty, \infty)$ which dominates $\phi$ on $H_{n}$ and satisfies the inequality

$$
\int_{E_{n}} \psi_{n}: f_{n} \leqq 2 M+1 / n
$$

Let $y_{0} \in \Re(f)$. Recalling (c) and (d) we let $n_{0}$ be such that $y_{0} \in \Re\left(f_{n}\right)$ for $n \geqq n_{0}$. Let $B_{0}$ be a subset of $E_{n_{0}}$ with $\mathfrak{M}_{B_{0}} f_{n_{0}}=y_{0}$. From the definition of $f_{n}$ and the relations

$$
E_{n} \subset E_{n+1} \quad(n=1,2,3, \cdots)
$$

follows

$$
y_{0}=\mathfrak{M}_{B_{0}} f=\mathfrak{M}_{B_{0}} f_{n} \quad \text { for } n \geqq n_{0},
$$

and, by aid of Jensen's inequality,

whence

$$
\psi_{n}\left(y_{0}\right)=\psi_{n}\left[\mathfrak{M}_{B_{0}} f_{n}\right] \leqq \mathfrak{M}_{B_{0}} \psi_{n}: f_{n} \leqq \frac{2 M+1 / n}{\left|B_{0}\right|} \quad \text { for } n \geqq n_{0},
$$

$$
\limsup _{n \rightarrow \infty} \psi_{n}\left(y_{0}\right) \leqq 2 M /\left|B_{0}\right|<\infty .
$$

By Lemma (6.14) we now infer the existence of a non-negative convex function $\psi$ on $\Re(f)$ and a sequence of integers $N_{i}(i=1,2,3, \cdots)$ with the properties

$$
1 \leqq N_{1}<N_{2}<N_{3}<\cdots, \lim _{i \rightarrow \infty} \psi_{N_{i}}(y)=\psi(y) \quad \text { for } y \in \Re(f) .
$$


Since $f(x) \in \Re(f)$ for almost all $x \in E$, it may be inferred by aid of (b) that

$$
\lim _{i \rightarrow \infty} h_{N_{i}}(x) \psi_{N_{i}}[f(x)]=\lim _{i \rightarrow \infty} \psi_{N_{i}}[f(x)]=\psi[f(x)]
$$

for almost all $x \in E$.' Using a well known theorem of Fatou we then obtain

$$
\begin{aligned}
\int_{E} \psi: f & \leqq \liminf _{i \rightarrow \infty} \int_{E} h_{i}(x) \psi_{N_{i}}[f(x)] d x=\liminf _{i \rightarrow \infty} \int_{E_{N_{i}}} \psi_{N_{i}}: f \\
& =\liminf _{i \rightarrow \infty} \int_{E_{N_{i}}} \psi_{N_{i}}: f_{N_{i}}=\lim _{i \rightarrow \infty} \int_{E_{N_{i}}} \psi_{N_{i}}: f_{N_{i}} \\
& \leqq \lim _{i \rightarrow \infty}\left(2 M+1 / N_{i}\right)=2 M .
\end{aligned}
$$

From the second part of (c) and (a) it follows that $\psi$ dominates $\phi$ on $H$. From (e), the fact that the convex function $\psi$ is upper semi-continuous on $\Re(f)$, and the continuity of $\phi$, we now conclude that $\psi$ dominates $\phi$ on $\Re(f)$.

REMARK. In Theorem (7.1) the hypothesis that $\phi$ is continuous can be dispensed with. To show this in detail appears to be a tedious process, but it may be indicated roughly as follows. Employing properties of equimeasurable functions as given in $\S 6$, Theorem (7.1) may be used to establish this result:

If $f \in L^{*}(E), \phi$ is a non-negative function on $\Re(f)$,

$$
\sup _{F \in \Gamma_{\infty}(E)} \sum_{B \in F} \phi\left[\mathfrak{M}_{B} f\right]|B|=M .<\infty,
$$

$H$ is a finite subset of $\Re(f)$, and $\eta$ is an arbitrary positive number, there exists a non-negative convex function $\psi$ on $(-\infty, \infty)$ which dominates $\phi$ on $H$ and satisfies the condition

$$
\int_{E} \psi: f \leqq \eta+2 M
$$

Using this fact and suitably redefining $H_{n}$, as it was introduced in the proof of Theorem (7.1), we may reach the desired conclusion. It is then easy to remove from Theorem (7.3) below the hypothesis that $\phi$ is continuous.

(7.2) THEOREM. If $f \in L^{*}(E), \phi$ is a non-negative continuous function on $\Re(f)$, and

$$
\sup _{P \in \Gamma_{\infty}(E)} \sum_{B \in F} \phi\left[\mathfrak{M}_{B} f\right]|B|=M<\infty,
$$

there exists a non-negative continuous convex function $\psi$ which dominates $\phi$ on $\Re(f)$, with

$$
\int_{E} \psi: f \leqq 2 M
$$

Proof. By Theorem (7.1) there exists a non-negative convex function $\psi_{0}$ 
which dominates $\phi$ on $\Re(f)$, with $\int_{E} \psi_{0}: f \leqq 2 M$. If the interior of $\Re(f)$ is vacuous, $\psi_{0}$ may be taken as $\psi$. Otherwise let $\psi$ be the continuous function on $\Re(f)$ which is identical with $\psi_{0}$ on the interior of $\Re(f)$. Since $\phi$ is continuous and $\psi_{0}$ is convex, we have

$$
\phi(y) \leqq \psi(y) \leqq \psi_{0}(y)
$$

for $y \in \Re(f)$

and the conclusion is immediate.

(7.3) THEOREM. If $f \in L^{*}(E), \phi \in C(\Re(f))$, and

$$
\sup _{r \in \Gamma_{\infty}(E)}\left|\sum_{B \in F} \phi\left[\mathfrak{M}_{B} f\right]\right| B||<\infty,
$$

there exists a non-negative continuous convex function $\psi$ which dominates $|\phi|$ on $\Re(f)$, with

$$
\int_{E} \psi: f<\infty
$$

Proof. Defining the function $\Phi$ on $\Re(f)$ by

$$
\Phi(y)=|\phi(y)|
$$

we infer from Theorem (3.12)

$$
\sup _{r \in \Gamma_{\infty}(E)} \sum_{B \in F} \Phi\left[\mathfrak{M}_{B} f\right]|B|<\infty .
$$

The conclusion then follows from Theorem (7.2).

It is almost superfluous to remark that in Theorems (7.1)-(7.3), if the set $E$ is essentially bounded, we have $L^{*}(E)=L(E)$ and $\Gamma_{\infty}(E)$ can be replaced by $\Gamma_{\eta}(E)$ where $\eta$ represents the essential diameter of $E$.

8. Certain counterexamples. In order that the question at issue should be completely covered, the theorems of $\$ 7$ above must be supplemented by an example to show that for $f \in L^{*}(E)$ and $\phi \in C(\Re(f))$ the condition that $|\phi|$ be dominated by a convex function as specified in Theorem (5.13) is not necessary for (1.9). In this section we shall give two examples and conclude with a few supplementary remarks.

ExAMPLE A. The following example exhibits a function $f \in L(I)$ and a non-negative function $\phi \in C(\Re(f))$ for which relation (1.9) holds but $\phi$ is dominated on $\Re(f)$ by no convex function $\psi$ with $\int_{r} \psi: f<\infty$.

Let $\phi_{1}$ be defined on $(-\infty, \infty)$ thus : for $-\infty<y \leqq 0, \phi_{1}(y)=0$; for $n \geqq 1$ and odd, $\phi_{1}(2 n)=2^{(2 n)^{2}}, \phi_{1}(2 n+2)=2^{(2 n+3)^{2}} ;$ for $n \geqq 2$ and even, $\phi_{1}(2 n)=2^{(2 n+1)^{2}}$, $\phi_{1}(2 n+2)=2^{(2 n+2)^{2}}$; on each closed interval $[2 n, 2 n+2](n=0,1,2, \cdots)$, $\phi_{1}$ is linear. Let $\phi_{2}$ be defined in the same manner except for interchange of the specifications when $n$ is odd and when $n$ is even..It may be verified at once that the slope of $\phi_{i}(i=1,2)$ on the interval $[2 n, 2 n+2]$ is an increasing 
function of $n$; thus $\phi_{i}$ is convex on $(-\infty, \infty)$. For each $y \in(-\infty, \infty)$ let $\phi(y)=\min \left[\phi_{1}(y), \phi_{2}(y)\right]$, so that $\phi$ is continuous on $(-\infty, \infty)$.

Let $I_{n}(n=1,2,3, \cdots)$ be a sequence of disjoint intervals with $\left|I_{n}\right|=1 / 2^{(2 n)^{2}+n}, I_{n} \subset[2 / 3,1]$ for $n$ odd, and $I_{n} \subset[0,1 / 3]$ for $n$ even. Let the functions $f_{1}, f_{2}$, and $f$ be defined on $I$ by

$$
\begin{aligned}
& f_{1}(x)=\left\{\begin{array}{l}
2 n \text { for } x \in I_{n} \text { and } n \text { odd, } \\
0 \text { otherwise, }
\end{array} f_{2}(x)=\left\{\begin{array}{l}
2 n \text { for } x \in I_{n} \text { and } n \text { even, } \\
0 \text { otherwise }
\end{array}\right.\right. \\
& f(x)=f_{1}(x)+f_{2}(x) \quad \text { for } x \in I .
\end{aligned}
$$

One verifies at once $f_{1} \in L(I), f_{2} \in L(I)$, and hence $f \in L(I)$.

Under the condition diam $(B)<\delta \leqq 1 / 3$, which we now assume to be in force, a set $B$ which contains a point of either interval $[0,1 / 3],[2 / 3,1]$ contains no point of the other. From the definitions of $f_{1}$ and $f_{2}$ it is therefore clear that

$$
\int_{A B} f_{1}>0 \text { implies } \int_{A B} f_{2}=0
$$

and vice versa. Thus, for $F \in \Gamma_{\delta}(I)$, we have

as well as

$$
\begin{aligned}
\sum_{B \in F} \phi\left[\mathfrak{M}_{A B} f\right]|B| & =\sum_{B \in F} \phi\left[\mathfrak{M}_{A B} f_{1}+\mathfrak{M}_{A B} f_{2}\right]|B| \\
& =\sum_{B \in F} \phi\left[\mathfrak{M}_{A B} f\right]|B|+\sum_{B \in F} \phi\left[\mathfrak{M}_{A B} f\right]|B|,
\end{aligned}
$$

$$
\int_{B} \phi: f=\int_{B} \phi:\left(f_{1}+f_{2}\right)=\int_{B} \phi: f_{1}+\int_{B} \phi: f_{2} \quad \text { for } B \in F .
$$

We may then infer from Theorem (5.13)

$$
\begin{aligned}
\lim _{\delta \rightarrow 0} \sup _{F \in \Gamma_{\delta}(I)} & \sum_{B \in F}\left|\mathfrak{M}_{B} \phi: f-\phi\left[\mathfrak{M}_{A B} f\right]\right||B| \\
= & \lim _{\delta \rightarrow 0} \sup _{r \in \Gamma_{\delta}(I)} \sum_{B \in F}\left|\mathfrak{M}_{B} \phi: f_{1}-\phi\left[\mathfrak{M}_{A B} f_{1}\right]\right||B| \\
& +\lim _{\delta \rightarrow 0} \sup _{r \in \Gamma_{\delta}(I)} \sum_{B \in F}\left|\mathfrak{M}_{B} \phi: f_{2}-\phi\left[\mathfrak{M}_{A B} f_{2}\right]\right||B|=0,
\end{aligned}
$$

since $\phi$ is dominated by the convex function $\phi_{i}$ with

$$
\int_{I} \phi_{i}: f_{i}<\sum_{n=1}^{\infty} 2^{(2 n)^{2} / 2^{(2 n)^{2}+n}}=1 \quad(i=1,2) .
$$

We shall now show that if $\psi$ is any convex function dominating $\phi$ on $(-\infty, \infty), \int_{I} \psi: f$ does not exist (finite). Let $n$ be odd and $\geqq 1$ and consider the interval $[2 n, 2 n+2]$. On this closed interval $\phi_{1}$ and $\phi_{2}$ are linear, with derivatives that we may designate, respectively, by $\mu_{1}$ and $\mu_{2}\left(\mu_{1}>\mu_{2}\right)$, and their graphs intersect at a point $(\alpha, \beta), 2 n<\alpha<2 n+2$. On the subintervals 
$[2 n, \alpha]$ and $[\alpha, 2 n+2]$ we have respectively $\phi=\phi_{1}$ and $\phi=\phi_{2}$. The function $\zeta$ defined thus on $(-\infty, \infty)$,

$$
\zeta(y)=\left\{\begin{array}{l}
\phi_{1}(y) \text { for } 2 n \leqq y \leqq \alpha, \\
\text { linear for }-\infty<y \leqq \alpha, \\
\phi_{2}(y) \text { for } \alpha \leqq y \leqq 2 n+2, \\
\text { linear for } \alpha \leqq y<\infty,
\end{array}\right.
$$

is concave on $(-\infty, \infty)$, with $\zeta(y) \leqq \psi(y)$ for $-\infty<y<\infty$. Hence there exists a function $\phi^{*}$ linear on $[2 n, 2 n+2]$, with derivative $\mu_{0}$ and with $\phi^{*}(\alpha)=\beta$, satisfying the conditions

$$
\begin{aligned}
& \mu_{1} \geqq \mu_{0} \geqq \mu_{2}, \\
& \phi^{*}(y) \leqq \psi(y) \quad \text { for } 2 n \leqq y \leqq 2 n+2 .
\end{aligned}
$$

Now a line through the point $(\alpha, \beta)$ with slope $\mu$ cuts the verticals through $(2 n, 0)$ and $(2 n+2,0)$, respectively, in points whose ordinates are

$$
h_{1}(\mu)=\beta+\mu(2 n-\alpha), \quad h_{2}(\mu)=\beta+\mu(2 n+2-\alpha) .
$$

From the condition $\psi(y) \geqq \phi(y) \geqq 0$ on $-\infty<y<\infty$, we infer

$$
\begin{aligned}
\int_{I} \psi: f & >\int_{I_{n}} \psi: f+\int_{I_{n}+1} \psi: f=\psi(2 n) / 2^{(2 n)^{2}+n}+\psi(2 n+2) / 2^{(2 n+2)^{2}+n+1} \\
& \geqq \phi^{*}(2 n) / 2^{(2 n)^{2}+n}+\phi^{*}(2 n+2) / 2^{(2 n+2)^{2}+n+1} \\
& =h_{1}\left(\mu_{0}\right) / 2^{(2 n)^{2}+n}+h_{2}\left(\mu_{0}\right) / 2^{(2 n+2)^{2}+n+1}=H\left(\mu_{0}\right)
\end{aligned}
$$

where

$$
H(\mu)=h_{1}(\mu) / 2^{(2 n)^{2}+n}+h_{2}(\mu) / 2^{(2 n+2)^{2}+n+1} .
$$

Since $H$ is a linear function of $\mu$, at least one of the inequalities

$$
H\left(\mu_{0}\right) \geqq H\left(\mu_{1}\right), \quad H\left(\mu_{0}\right) \geqq H\left(\mu_{2}\right)
$$

holds. But, since no one of the numbers $h_{i}\left(\mu_{j}\right)(i, j=1,2)$ can be negative, we have

$$
\begin{aligned}
& H\left(\mu_{1}\right) \geqq h_{2}\left(\mu_{1}\right) / 2^{(2 n+2)^{2}+n+1}=2^{(2 n+3)^{2}} / 2^{(2 n+2)^{2}+n+1}=2^{3 n+4}, \\
& H\left(\mu_{2}\right) \geqq h_{1}\left(\mu_{2}\right) / 2^{(2 n)^{2}+n}=2^{(2 n+1)^{2}} / 2^{(2 n)^{2}+n}=2^{3 n+1},
\end{aligned}
$$

whence

$$
\int_{I} \psi: f>2^{3 n+1}
$$

and the fact that $\int_{I} \psi: f$ does not exist (finite) follows from the arbitrariness of the odd integer $n$. 
Another question which it is natural to raise is answered by

Example B. The following example shows that in Theorem (5.13) the hypothesis that $|\phi|$ be dominated by a convex function $\psi$ cannot be weakened to the hypothesis that $|\phi|$ be dominated by a strictly monotone function $\psi$ even if one takes $E=I$ and insists that $f$ be continuous on $I$ save at one point, that no sampling be indulged in, and that the restriction $F \in \bar{\Gamma}_{\delta}(I)$ be imposed.

Our example is one in which $f$ is non-negative on $I$ and $\phi$ itself is positive, continuous, and strictly continuous on the interval $0 \leqq y<\infty$, with $\int_{I} \phi: f<\infty$. In order that our purposes later in $\$ 11$ may also be served we construct this example to satisfy the additional condition

$$
\lim _{y \rightarrow \infty} \phi(y) / y=\infty \text {. }
$$

To fit completely into the situation of $\$ 11$ we should extend the interval on which $\phi$ is defined to $(-\infty, \infty)$ by setting

$$
\phi(y)=16+y^{2} \quad \text { for }-\infty<y<0,
$$

for example, so that $\phi$ is continuous and positive on $(-\infty, \infty)$ with $\lim _{|y| \rightarrow \infty} \phi(y) /|y|=\infty$.

Let

$$
a_{n}=1 / 2^{22 n}, \quad m_{n}=\left(a_{n+1}\right)^{1 / 2} \quad(n=1,2,3, \cdots),
$$

so that $\lim _{n \rightarrow \infty} a_{n}=0$ and for each $n$ we have

$$
a_{n+1}=a_{n}^{4}, \quad m_{n}^{2}=a_{n+1}, \quad m_{n}=a_{n}^{2}, \quad a_{n+1}<m_{n}<a_{n} \leqq a_{1}=1 / 16 .
$$

On $I$ let $g$ be defined by the conditions

and let

$$
\begin{aligned}
& g(0)=0, \quad g(x)=1 \\
& g(x)=\left\{\begin{array}{lll}
m_{n}^{-5 / 4} & \text { for } a_{n+1}<x \leqq m_{n}, & \text { for } 1 / 16<x \leqq 1, \\
1 & \text { for } m_{n}<x \leqq a_{n},
\end{array}\right.
\end{aligned}
$$

$$
f(0)=0, \quad f(x)=\int_{x}^{1} g \quad \text { for } 0<x \leqq 1 .
$$

The function $f$ is then absolutely continuous on every interval $[\epsilon, 1], \epsilon>0$, but the relation

$$
\begin{aligned}
\int_{a_{n+1}}^{m_{n}} g & =m_{n}^{-5 / 4}\left(m_{n}-a_{n+1}\right)=m_{n}^{-5 / 4}\left(m_{n}-m_{n}^{2}\right) \\
& =m_{n}^{-1 / 4}\left(1-m_{n}\right)>(15 / 16) m_{n}^{-1 / 4} \quad(n=1,2,3, \cdots)
\end{aligned}
$$

implies $\lim _{x \rightarrow 0} f(x)=\infty$.

The inequality 


$$
\int_{a_{n+1}}^{a_{n}} f /\left(a_{n}-a_{n+1}\right)>f\left(m_{n}\right) \quad(n=1,2,3, \cdots)
$$

may be shown as follows. For arbitrary $n$ let

$$
a=a_{n+1}, \quad c=m_{n}, \quad b=a_{n} .
$$

Then we have

whence

$$
\begin{aligned}
f(x) & =f(b)+\int_{x}^{b} g=f(b)+(b-x) \quad \text { for } c \leqq x \leqq b, \\
f(c) & =f(b)+(b-c)
\end{aligned}
$$

and

$$
\int_{c}^{b} f=(b-c) f(b)+(b-c)^{2} / 2=(b-c) f(c)-(b-c)^{2} / 2 ;
$$

$$
\begin{aligned}
& f(x)=f(c)+\int_{x}^{c} g=f(c)+c^{-5 / 4}(c-x) \quad \text { for } a \leqq x \leqq c, \\
& \int_{a}^{c} f=(c-a) f(c)+c^{-5 / 4}(c-a)^{2} / 2 .
\end{aligned}
$$

Thus we obtain

$$
\int_{a}^{b} f=(b-a) f(c)+\left[c^{-5 / 4}(c-a)^{2}-(b-c)^{2}\right] / 2,
$$

where

$$
\begin{aligned}
c^{-5 / 4}(c-a)^{2}-(b-c)^{2} & =c^{-5 / 4}\left(c-c^{2}\right)^{2}-(b-c)^{2}>c^{3 / 4}(1-c)^{2}-b^{2} \\
& =c^{3 / 4}(1-c)^{2}-c>c^{3 / 4}(15 / 16)^{2}-c \\
& =c^{3 / 4}\left[(15 / 16)^{2}-c^{1 / 4}\right] \\
& >c^{3 / 4}\left[(15 / 16)^{2}-(1 / 16)^{1 / 4}\right]>0 .
\end{aligned}
$$

From the definition of $g$ we have

whence

$$
0<g(x) \leqq x^{-5 / 4} \quad \text { for } 0<x \leqq 1,
$$

$$
0 \leqq f(x)=\int_{x}^{1} g \leqq \int_{x}^{1} t^{-5 / 4} d t<4 x^{-1 / 4} \quad \text { for } 0<x \leqq 1 .
$$

Thus we infer $f \in L(I)$. Moreover, as $x$ decreases from 1 toward $0, f$ is strictly increasing as well as continuous, and $f$ increases from 0 toward $\infty$; that is, to each $y, 0 \leqq y<\infty$, corresponds one and only one $x$ with $0<x \leqq 1, y=f(x)$. For $0 \leqq y<\infty$ we may therefore define $\phi(y)$ by the conditions 


$$
\phi(0)=16, \quad \phi[f(x)]=16 x^{-1 / 2} \quad \text { for } 0<x \leqq 1 .
$$

Clearly, $\phi$ is continuous and strictly increasing on the infinite interval $0 \leqq y<\infty$, with $\int_{1} \phi: f<\infty$. On the other hand, from (a) we have for each $n$

$$
\begin{aligned}
\phi\left[\int_{a_{n+1}}^{a_{n}} f /\left(a_{n}-a_{n+1}\right)\right]\left(a_{n}-a_{n+1}\right)>\phi\left[f\left(m_{n}\right)\right]\left(a_{n}-a_{n+1}\right) \\
\quad=16 m_{n}^{-1 / 2}\left(a_{n}-a_{n+1}\right)=16 a_{n}^{-1}\left(a_{n}-a_{n}^{4}\right) \\
\quad=16\left(1-a_{n}^{3}\right)>16\left(1-a_{n}\right) \geqq 16(15 / 16)=15,
\end{aligned}
$$

which implies

$$
\lim _{\delta \rightarrow 0} \sup _{R \in \overline{\mathbf{r}}_{\delta}(I)} \sum_{B \in F} \phi\left[\int_{B} f /|B|\right]|B|=\infty ;
$$

and from (b)

$$
\phi[f(x)]=16 x^{-1 / 2}=\left(4 x^{-1 / 4}\right)^{2}>[f(x)]^{2} \quad \text { for } 0<x \leqq 1,
$$

which implies

$$
\lim _{y \rightarrow \infty} \phi(y) / y=\infty .
$$

Finally, we may point out that no real generality is added to Theorem (5.13), when diam $E<\infty$ and $\phi \in C((-\infty, \infty))$, by assuming only deferred application of the dominance of $|\phi|$ by $\psi$ or of the convexity of the dominant function $\psi$. For, if the conditions

$$
\begin{gathered}
\phi \in C((-\infty, \infty)), \quad|\phi(y)| \leqq \psi(y) \quad \text { for } 0<M \leqq|y|<\infty, \\
\psi \text { convex on }(-\infty, \infty), \quad \int_{E} \psi: f<\infty
\end{gathered}
$$

are satisfied, let $\alpha=\max _{|y| \leqq M}|\phi(y)|$. Then $\psi_{1}(y)=\psi(y)+\alpha$ is convex on $(-\infty, \infty),|\phi|$ is $\leqq \psi_{1}$ on $(-\infty, \infty)$ and $\int_{E} \psi_{1}: f=\int_{E} \psi: f+\int_{E} \alpha<\infty$. Again, if the conditions

$$
\begin{gathered}
\phi \in C((-\infty, \infty)), \quad|\phi(y)| \leqq \psi(y) \text { on }(-\infty, \infty), \quad \psi \in C((-\infty, \infty)), \\
\psi \text { convex for }-\infty<y \leqq M \text { and for } M \leqq y<\infty, \quad \int_{E} \psi: f<\infty
\end{gathered}
$$

are fulfilled, let $\alpha=\max _{|y| \leqq M}|\psi(y)|$. Then $\psi_{1}(y)=\max [\psi(y), \alpha]$ is convex, and $|\phi|$ is $\leqq \psi_{1}$, on $(-\infty, \infty)$. If $\zeta(y)=\psi_{1}(y)-\psi(y)$ on $(-\infty, \infty)$, it is easily seen that $\zeta \in U C((-\infty, \infty))$, whence $f \in L_{\zeta}(E)$ by Theorem (5.2); thus $\int_{E} \psi_{1}: f=\int_{E} \psi: f-\int_{E} \zeta: f$ exists.

9. The essential nature of the hypothesis of continuity on $\phi$. The extent to which this hypothesis is essential to the result embodied in Theorem (5.12) is rather clearly indicated by the following theorem. 
(9.1) Theorem. If $\phi$ is a function on $(-\infty, \infty)$ and $f \in B L_{\phi}(I)$ implies

$$
\lim _{n \rightarrow \infty}\left(1 / 2^{n}\right) \sum_{j=1}^{2^{n}} \phi\left[2^{n} \int_{(j-1) / 2^{n}}^{j / 2^{n}} f\right]=\int_{I} \phi: f,
$$

then $\phi(y)$ is continuous on $(-\infty, \infty)$.

To prove this theorem we shall show that the assumption of the hypothesis and the contrary of the conclusion leads to a contradiction. For simplicity we first consider the case in which $\phi(y)$ has an hypothetical discontinuity at $y=0$, with

$$
\phi(-1)=\phi(0)=\phi(1)=0, \quad \limsup _{y \rightarrow 0+} \phi(y)>\alpha>0 .
$$

We make these assumptions, and with the object of constructing a function $f \in B L_{\phi}(I)$ which will yield a contradiction we proceed presently to establish a lemma. For this purpose the following notation will be convenient:

$$
\begin{aligned}
\{j, n\} & =\underset{x}{E}\left[(j-1) / 2^{n} \leqq x \leqq j / 2^{n}\right] \quad\left(n=1,2,3, \cdots ; j=1,2, \cdots, 2^{n}\right), \\
S_{n}(f) & =\left(1 / 2^{n}\right) \sum_{j=1}^{2^{n}} \phi\left[2^{n} \int_{\{j, n\}} f\right], \\
P(f) & =\underset{x}{E}[|f(x)|>0] .
\end{aligned}
$$

We also define certain sequences of functions as follows. For $n \geqq 1$, $0<\xi<1$, and $A \subset I$ let $h_{n, \xi}$ be defined on $I$ by

$$
h_{n, \xi}(x)=\left\{\begin{array}{rl}
1 & \text { for } j / 2^{n}-\xi / 2^{n} \leqq x \leqq j / 2^{n} \\
-1 & \text { for } 3 / 4-\xi / 4 \leqq x \leqq 3 / 4+\xi / 4 \\
0 & \text { otherwise }
\end{array} \quad\left(j=1,2, \cdots, 2^{n-1}\right),\right.
$$

let $H_{n, \xi}$ be defined on $(-\infty, \infty)$ to be periodic of period $1 / 2^{n}$ with

$$
H_{n, \xi}\left(x / 2^{n}\right)=h_{n, \xi}(x) \quad \text { for } x \in I \text {; }
$$

let $\delta_{n, A}$ be defined on $I$ by

$$
\delta_{n, A}(x)=\left\{\begin{array}{ll}
0 & \text { for } x \in\{j, n\} \\
1 & \text { otherwise }
\end{array} \text { if } A\{j, n\} \neq 0 \quad\left(j=1,2, \cdots, 2^{n}\right),\right.
$$

and let $g_{n, \xi, A}$ be defined on $I$ by

$$
g_{n, \xi, A}(x)=\delta_{n, A}(x) H_{n, \xi}(x) \quad \text { for } x \in I,
$$

so that $P\left(g_{n, \xi, A}\right)$ is a closed set. These functions being defined, we assert

LEMmA 1. If $A$ is a closed subset of $I$ and $0<\xi<1$ we have

(i) $g_{n, \xi, A}(x)=0$ for $x \in\{j, n\}$ if $A\{j, n\} \neq 0\left(n=1,2,3, \cdots ; j=1,2, \cdots, 2^{n}\right)$; 
(ii) for integers $n_{1}, n_{2}$ with $n_{2} \geqq n_{1} \geqq 1$,

$$
\int_{\left(j, n_{1}\right)} g_{n_{2}, \xi, A}(x) d x=0 \quad\left(j=1,2, \cdots, 2^{n_{1}}\right) ;
$$

(iii) (the hypothesis of Theorem 8 being assumed)

$$
\lim _{n \rightarrow \infty} S_{2 n}\left(g_{n, \xi, A}\right)=(1-|A|) \phi(\xi) / 2 .
$$

Conclusion (i) is immediate from the definition of $\delta_{n, A}$. To prove the other conclusions we regard $A$ and $\xi$ as fixed, drop these letters as subscripts, set

$$
\delta_{n}\left(\left[(j-1) / 2^{n}+j / 2^{n}\right] / 2\right)=\mu_{n, j} \quad\left(n=1,2,3, \cdots ; j=1,2, \cdots, 2^{n}\right),
$$

and proceed as follows. From the definition of $h_{n}$ we have

$$
\int_{I} h_{n}=0 \quad(n=1,2,3, \cdots) \text {; }
$$

whence, for such $n$ and $j=1,2, \cdots, 2^{n}$,

$$
\begin{aligned}
\int_{\{j, n\}} g_{n}(x) d x & =\mu_{n, j} \int_{\{j, n\}} H_{n}(x) d x=\mu_{n, j} \int_{\{1, n\}} H_{n}(x) d x \\
& =\frac{\mu_{n, j}}{2^{n}} \int_{I} H_{n}\left(x / 2^{n}\right) d x=\frac{\mu_{n, j}}{2^{n}} \int_{I} h_{n}(x) d x=0 .
\end{aligned}
$$

For $n_{2} \geqq n_{1} \geqq 1$ we then have

$$
\int_{\left\{j, n_{1}\right\}} g_{n_{2}}=\sum_{\nu=(j-1) 2^{n_{2}-n_{1}}+1}^{j 2^{n_{2}-n_{1}}} \int_{\left\{v, n_{2}\right\}} g_{n_{2}}=0 \quad\left(j=1,2, \cdots, 2^{n_{1}}\right),
$$

and (ii) is proved.

As for (iii) we observe first

$$
\begin{aligned}
\lim _{n \rightarrow \infty} S_{n}\left(h_{n}\right) & =\lim _{n \rightarrow \infty}\left(1 / 2^{n}\right) \sum_{j=1}^{2^{n}} \phi\left[2^{n} \int_{\{j, n\}} h_{n}\right] \\
& =\lim _{n \rightarrow \infty}\left(1 / 2^{n}\right)\left\{\sum_{j=1}^{2 n-1}+\sum_{j=2^{n-1}+1}^{2^{n}}\right\} \phi\left[2^{n} \int_{\{j, n\}} h_{n}\right] \\
& =\left(1 / 2^{n}\right) \phi(\xi) 2^{n-1}+\int_{1 / 2}^{1} \phi: h_{n}=\phi(\xi) / 2,
\end{aligned}
$$

by applying the hypothesis of Theorem (9.1) to the function on $I$ which is zero for $x \in[0,1 / 2]$ and equal to $h_{n}$ for $x \in[1 / 2,1]$. Next we note, for $\nu=1,2, \cdots, 2^{n}$, 


$$
\begin{aligned}
& \sum_{j=(\nu-1) 2^{n}+1}^{\nu 2^{n}} \phi\left[2^{2 n} \int_{\{j, 2 n\}} g_{n}(x) d x\right]=\sum_{j=(\nu-1) 2^{n}+1}^{\nu 2^{n}} \phi\left[2^{2 n} \int_{\{j, 2 n\}} \delta_{n}(x) H_{n}(x) d x\right] \\
& =\mu_{n, \nu} \sum_{j=(\nu-1) 2^{n}+1}^{\nu 2^{n}} \phi\left[2^{2 n} \int_{\{j, 2 n\}} H_{n}(x) d x\right]=\mu_{n, \nu} \sum_{j=1}^{2^{n}} \phi\left[2^{2 n} \int_{\{j, 2 n\}} H_{n}(x) d x\right] \\
& =\mu_{n, \nu} \sum_{j=1}^{2^{n}} \phi\left[2^{n} \int_{\{j, n\}} H_{n}\left(x / 2^{n}\right) d x\right]=\mu_{n, \nu} \sum_{j=1}^{2^{n}} \phi\left[2^{n} \int_{\{j, n\}} h_{n}(x) d x\right] \\
& =\mu_{n, \nu} 2^{n} S_{n}\left(h_{n}\right) .
\end{aligned}
$$

Hence for integral $n \geqq 1$, we have

$$
\begin{aligned}
S_{2 n}\left(g_{n}\right) & =\left(1 / 2^{2 n}\right) \sum_{j=1}^{22 n} \phi\left[2^{2 n} \int_{\{j, 2 n\}} g_{n}(x) d x\right] \\
& =\left(1 / 2^{2 n}\right) \sum_{\nu=1}^{2^{n}} \sum_{j=(\nu-1) 2^{n}+1}^{2^{n}} \phi\left[2^{2 n} \int_{\{j, 2 n\}} g_{n}(x) d x\right] \\
& =\left(1 / 2^{2 n}\right) \sum_{\nu=1}^{2^{n}} \mu_{n, \nu} 2^{n} S_{n}\left(h_{n}\right)=S_{n}\left(h_{n}\right) \sum_{\nu=1}^{2^{n}} \mu_{n, \nu} / 2^{n}=S_{n}\left(h_{n}\right) \int_{I} \delta_{n}(x) d x .
\end{aligned}
$$

From the fact that $I-A$ is an open set, the density of the set of points $j / 2^{n}\left(n=1,2,3, \cdots ; j=0,1, \cdots, 2^{n}\right)$ in $I$, and the definition of $\delta_{n}$ one may infer $\lim _{n \rightarrow \infty} \int_{I} \delta_{n}(x) d x=1-|A|$. Using also (b) one obtains conclusion (iii).

Returning now to the proof of Theorem (9.1), let $\eta$ be an arbitrary number satisfying the inequality $0<\eta<1$, and for each integer $n>0$ let $\xi_{n}$ be a number satisfying the conditions $0<\xi_{n}<\eta / 2^{n}, \phi\left(\xi_{n}\right)>\alpha$.

We are ready to begin an inductive definition of a sequence of functions $f_{n}$ $(n=1,2,3, \cdots)$. Let $A_{0}$ be the vacuous set; and let $N_{1} \geqq 1$ be so large that, upon defining $f_{1}=g_{N_{1}}, \xi_{1}, A_{0}$, we have in accordance with Lemma 1 (iii),

$$
S_{2 N_{1}}\left(f_{1}\right) \geqq \phi\left(\xi_{1}\right) / 2-1 / 2 \text {. }
$$

For the second stage of the procedure let $A_{1}=P\left(f_{1}\right)$; and let $N_{2}>2 N_{1}$ be so large that, upon defining $f_{2}=g_{N_{2}, \xi_{2}, \Delta_{1}}$, we have in accordance with Lemma 1 (iii) and the hypothesis of Theorem (9.1),

We observe $\left|A_{1}\right|=\xi_{1}$.

$$
\begin{gathered}
S_{2 N_{2}}\left(f_{2}\right) \geqq\left(1-\left|A_{1}\right|\right) \phi\left(\xi_{2}\right) / 2-1 / 2^{2}, \\
\left|S_{2 N_{2}}\left(f_{1}\right)\right| \leqq 1 / 2^{2} .
\end{gathered}
$$

For the third stage, let $A_{2}=P\left(f_{1}\right)+P\left(f_{2}\right)=A_{1}+P\left(f_{2}\right)$; and let $N_{3}>2 N_{2}$ be so large that, upon defining $f_{3}=g_{N_{3}}, \xi_{3}, A_{2}$, we have in accordance with Lemma 1 (iii) and the hypothesis of Theorem (9.1),

$$
\begin{gathered}
S_{2 N_{8}}\left(f_{3}\right) \geqq\left(1-\left|A_{2}\right|\right) \phi\left(\xi_{3}\right) / 2-1 / 2^{3}, \\
\left|S_{2 N_{8}}\left(f_{1}+f_{2}\right)\right| \leqq 1 / 2^{3} .
\end{gathered}
$$


The inequality $\left|A_{2}\right|<\xi_{1}+\xi_{2}$ should be noted. In general, $f_{j}(j=1,2, \cdots, n-1)$ having been defined, let $A_{n-1}=A_{n-2}+P\left(f_{n-1}\right)$; and let $N_{n}>2 N_{n-1}$ be so large that upon defining $f_{n}=f_{N_{n}, \xi_{n}, A_{n-1}}$, we have

(c)

$$
\begin{gathered}
S_{2 N_{n}}\left(f_{n}\right) \geqq\left(1-\left|A_{n-1}\right|\right) \phi\left(\xi_{n}\right) / 2-1 / 2^{n}, \\
\left|S_{2 N_{n}}\left(f_{1}+f_{2}+\cdots+f_{n-1}\right)\right| \leqq 1 / 2^{n} .
\end{gathered}
$$

We note $\left|A_{n-1}\right|<\xi_{1}+\xi_{2}+\cdots+\xi_{n-1}$.

Finally we define

$$
F(x)=\sum_{j=1}^{\infty} f_{j}(x) \quad \text { for } x \in I, \quad A=\sum_{j=1}^{\infty} A_{j},
$$

observing that $P(f)=A$ and that $A_{j} \subset A_{j+1}(j=1,2,3, \cdots)$ implies

$$
|A|=\lim _{j \rightarrow \infty}\left|A_{j}\right| \leqq \sum_{j=1}^{\infty} \xi_{j}<\eta
$$

For $n \geqq 2$ we may write

and obtain

$$
F=\sum_{\nu=1}^{n-1} f_{\nu}+f_{n}+\sum_{\nu=n+1}^{\infty} f_{\nu}
$$

(d)

$$
S_{2 N_{n}}(F)=S_{2 N_{n}}\left(\sum_{\nu=1}^{n-1} f_{\nu}\right)+S_{2 N_{n}}\left(f_{n}\right) \text {. }
$$

This arises from the fact that for each $j\left(j=1,2, \cdots, 2^{2 N_{n}}\right)$ we have by Lemma 1 (ii)

$$
\int_{\left\{j, 2 N_{n}\right\}}\left(\sum_{\nu=n+1}^{\infty} f_{\nu}\right)=\sum_{\nu=n+1}^{\infty} \int_{\left\{j, 2 N_{n}\right\}} f_{\nu}=0,
$$

and by Lemma 1 (i)

$$
\left[\int_{\left\{j, 2 N_{n}\right\}}\left(\sum_{\nu=1}^{n-1} f_{\nu}\right)\right]\left[\int_{\left\{j, 2 N_{n}\right\}} f_{n}\right]=0
$$

which together imply

$$
\begin{aligned}
& \phi\left[2^{2 N_{n}} \int_{\left\{j, 2 N_{n}\right\}} F\right]=\phi {\left[2^{2 N_{n}} \int_{\left\{j, 2 N_{n}\right\}}\left(\sum_{\nu=1}^{n-1} f_{\nu}\right)+2^{2 N_{n}} \int_{\left\{j, 2 N_{n}\right\}} f_{n}\right] } \\
&=\phi\left[2^{2 N_{n}} \int_{\left\{j, 2 N_{n}\right\}}\left(\sum_{\nu=1}^{n-1} f_{\nu}\right)\right]+\phi\left[2^{2 N_{n}} \int_{\left\{j, 2 N_{n}\right\}} f_{n}\right] .
\end{aligned}
$$

From (c) and (d) we have

$$
S_{2 N_{n}}(F) \geqq\left(1-\left|A_{n-1}\right|\right) \phi\left(\xi_{n}\right) / 2-1 / 2^{n-1} \geqq\left(1-\left|A_{n-1}\right|\right) \alpha / 2-1 / 2^{n-1} \text {, }
$$


whence

$$
\lim _{n \rightarrow \infty} S_{2 N_{n}}(F) \geqq(1-|A|) \alpha / 2>(1-\eta) \alpha / 2 .
$$

But from the hypothesis of Theorem (9.1) we obtain

$$
\lim _{n \rightarrow \infty} S_{n}(F)=\int_{I} \phi: F=0,
$$

an obvious contradiction. This completes the proof of Theorem (9.1) in the case characterized by conditions (a).

We shall now show how a proof of Theorem (9.1) in the general case can be obtained from the particular case already considered. Let $\beta$ stand for the class of functions $f$ each of which is measurable on $I$ and assumes no more than three values. A function $\phi$ defined on $(-\infty, \infty)$ will be said to possess property (B) if and only if

$$
f \in \beta \text { implies } \lim _{n \rightarrow \infty}\left(1 / 2^{n}\right) \sum_{n} \phi\left[2^{n} \int_{\{j, n\}} f\right]=\int_{I} \phi: f .
$$

The result obtained above may now be formulated thus:

(i) If $\phi$ has property (B), with $\phi(-1)=\phi(0)=\phi(1)=0$, then lim $\sup _{y \rightarrow 0+} \phi(y)$ is $\leqq 0$.

We assert

(ii) $A$ necessary and sufficient condition that $\phi(y)$ be continuous for all $y$ is that it possess property (B).

The necessity of the condition may be inferred at once from Theorem (5.2). To prove the sufficiency we first verify without difficulty that if $\phi_{1}$ and $\phi_{2}$ have property (B), so also do $\phi_{1}+\phi_{2}$ and $c \phi_{1}(a y+b)$, where $a, b$, and $c$ are arbitrary real numbers. Now let $\phi$ be assumed to have property (B) and to be discontinuous at an arbitrary point $y_{1}$; then $\phi_{1}(y)=\phi\left(y-y_{1}\right)$ has property (B) and is discontinuous at $y=0$. Also, for $-\infty<y<\infty$, let

$$
\phi_{2}(y)=\phi_{1}(-1) y(1-y) / 2+\phi_{1}(0)(y+1)(y-1)+\phi_{1}(1)(-y)(1+y) / 2,
$$

so that $\phi_{2}$, being continuous, has property (B). The function $\phi_{3}=\phi_{1}+\phi_{2}$ then has property $(\mathrm{B})$, with $\phi_{3}(-1)=\phi_{3}(0)=\phi_{3}(1)=0$, and $\phi_{3}$ is discontinuous at $y=0$. Of the four functions $\pm \phi_{3}( \pm y)$, at least one is a function $\phi_{4}$ possessing property $(B)$, with $\phi_{4}(-1)=\phi_{4}(0)=\phi_{4}(1)=0$ and with $\lim \sup _{y \rightarrow 0+} \phi_{4}(y)>0$, in contradiction to (i).

This completes the proof of Theorem (9.1).

REMARK. In connection with Theorem (9.1) it is worth noting that the conditions $\phi \in C((-\infty, \infty)), f \in B L_{\phi}(I)$ imply the existence of a function $\psi$ convex on $(-\infty, \infty)$ and depending upon $f$, with $|\phi(y)| \leqq \psi(y)$ on $(-\infty, \infty)$ and $f \in L_{\psi}(I)$. It would be of some interest to prove or disprove either of the following conjectures. (1) The hypothesis of Theorem (9.1) implies that to 
each $f \in L_{\phi}(I)$ corresponds a function $\psi$ convex on $(-\infty, \infty)$, with $|\phi(y)| \leqq \psi(y)$ on $(-\infty, \infty)$ and $f \in L_{\psi}(I)$. (2) The hypothesis of Theorem (9.1) implies the existence of a function $\psi$ convex on $(-\infty, \infty)$, with $|\phi(y)| \leqq \psi(y)$ on $(-\infty, \infty)$ and $L_{\phi}(I) \subset L_{\psi}(I)$ (that is, $L_{\phi}(I)=L_{\psi}(I)$ ). Attempts which we have made in this direction have met with no success.

10. Concerning the replacement of hypothesis (1.6) by (2.10). In this section we propose to determine as definitely as possible under what circumstances the sample sets need only be of positive measure; that is, the " $k$-hypothesis" (1.6) can be relaxed to (2.10). A basic, though simple, result of this kind is embodied in Theorem (5.1). In RS it has been seen that, in case $E=I$ and $\phi(y)=y$ for all $y$, the question turns entirely on whether $f \in R(I)$, the class of functions Riemann integrable on $I$. Thus it is natural to restrict the considerations of this section to the case in which $E$ is a bounded interval, say $E=I$.

We begin by establishing

(10.1) Theorem. If $f \in L(I), \phi \in C(\Re(f)), \phi: f \in L(I)-R(I)$, and (1.6) is replaced by (2.10),

$$
\lim _{\delta \rightarrow 0} \sum_{B \in F \in \Gamma_{\delta}(I)} \phi\left[\mathfrak{M}_{A B} f\right]|B|
$$

does not exist. Thus, when $\phi: f \in L(I)-R(I)$, the k-hypothesis is indispensable to Theorems (5.2)-(5.13).

Proof. However small $\delta>0$ may be, let $F \in \bar{\Gamma}_{\delta}(I)$. If $\phi: f$ is not essentially bounded on $I$, there is at least one interval $B \in F$ on which $\phi: f$ is not essentially bounded; that is, for arbitrary $M>0$ there exists a measurable set $A \subset B$ with

$$
|A|>0, \quad|\phi[f(x)]|>M \quad \text { for } x \in A .
$$

The set

$$
C=B \underset{x}{E}[f(x) \widetilde{\varepsilon} \Re(f) \text { for } f \text { on } B]
$$

is of measure zero. Hence $A(B-C)$ is of positive measure, and there exist points $x_{1}$ with

$$
\left|\phi\left[f\left(x_{1}\right)\right]\right|>M, \quad x_{1} \in \Re(f) \quad \text { for } f \text { on } B ;
$$

that is, there exists a measurable set $A_{1} \subset B$ with

$$
\left|A_{1}\right|>0, \quad\left|\phi\left[\mathfrak{M}_{A_{1}} f\right]\right|>M .
$$

Thus the sum in question is unbounded.

Secondly, if $\phi: f$ is essentially bounded on $I$ and $B \in F$, let

$$
\underset{x \in B}{\operatorname{ess} \inf } \phi[f(x)]=l, \quad \underset{x \in B}{\operatorname{ess} \sup } \phi[f(x)]=L .
$$


The same reasoning now suffices to show that the sum in question can be made arbitrarily close to either the essential lower or essential upper Darboux sum for $\phi: f$ corresponding to the family of intervals $F$. Hence the $\lim _{\delta \rightarrow 0}$ of the sum does not exist.

We next proceed in the direction of obtaining a favorable result when $f \in R(I)$. Without the $k$-hypothesis, even the conditions $f \in C(I)$ and $\phi \in C(\Re(f))$ are shown to be insufficient for the existence of $\int_{I} \phi: f$, and hence insufficient for (1.9), by the trivial example

$$
f(x)=x \quad \text { for } \quad x \in I, \quad \phi(y)=1 / y \quad \text { for } \quad y \in(0,1)=\Re(f) .
$$

The nature of Theorems (5.8)-(5.12) suggests that we consider assuming

$$
f \in R(I), \quad \phi \in C(\Re(f)), \quad \lim _{\delta \rightarrow 0} \sup _{F \in \mathrm{r}_{\delta}(I)} \sum_{B \in F}\left|\phi\left[\mathfrak{M}_{A B} f\right]\right||B|<\infty .
$$

We assert, however,

(10.3) ThEOREM. The conditions (10.2) are equivalent to the conditions

$$
f \in R(I), \quad \phi \in B C(\Re(f)) \text {. }
$$

Proof. It is evident that this pair of conditions implies the set (10.2). On the other hand, if $\phi \tilde{\boldsymbol{\varepsilon}} B(\Re(f))$, let $a \tilde{\boldsymbol{\varepsilon}} \Re(f)$ be an endpoint of $\Re(f)$ in every neighborhood of which $\phi$ is unbounded; and let $F$ be an arbitrary family $\in \bar{\Gamma}_{\delta}(I)$ for any $\delta>0$. Then at least one interval $B \in F$ has the property that the ess inf, or ess sup, of $f$ on $B$ is $a$. Since $a \tilde{\varepsilon} \Re(f)$, it follows at once that as $A B$ ranges over the subsets of $B$ having measure $>0, \mathfrak{M}_{A B} f$ assumes all values in a unilateral neighborhood of $a$. Hence $\phi\left[\mathfrak{M}_{A B} f\right]|B|$ is an unbounded function of $A B$, and the sum in (10.2) is unbounded for each $\delta>0$.

As a preliminary step in the desired direction we next establish

(10.4) THEOREM. The conditions $f \in R(I)$ and $\phi \in C(\overline{\Re(f)})$ are sufficient for (1.9), even with (1.6) replaced by (2.10).

Proof. We may assume $f$ itself to be an element of $R(I)$, with $f(x) \in \overline{\Re(f)}$ for $x \in I . \overline{\Re(f)}$ is a bounded interval, whence $\phi \in U C(\overline{\Re(f)})$. Thus to $\epsilon>0$ corresponds $\eta=\eta(\epsilon)>0$ such that the conditions

$$
y_{1}, y_{2} \in \overline{\Re(f)},\left|y_{1}-y_{2}\right|<\eta \text { imply }\left|\phi\left(y_{1}\right)-\phi\left(y_{2}\right)\right|<\epsilon / 6 ;
$$

and we then have

$$
\left|\phi\left(y_{1}\right)-\phi\left(y_{2}\right)\right|<\epsilon / 6+\epsilon\left|y_{1}-y_{2}\right| /(6 \eta) \quad \text { for } y_{1}, y_{2} \in \overline{\Re(f)} .
$$

Let $g$ and $h$ be functions continuous on $I$ and satisfying the conditions

$$
\begin{gathered}
g(x) \leqq f(x) \leqq h(x) \quad \text { for } \quad x \in I, \quad \min _{x \in I} g(x) \in \overline{\Re(f)}, \quad \max _{x \in I} g(x) \in \overline{\Re(f)}, \\
\min _{x \in I} h(x) \in \overline{\Re(f)}, \quad \max _{x \in I} h(x) \in \overline{\Re(f)}, \quad \int_{I}(h-g)<\eta .
\end{gathered}
$$


Let $\delta_{1}=\delta_{1}\left(h-g, \phi_{1}, \eta\right)>0$ correspond to $\eta$ for the functions $h-g$ and $\phi_{1}(y)=y$ $(-\infty<y<\infty)$ in accordance with Theorem (5.1); let $\delta_{2}=\delta_{2}(h, \phi, \epsilon / 6)>0$ correspond to $\epsilon / 6$ for the functions $h$ and $\phi$ in accordance with Theorem (5.1); and let $\delta=\min \left[\delta_{1}, \delta_{2}\right]$. For $F \in \Gamma_{\delta}(I)$ we then have

$$
\begin{aligned}
& \sum_{B \in F}\left|\mathfrak{M}_{B} \phi: f-\phi\left[\mathfrak{M}_{A B} f\right]\right||B| \\
& \leqq \sum_{B \in F} \int_{B}|\phi: f-\phi: h|+\sum_{B \in F}\left|\mathfrak{M}_{B} \phi: h-\phi\left[\mathfrak{M}_{A B} h\right]\right||B| \\
& +\sum_{B \in F}\left|\phi\left[\mathfrak{M}_{A B} h\right]-\phi\left[\mathfrak{M}_{A B} f\right]\right||B| \text {. }
\end{aligned}
$$

On the right the first term is

$$
\begin{aligned}
\int_{I}|\phi: f-\phi: h| & <\epsilon / 6+\epsilon \int_{I}|f-h| /(6 \eta) \leqq \epsilon / 6+\int_{I}(h-g) /(6 \eta) \\
& <\epsilon / 6+\epsilon / 6=\epsilon / 3 .
\end{aligned}
$$

By the choice of $\delta\left(\leqq \delta_{2}\right)$ the second term on the right is $<\epsilon / 6$. The third term is not greater than

$$
\begin{aligned}
& \sum_{B \in F}\left\{\epsilon / 6+\epsilon\left|\mathfrak{M}_{A B} h-\mathfrak{M}_{A B} f\right| /(6 \eta)\right\}|B| \\
& \quad \leqq \epsilon / 6+\epsilon /(6 \eta) \sum_{B \in F}\left\{|B| \mathfrak{M}_{A B}(h-g)-\int_{B}(h-g)+\int_{B}(h-g)\right\} \\
& \quad \leqq \epsilon / 6+\epsilon /(6 \eta) \sum_{B \in F}\left|\mathfrak{M}_{A B}(h-g)-\mathfrak{M}_{B}(h-g)\right||B|+\epsilon /(6 \eta) \int_{I}(h-g),
\end{aligned}
$$

which by the choice of $\delta\left(\leqq \delta_{1}\right)$ is less than

$$
\epsilon / 6+[\epsilon /(6 \eta)] \eta+\epsilon / 6=\epsilon / 2 \text {. }
$$

The proof is now complete.

For use in demonstrating the next theorem the following lemma will be convenient.

(10.5) Lemma. Let $f \in R(I), \phi \in B C(\Re(f)){\text {, } \sup _{\mathrm{y}} \in \Re(f)}|\phi(y)|<M<\infty$,

$$
\begin{aligned}
& 0<\eta<(\beta-\alpha) / 2, \text { and } \\
& \alpha \tilde{\boldsymbol{\varepsilon}} \Re(f) \quad[\beta \tilde{\boldsymbol{\varepsilon}} \Re(f)], \\
& E_{\eta}=\underset{x}{E}[f(x) \leqq \alpha+\eta] \quad\left[E_{\eta}=\underset{x}{E}[f(x) \geqq \beta-\eta]\right] .
\end{aligned}
$$$$
\alpha=\inf _{x \in I} f(x), \quad \beta=\sup _{x \in I} f(x), \quad \beta-\alpha>0,
$$

To $\epsilon>0$ corresponds $\eta=\eta(\epsilon)>0$ and $\delta=\delta(\eta)>0$ such that if $F \in \Gamma_{\delta}(I)$ and $F_{1}$ 
represents the subfamily of $F$ in which each set $B$ has the property $\left|B E_{\eta}\right|>0$, we have $\sum_{B \in F_{1}}|B|<\epsilon / M$, whence

$$
\sum_{B \in F_{1}}\left|\mathfrak{M}_{B} \phi: f\right||B|<\epsilon \text { and } \sum_{B \in F_{1}}\left|\phi\left[\mathfrak{M}_{A B} f\right]\right||B|<\epsilon .
$$

Proof. We have $0=\left|E_{x}[f(x)=\alpha]\right|=\lim _{\eta \rightarrow 0}\left|E_{\eta}\right| ;$ let $\eta$ satisfy the conditions

$$
0<\eta<(\beta-\alpha) / 2, \quad\left|E_{\eta}\right|<\epsilon / M \text {. }
$$

We may assume $f$ itself to be an element of $R(I)$. Any point $x \in \bar{E}_{\eta}-E_{\eta}$, where $\bar{E}_{\eta}$ represents the closure of $E_{\eta}$, is then a point of discontinuity of $f$; and since the discontinuities of $f$ are a set of measure zero, we have $\left|\bar{E}_{\eta}\right|$ $=\left|E_{\eta}\right|<\epsilon / M$. Let

$$
I-\bar{E}_{\eta}=\sum_{i} O_{i}
$$

where $\sum_{i}\left\{O_{i}\right\}$ is a countable set of disjoint open intervals. Let $k$ be a finite number of these intervals with the property

$$
\sum_{i=1}^{k}\left|O_{i}\right|>1-\epsilon / M
$$

From each end of each $O_{i}(i=1,2, \cdots, k)$ delete a semi-open interval to leave an open interval $O_{i}^{\prime}$, in such manner that we have

$$
\sum_{i=1}^{k}\left|O_{i}^{\prime}\right|>1-\epsilon / M
$$

and let the measure of the smallest deleted interval be $\delta(\delta>0)$. For $F \in \Gamma_{\delta}(I)$ and $F_{2}=F-F_{1}$ we then have

$$
\begin{gathered}
\sum_{B \in F_{2}}|B| \geqq \sum_{i=1}^{k}\left|O_{i}^{\prime}\right|>1-\epsilon / M, \quad \sum_{B \in F_{1}}|B|<\epsilon / M, \\
\sum_{B \in F_{1}}\left|\mathfrak{M}_{B} \phi: f\right||B|<M \epsilon / M=\epsilon, \quad \sum_{B \in F_{1}}\left|\phi\left[\mathfrak{M}_{A B} f\right]\right||B| \leqq M \epsilon / M=\epsilon .
\end{gathered}
$$

(10.6) THEOREM. The conditions $f \in R(I)$ and $\phi \in B C(\Re(f))$ are sufficient for (1.9), even with (1.6) replaced by (2.10).

Proof. Let $f$ itself be an element of $R(I)$. If $\Re(f)=\overline{\Re(f)}$, the conclusion follows from Theorem (10.4). If $\alpha=\inf _{x \in I} f(x), \beta=\sup _{x \in I} f(x), \beta-\alpha>0$, $\alpha \tilde{\varepsilon} \Re(f)[\beta \widetilde{\varepsilon} \Re(f)]$, and $0<\eta<(\beta-\alpha) / 2$, let

$$
\begin{array}{ll}
f_{\eta}(x)=\alpha+\eta & \text { for } x \in \underset{x}{E}[f(x)<\alpha+\eta] \\
{\left[f_{\eta}(x)=\beta-\eta\right.} & \text { for } x \in \underset{x}{E}[f(x)>\beta-\eta]] ;
\end{array}
$$

and for other values of $x$ let $f_{\eta}(x)=f(x)$. One easily sees

$$
f_{\eta} \in R(I), \quad \Re\left(f_{\eta}\right)=\overline{\Re\left(f_{\eta}\right)} \subset \Re(f), \quad \phi \in C \overline{\left(\overline{\Re\left(f_{\eta}\right)}\right) .}
$$


Let $\eta$ and $\delta_{1}>0$ correspond to $\epsilon / 9$ in accordance with Lemma (10.5); let $\delta_{2}=\delta_{2}\left(f_{\eta}, \phi, \epsilon / 9\right)>0$ correspond to $\epsilon / 9$ for the functions $f_{\eta}$ and $\phi$ in accordance with Theorem (10.4); and let $\delta=\min \left[\delta_{1}, \delta_{2}\right]$. For $F \in \Gamma_{\delta}(I)$ we then have

$$
\begin{aligned}
& \sum_{B \in F}\left|\mathfrak{M}_{B} \phi: f-\phi\left[\mathfrak{M}_{A B} f\right]\right||B| \\
& \leqq \\
& \quad \sum_{B \in F}\left|\mathfrak{M}_{B} \phi: f-\mathfrak{M}_{B} \phi: f_{\eta}\right||B|+\sum_{B \in F}\left|\mathfrak{M}_{B} \phi: f_{\eta}-\phi\left[\mathfrak{M}_{A B} f_{\eta}\right]\right||B| \\
& \quad+\sum_{B \in F}\left|\phi\left[\mathfrak{M}_{A B} f_{\eta}\right]-\phi\left[\mathfrak{M}_{A B}\right]\right||| B \mid \\
& <\sum_{B \in F_{1}}\left|\mathfrak{M}_{B} \phi: f\right||B|+\sum_{B \in F_{1}}\left|\mathfrak{M}_{B} \phi: f_{\eta}\right||B|+\epsilon / 9 \\
& \quad+\sum_{B \in F_{1}}\left|\phi\left[\mathfrak{M}_{A B} f_{\eta}\right]\right||B|+\sum_{B \in F_{1}}\left|\phi\left[\mathfrak{M}_{A B} f\right]\right||B|<\epsilon,
\end{aligned}
$$

where $F_{1}$ has the significance attached to it in Lemma (10.5). ditions

The question of whether the $k$-hypothesis can be relaxed under the con-

$$
f \in L(I)-R(I), \quad \phi: f \in R(I), \quad \phi \in U C \overline{(\Re(f)})
$$

is still open. That it sometimes can is illustrated by the trivial example

$$
f \in L(I), \quad \phi(y)=\text { const. } \quad \text { for }-\infty<y<\infty .
$$

That it sometimes cannot, even if $\phi$ is a very simple convex function and $f$ is bounded, may be shown as follows. Let $\phi(y)=|y|$ for $-1 \leqq y \leqq 1$; let $E \subset I$ be a non-dense perfect set, with $|E|=1 / 2$, and let $f(x)=1$ for $x \in E, f(x)=-1$ for $x \in I-E$. For each $m(m=1,2,3, \cdots)$ let $F_{m} \in \Gamma_{1}(I)$ be the family of $2^{m}$ equal subintervals of $I$. In each one of these intervals there exists a subset of $I-E$ of measure $>0$. In each one of at least half of these intervals there exists a subset of $E$ of measure $>0$, and hence a subset $A B$ with $|A B|>0$, such that $\mathfrak{M}_{A B} f=\int_{A B} f=0$. In any one of the remaining subintervals the maximum absolute value of the integral mean of $f$ on any subset is 1 . Hence we have

$$
\underset{m \rightarrow \infty}{\liminf } \sum_{B \in F_{m}} \phi\left[\mathfrak{M}_{A B} f\right]|B| \leqq 1 / 2<1=\int_{I} \phi: f .
$$

This example being in hand, some interest may attach to

(10.7) THEOREM. The conditions $f \in L(I), \phi: f \in R(I)$, $\phi$ convex on $\Re(f)$, and (1.6) replaced by (2.10), imply

$$
\lim _{\delta \rightarrow 0} \sup _{F \in \Gamma_{\delta}(I)} \phi\left[\mathfrak{M}_{A B} f\right]|B| \leqq \int_{I} \phi: f<\infty .
$$

Proof. We may assume $\phi: f$ itself to be an element of $R(I)$. Let $\epsilon$ be an arbitrary positive number and let $g$ be a function continuous on $I$ and satisfying the conditions 


$$
\phi[f(x)] \leqq g(x) \quad \text { for } \quad x \in I, \quad \int_{I}(g-\phi: f)<\epsilon / 2 .
$$

In accordance with Theorem (5.1) let $\delta=\delta\left(g, \phi_{1}, \epsilon / 2\right)>0$ correspond to $\epsilon / 2$ for the functions $g$ and $\phi_{1}(y)=y(-\infty<y<\infty)$. Using Jensen's inequality we then have, for $F \in \Gamma_{\delta}(I)$,

$$
\begin{aligned}
\sum_{B \in F} \phi\left[\mathfrak{M}_{A B} f\right]|B| & \leqq \sum_{B \in F}\left[\mathfrak{M}_{A B} \phi: f\right]|B| \leqq \sum_{B \in F}\left[\mathfrak{M}_{A B} g\right]|B| \\
& <\int_{I} g+\epsilon / 2<\int_{I} \phi: f+\epsilon .
\end{aligned}
$$

In the example last cited above $f$ is in the class $L(I)-R(I)$ because of the fact that, though bounded, it has discontinuities at a set of positive measure. This gives rise to the question: do there exist examples in which it is impossible to relax the $k$-hypothesis when $f \in L(I)-R(I)$ because $f$ is unbounded, even though $f$ is improperly Riemann integrable on $I, \phi: f \in R(I)$, and (1.9) holds with the $k$-hypothesis in force? Should no such example exist, it would probably be possible to enlarge the class of cases already determined by Theorem (10.6) in which the $k$-hypothesis can be relaxed by obtaining an affirmative answer to some such question as the following. Do the conditions

$$
\left.f \in R^{*}(I), \quad \phi: f \in R(I), \quad \phi \in U C \overline{(\Re(f)}\right)
$$

imply that the $k$-hypothesis can be relaxed?

The answer to this question, however, is negative, as one may see from the ensuing example. Let

$$
\begin{aligned}
& \phi(y)= \begin{cases}0 \text { for } y=2\left(2^{n}-1\right) & (n=0,1,2, \cdots) ; \\
2^{n} \text { for } y=2\left(2^{n}-1\right)-2^{n-1} & (n=1,2,3, \cdots) ; \\
\text { linear between consecutive values of } y \text { for which } \phi \text { is already defined; }\end{cases} \\
& f(x)= \begin{cases}0 \text { for } 1 / 2^{2}<x \leqq 1, \quad x=0 ; \\
2\left(2^{n}-1\right) \text { for } 1 /\left[4\left(2^{n+1}-1\right)^{2}\right]<x \leqq 1 /\left[4\left(2^{n}-1\right)^{2}\right]\end{cases} \\
& \quad(n=1,2,3, \cdots) .
\end{aligned}
$$

Then we have $f \in R^{*}(I)$, since it is a non-negative function dominated on $I$ by $f_{1}$ where $f_{1}(x)=1 / x^{1 / 2}$ for $0<x \leqq 1, f_{1}(0)=0 ; \phi: f \in R(I)$, because it vanishes identically on $I ; \Re(f)=\overline{\Re(f)}=E_{y}[0 \leqq y<\infty]$; and $\phi \in U C(\overline{\Re(f)})$, for its graph consists of the upper sides of an infinite sequence of similar triangles all with bases on the $y$-axis. If now $F \in \bar{\Gamma}_{1}(I)$ is the family of $2^{n}$ equal subintervals of $I$, the terms of the sum

$$
\sum_{B \in F} \phi\left[\mathfrak{M}_{A B} f\right]|B|
$$


are all non-negative and the value of the term containing the interval $B=\left[0,1 / 2^{n}\right]$ can be made arbitrarily large by a suitable choice of $A$. Thus the $\lim _{\delta \rightarrow 0}$ of the $\sup _{F \in \Gamma_{\delta(I)}}$ of such sums is infinite.

In the foregoing example $\phi$ is unbounded. It is natural therefore to ask if the situation is the same when $\phi$ is bounded; in answer we have theorems as follows.

(10.8) THEOREM. The conditions $f \in R^{*}(I)$ and $\phi \in B U C(\Re(f))$ are sufficient for (1.9), even with (1.6) replaced by (2.10).

Proof. To $\epsilon>0$ corresponds $\eta=\eta(\epsilon)>0$ such that the conditions

$$
y_{1}, y_{2} \in \Re(f),\left|y_{1}-y_{2}\right|<\eta \quad \text { imply }\left|\phi\left(y_{1}\right)-\phi\left(y_{2}\right)\right|<\epsilon / 16,
$$

whence

$$
\left|\phi\left(y_{1}\right)-\phi\left(y_{2}\right)\right|<\epsilon / 16+\epsilon\left|y_{1}-y_{2}\right| /(16 \eta) \quad \text { for } y_{1}, y_{2} \in \Re(f) .
$$

Let $M$ satisfy the condition $|\phi(y)|<M<\infty$ for $y \in \Re(f)$.

Let $D \subset I$ be the set of points defined by the condition $x \in D$ if and only if ess $\lim \sup _{x^{\prime} \rightarrow x}\left|f\left(x^{\prime}\right)\right|=\infty$. Clearly $D$ is closed, with $|D|=0$. Let the open set $I-D$ be constituted of the disjoint open intervals $O_{i}(i=1,2,3, \cdots)$, and let $m$ be such that $\sum_{i=1}^{m}\left|O_{i}\right|>1-\epsilon /(8 M)$. From each end of $O_{i}$ $(i=1,2,3, \cdots, m)$ delete an open interval of length $\epsilon /(16 \mathrm{Mm})$ and designate the remaining closed interval by $F_{i}$, so that $\sum_{i=1}^{m}\left|F_{i}\right|>1-\epsilon /(4 M)$. On each interval $F_{i}$ the function $f$ is essentially bounded. Let $F_{N}$ be the truncated function $f$ specified in the definition of the condition $f \in R^{*}(I)$ as given in $\$ 2$, $N$ being chosen to satisfy the conditions

$$
\int_{I}\left|f-f_{N}\right|<\eta, \quad \underset{x \in F_{i}}{\operatorname{ess} \sup _{i}}|f(x)|<N<\infty \quad(i=1,2, \cdots, m) .
$$

Finally, let $\delta_{1}=\delta_{1}(f, \phi, \epsilon / 8)>0$ correspond to $\epsilon / 8$ for the functions $f_{N}$ and $\phi$ in accordance with Theorem (10.6); let $\delta_{2}=\epsilon /(16 \mathrm{Mm})$; and let $\delta=\min \left[\delta_{1}, \delta_{2}\right]$. For convenience let $F_{i}^{\prime}$ represent the closed interval obtained from $F_{\mathfrak{i}}$ $(i=1,2, \cdots, m)$ by deleting from each end of $F_{i}$ a semi-open interval of length $\epsilon /(16 M m)$, so that we have $\sum_{i=1}^{m}\left|F_{i}^{\prime}\right|>1-3 \epsilon /(8 M)$ and $\left|I-\sum_{i=1}^{m} F_{i}^{\prime}\right|$ $=1-\sum_{i=1}^{m}\left|F_{i}^{\prime}\right|<3 \epsilon /(8 M)$. For $F \in \Gamma_{\delta}(I)$ let $F_{1}$ represent the subfamily of $F$ defined by the condition $B \in F_{1}$ if and only if $B \sum_{i=1}^{m} F_{i}^{\prime} \neq 0$; and let $F_{2}=F-F_{1}$. For $F \in \Gamma_{\delta}(I)$ we than have

$$
\begin{aligned}
& \sum_{B \in F}\left|\mathfrak{M}_{B} \phi: f-\phi\left[\mathfrak{M}_{A B} f\right]\right||B| \\
& \leqq \int_{I}\left|\phi: f-\phi: f_{N}\right|+\sum_{B \in F}\left|\mathfrak{M}_{B} \phi: f_{N}-\phi\left[\mathfrak{M}_{A B} f_{N}\right]\right||B| \\
& +\sum_{B \in F}\left|\phi\left[\mathfrak{M}_{A B} f_{N}\right]-\phi\left[\mathfrak{M}_{A B} f\right]\right||B| \text {. }
\end{aligned}
$$


On the right the first term is less than

$$
\epsilon / 16+\epsilon \int_{I}\left|f-f_{N}\right| /(16 \eta)<\epsilon / 16+\epsilon / 16=\epsilon / 8 .
$$

By our choice of $\delta\left(\leqq \delta_{1}\right)$ the second term on the right is $<\epsilon / 8$. The third term may be written

$$
\left\{\sum_{B \in F_{1}}+\sum_{B \in F_{2}}\right\}\left|\phi\left[\mathfrak{M}_{A B} f_{N}\right]-\phi\left[\mathfrak{M}_{A B} f\right]\right||B| .
$$

Since $\delta$ is $\leqq \delta_{2}$, each set $B \in F_{1}$ contains only points of $\sum_{i=1}^{m} F_{i}$; whence, by choice of $N$ and $f_{N}$,

$$
\sum_{B \in F_{1}}\left|\phi\left[\mathfrak{M}_{A B} f_{N}\right]-\phi\left[\mathfrak{M}_{A B} f\right]\right||B|=0 .
$$

As for the remaining sum, from the relation $\sum_{B \in F_{2}} B C I-\sum_{i=1}^{m} F_{i}^{\prime}$ we infer

$$
\sum_{B \in F_{2}}\left|\phi\left[\mathfrak{M}_{A B} f_{N}\right]-\phi\left[\mathfrak{M}_{A B} f\right]\right||B| \leqq 2 M 3 \epsilon /(8 M)=3 \epsilon / 4,
$$

which completes the proof.

(10.9) THEOREM. The conditions $f \in R^{*}(I)$ and $\phi \in B C(\Re(f))$ are sufficient for (1.9), even with (1.6) replaced by (2.10).

Proof. Let $M$ satisfy the condition $|\phi(y)|<M<\infty$ for $y \in \Re(f)$; and let $\phi_{i}$ $(i=1,2,3, \cdots)$ be a normal approximating sequence for $\phi$ on $\Re(f)$, as defined in (5.5). Let sets $O_{i}, F_{i}$, and $F_{i}^{\prime}$ be defined as in the proof of Theorem (10.8). Let the positive integer $N$ satisfy the conditions

$$
\begin{aligned}
2 M\left|E_{N}\right|<\epsilon / 8 & \text { where } E_{N}=\underset{x}{E}[|f(x)|>N], \\
\underset{x \in F_{i}}{\operatorname{ess} \sup _{x}}|f(x)|<N & (i=1,2, \cdots, m) .
\end{aligned}
$$

Let $\delta_{1}=\delta_{1}\left(f, \phi_{N}, \epsilon / 8\right)>0$ correspond to $\epsilon / 8$ for the functions $f$ and $\phi_{N} \in B U C(\Re(f))$ in accordance with Theorem (10.8); let $\delta_{2}=\epsilon /(16 \mathrm{Mm})$; and let $\delta=\min \left[\delta_{1}, \delta_{2}\right]$. For $F \in \Gamma_{\delta}(I)$ and $F_{1}, F_{2}$ defined as in the proof of Theorem $(10.8)$ we then have

$$
\begin{aligned}
\sum_{B \in F}\left|\mathfrak{M}_{B} \phi: f-\phi\left[\mathfrak{M}_{A B} f\right]\right||B| \\
\leqq \int_{I}\left|\phi: f-\phi_{N}: f\right|+\sum_{B \in F}\left|\mathfrak{M}_{B} \dot{\phi}_{N}: f-\phi_{N}\left[\mathfrak{M}_{A B} f\right]\right||B| \\
\quad+\sum_{B \in F}\left|\phi_{N}\left[\mathfrak{M}_{A B} f\right]-\phi\left[\mathfrak{M}_{A B} f\right]\right||B| \\
<\epsilon / 8+\epsilon / 8+\left\{\sum_{B \in F_{1}}+\sum_{B \in F_{2}}\right\}\left|\phi_{N}\left[\mathfrak{M}_{A B} f\right]-\phi\left[\mathfrak{M}_{A B} f\right]\right||B|
\end{aligned}
$$


By the choice of $N$, each term in $\sum_{B \in F_{1}}$ vanishes and the sum $\sum_{B \in F_{2}}$ is not greater than $2 M 3 \epsilon /(8 M)=3 \epsilon / 4$.

11. Generalizations of boundedness, continuity, bounded variation, and absolute continuity of a function. The principal object of this section is to sketch in a background behind certain well known properties of the $L_{p}(p \geqq 1)$ classes of functions, which will throw these properties into rather clear relief. For our present purposes $E$ will be taken as the unit interval $I, f$ as a function whose domain includes $I$, and $\phi$ as a non-negative function whose domain includes the set of numbers

$$
[f(b)-f(a)] /(b-a), \quad \text { where } 0 \leqq a<b \leqq 1 .
$$

The results to be obtained will lose only a little of their significance if $\phi$ is regarded as a fixed function whose domain includes the interval $(-\infty, \infty)$, while the function $f$ is left free to vary; and this case is probably the one of greatest interest.

(11.2) Definition. The function $f$ shall be said to be $\phi$-bounded on $I$ if and only if there exists a number $M$ with

$$
\phi\left[\frac{f(b)-f(a)}{b-a}\right](b-a) \leqq M<\infty \quad \text { for } 0 \leqq a<b \leqq 1 .
$$

(11.3) Definition. For $a \in I$ the function $f$ shall be said to be $\phi$-continuous at $a$ if and only if the condition

$$
\lim _{x \in I, x \rightarrow a} \phi\left[\frac{f(x)-f(a)}{x-a}\right]|x-a|=0
$$

is satisfied; $f$ shall be said to be $\phi$-continuous on $I$ if and only if $f$ is $\phi$-continuous at each $a \in I$, uniformly $\phi$-continuous on $I$ if and only if condition (a) holds uniformly with respect to a for $a \in I$.

(11.4) Definition. The $\phi$-total variation of $f$ on $I$ is

$$
T_{I}^{\phi}(f)=\underset{\delta \rightarrow 0}{\lim \sup _{j}} \sum_{j=1}^{N} \phi\left[\frac{f\left(x_{j}\right)-f\left(x_{j-1}\right)}{x_{j}-x_{j-1}}\right]\left(x_{j}-x_{j-1}\right) \leqq \infty,
$$

where

$$
0=x_{0}<x_{1}<\cdots<x_{N}=1, \quad \delta=\max _{1 \leqq j \leqq N}\left(x_{j}-x_{j-1}\right) .
$$

If and only if $T_{I}^{\phi}(f)$ is $<\infty, f$ shall be said to be of $\phi$-bounded variation on $I$.

(11.5) Definition. The function $f$ shall be said to be $\phi$-absolutely continuous on $I$ if and only if to each $\epsilon>0$ corresponds $a \delta=\delta(\epsilon)>0$ such that, $\left[a_{j}, b_{j}\right]$ $\left(a_{i}<b_{j} ; j=1,2, \cdots, N\right)$ being any finite set of closed subintervals of $I$ disjoint except perhaps for common endpoints, the inequality $\sum_{j}\left(b_{j}-a_{j}\right)<\delta$ implies 


$$
\sum_{j} \phi\left[\frac{f\left(b_{j}\right)-f\left(a_{j}\right)}{b_{j}-a_{j}}\right]\left(b_{j}-a_{j}\right)<\epsilon .
$$

Several consequences of these definitions are plain. (I) The defining properties here employed reduce to those of ordinary boundedness, continuity, and so on when $\phi(y)=|y|$ for $-\infty<y<\infty$. (II) If $f$ is $\phi$-absolutely continuous on $I$, it is uniformly $\phi$-continuous on $I$ and of $\phi$-bounded variation on $I$. (III) If $\phi$ is bounded on the set of numbers (11.1), $f$ is $\phi$-absolutely continuous on $I$; in particular this hypothesis will be satisfied if $f$ satisfies a Lipschitz condition (of order 1) on $I$ with Lipschitz modulus $M$ and $\phi$ is bounded on the closed interval $[-M, M]$.

The observation that $\phi$ continuous on $(-\infty, \infty)$ and $f$ continuous and $\phi$-continuous on $I$ do not imply $f$ uniformly $\phi$-continuous on $I$ or $f \phi$-bounded on $I$ is justified by the following example. Let $0<l<\infty$ and $g(x)=l x$ for $x \in I$. Let $\left[a_{n}, b_{n}\right]\left(a_{n}<b_{n} ; n=1,2,3, \cdots\right)$ be an infinite sequence of disjoint closed subintervals of $I$ with the properties $b_{1}=1$,

$$
\lim _{n \rightarrow \infty} b_{n}=0 ; \quad b_{n+1}<a_{n}, \quad m_{n}+1 \leqq m_{n+1} \quad(n=1,2,3, \cdots),
$$

where

$$
m_{n}=\left[l b_{n}-\left(-l a_{n}\right)\right] /\left(b_{n}-a_{n}\right)=l\left(b_{n}+a_{n}\right) /\left(b_{n}-a_{n}\right) .
$$

Let

$$
f(x)=\left\{\begin{array}{rll}
0 & \text { for } & x=0, \\
l b_{n} & \text { for } x=b_{n}, \\
-l a_{n} & \text { for } x=a_{n}, \quad(n=1,2,3, \cdots),
\end{array}\right.
$$

and on each closed interval $\left[a_{n}, b_{n}\right],\left[b_{n+1}, a_{n}\right](n=1,2,3, \cdots)$ let $f$ be linear. Let $\phi \in C((-\infty, \infty))$ be arbitrary save for the satisfaction of the conditions

$$
\phi\left(m_{n}\right)=1 /\left(b_{n}-a_{n}\right) \quad(n=1,2,3, \cdots) .
$$

Then $f$ is easily seen to be continuous and $\phi$-continuous on $I$ but not uniformly $\phi$-continuous on $I$. If $p_{n}(n=1,2,3, \cdots)$ is any infinite sequence of numbers with

$$
m_{n}<p_{n}<m_{n+1} \quad(n=1,2,3, \cdots),
$$

it is clear that $\phi$ can be so defined on the points $p_{n}$ (with $\lim _{n \rightarrow \infty} \phi\left(p_{n}\right)=\infty$ ) that $f$ will not be $\phi$-bounded on $I$.

In the subsequent discussion we shall have occasion at times to subject $\phi$ to one or more of the following conditions: continuity (usually on $-\infty<y<\infty$ ), convexity (usually on $-\infty<y<\infty$ ),

$$
\lim _{|y| \rightarrow \infty} \inf \phi(y) /|y|>0 \text {, }
$$




$$
\lim _{|y| \rightarrow \infty} \phi(y) /|y|=\infty,
$$

the last two being suggested, respectively, by the particular cases $\phi(y)=|y|$, $\phi(y)=|y|^{p}(p>1)$ on $-\infty<y<\infty$.

The function $\phi(y)=|y|^{p}(p>1)$ is convex and satisfies (11.7). For this function $\phi$ it is well known $\left(^{8}\right)$ that $\phi$-bounded variation of $f$ implies $\phi$-absolute continuity of $f$, so that the two conditions are equivalent; also that a necessary and sufficient condition for $f$ to be the indefinite integral of a function $g$ with $g \in L_{\phi}=L_{p}$ is that $f$ be $\phi$-absolutely continuous. It seems to us of some interest to determine (1) whether these properties are enjoyed by a more general class of functions $L_{\phi}$, and (2) whether convexity of $\phi$ or the satisfaction of (11.7) by $\phi$ is the more important contributing factor in bringing about these properties.

One may easily prove

(11.8) LEMma. The condition (11.6) is equivalent to the condition that there exist positive numbers $a, b$ with $a+b \phi(y) \geqq|y|$ for $-\infty<y<\infty$.

Using this lemma one obtains at once

(11.9) ThEOREM. If $\phi$ satisfies (11.6) and $f$ is $\phi$-bounded [or $\phi$-continuous, or of $\phi$-bounded variation, or $\phi$-absolutely continuous] on $I, f$ is bounded [or continuous, or of bounded variation, or absolutely continuous, respectively] on $I$.

More generally, in fact, if $\phi_{1}, \phi_{2}$ are such that there exist positive numbers $a, b$ with $a+b \phi_{1}(y) \geqq \phi_{2}(y)$ for $-\infty<y<\infty, \phi_{1}$-boundedness or $\phi_{1}$-continuity or and so on of $f$ on $I$ implies the corresponding $\phi_{2}$-property of $f$ on $I$.

(11.10) THEOREM. If $\phi$ satisfies (11.7) and $f$ is of $\phi$-bounded variation on $I$, $f$ is absolutely continuous on $I$.

Proof. Let $T_{I}^{\phi}(f)=B$; let $\delta_{1}>0$ be such that

$$
\sum_{j=1}^{N} \phi\left[\frac{f\left(x_{j}\right)-f\left(x_{j-1}\right)}{x_{j}-x_{j-1}}\right]\left(x_{j}-x_{j-1}\right)<2 B \quad \text { for } \delta<\delta_{1} ;
$$

let $M=M(\epsilon)>0$ be such that $3 B / M<\epsilon$; and let $Q=Q(M)>0$ be such that

$$
\phi(y) /|y|>M \quad \text { for }|y| \geqq Q \text {. }
$$

Then we have

$$
M Q+\phi(y)>M|y| \quad \text { for }-\infty<y<\infty .
$$

If the norm $\delta$ of the intervals $\left[a_{j}, b_{j}\right]$ is $<\delta_{1}$, we have

(8) See, for example, Titchmarsh, The theory of functions, Oxford, 1932, pp. 384-386. 


$$
\begin{aligned}
2 B & >\sum_{j} \phi\left[\frac{f\left(b_{j}\right)-f\left(a_{j}\right)}{b_{j}-a_{j}}\right]\left(b_{j}-a_{j}\right) \\
& >\sum_{j}\left[M\left|f\left(b_{j}\right)-f\left(a_{j}\right)\right|-M Q\left(b_{j}-a_{j}\right)\right] \\
& =M \sum_{j}\left|f\left(b_{j}\right)-f\left(a_{j}\right)\right|-M Q \sum_{j}\left(b_{j}-a_{j}\right) ;
\end{aligned}
$$

and if $\sum_{j}\left(b_{j}-a_{j}\right)$ is $<B /(M Q)$, we obtain

and

$$
M \sum_{j}\left|f\left(b_{j}\right)-f\left(a_{j}\right)\right|<2 B+M Q B /(M Q)=3 B,
$$

$$
\sum_{j}\left|f\left(b_{j}\right)-f\left(a_{j}\right)\right|<3 B / M<\epsilon .
$$

REMARK. If $\phi$ is convex on $(-\infty, \infty)$ and satisfies (11.7), and $f$ is absolutely continuous and $\phi$-continuous on $I$, it does not follow that $f$ is uniformly $\phi$-continuous or of $\phi$-bounded variation on $I$. This is shown by the example $\phi(y)=y^{2}$ on $(-\infty, \infty)$,

$$
f(x)=\left\{\begin{array}{cl}
1 / 2^{n} & \text { for } x=1 / 2^{n}, \\
0 & \text { for } x=1 / 2^{n} \pm 1 / 2^{2 n}, \\
0 & \text { for } x=0,1 \\
\text { linear } \text { on each closed interval between consecutive points for } \\
\text { which } f \text { is already defined. }
\end{array}\right.
$$

(11.11) THEOREM. If $\phi$ is continuous on the closure of the set of numbers (11.1) and $f^{\prime}$ exists ( finite) almost everywhere in $I$, we have $\int_{I} \phi: f^{\prime} \leqq T_{I}^{\phi}(f) \leqq \infty$.

Proof. Let $S_{n}(n=1,2,3, \cdots)$ represent a sequence of points of subdivision of $I$,

$$
S_{n}: \quad 0=x_{n, 0}<x_{n, 1}<\cdots<x_{n, N_{n}}=1,
$$

with

$$
\begin{gathered}
\lim _{n \rightarrow \infty} \delta_{n}=0, \quad \delta_{n}=\max _{1 \leqq j \leqq N_{n}}\left(x_{n, j}-x_{n, j-1}\right), \\
T_{I}^{\phi}(f)=\lim _{n \rightarrow \infty} \sum_{j=1}^{N_{n}} \phi\left[\frac{f\left(x_{n, j}\right)-f\left(x_{n, j-1}\right)}{x_{n, j}-x_{n, j-1}}\right]\left(x_{n, j}-x_{n, j-1}\right) .
\end{gathered}
$$

For each $n$ let $p_{n}$ stand for the polygonal function inscribed in $f$ with $p_{n}(x)=f(x)$ for $x \in S_{n}$, so that we have

$$
T_{I}^{\phi}(f)=\lim _{n \rightarrow \infty} \int_{I} \phi: p_{n}^{\prime} .
$$


Since $f^{\prime}$ exists almost everywhere, $p_{n}^{\prime}$ tends to $f^{\prime}$ almost everywhere $\left(^{9}\right)$ in $I$. The function $\phi$ being continuous and non-negative on the closure of the set of numbers (11.1), a well known theorem of Fatou yields

$$
\int_{I} \phi: f^{\prime} \leqq \lim _{n \rightarrow \infty} \int_{I} \phi: p_{n}^{\prime} \leqq \infty,
$$

the desired conclusion.

REMARK. The hypothesis of continuity on $\phi$ cannot be deleted from this theorem. For the example $\phi$ non-measurable on $I, f(x)=x^{2} / 2$ for $x \in I$, shows that $\int_{I} \phi: f^{\prime}$ may not then exist. And the example $\phi(y)=1$ for $-\infty<y \leqq 0$, $\phi(y)=0$ for $0<y<\infty, f$ a non-decreasing singular function constant on no subinterval of $I$, yields $\int_{I} \phi: f^{\prime}=1>0=T_{I}^{\phi}(f)$.

(11.12) THEOREM. If $f$ is absolutely continuous on $I$ and $\phi \in C\left(\Re\left(f^{\prime}\right)\right)$, the condition $S^{*}\left(f^{\prime}, \phi, I\right)<\infty$ implies that $f$ is $\phi$-absolutely continuous on $I$ and $\int_{I} \phi: f^{\prime}=T_{I}^{\phi}(f)<\infty$.

Proof. The hypotheses imply the existence of $\delta$ satisfying the conditions

$$
0<\delta \leqq 1, \quad \sup _{F \in \Gamma_{\delta}(I)} \sum_{B \in F} \phi\left[\mathfrak{M}_{B} f^{\prime}\right]|B|<\infty .
$$

For $b_{j}-a_{j}<\delta(j=1,2,3, \cdots)$ we have

$$
\begin{aligned}
0 & \leqq \sum_{j} \phi\left[\frac{f\left(b_{j}\right)-f\left(a_{j}\right)}{b_{j}-a_{j}}\right]\left(b_{j}-a_{j}\right)=\sum_{j} \phi\left[\int_{a_{j}}^{b_{j}} f^{\prime} /\left(b_{j}-a_{j}\right)\right]\left(b_{j}-a_{j}\right) \\
& \leqq \sup _{F \in \Gamma_{\delta}(H)} \sum_{B \in F} \phi\left[\mathfrak{M}_{B} f^{\prime}\right]|B|,
\end{aligned}
$$

where

$$
H=\sum_{j}\left[a_{j}, b_{j}\right]
$$

By Theorem (4.4), this sup tends to zero with $|H|$; hence the first conclusion follows. The hypotheses also imply, by Theorem (5.11),

$$
\begin{aligned}
\int_{I} \phi: f^{\prime} & =\lim _{\delta \rightarrow 0} \sum_{j=1}^{N} \phi\left[\int_{x_{j-1}}^{x_{j}} f^{\prime} /\left(x_{j}-x_{j-1}\right)\right]\left(x_{j}-x_{j-1}\right) \\
& =\lim _{\delta \rightarrow 0} \sum_{j=1}^{N} \phi\left[\frac{f\left(x_{j}\right)-f\left(x_{j-1}\right)}{x_{i}-x_{j-1}}\right]\left(x_{j}-x_{j-1}\right)=T_{I}^{\phi}(f)<\infty .
\end{aligned}
$$

From Theorem (5.13) we obtain the

Corollary. If $f$ is absolutely continuous on I and $\phi \in C\left(\Re\left(f^{\prime}\right)\right)$ is dominated by a convex function $\psi$ with $\int_{I} \psi: f^{\prime}<\infty$, the same conclusions may be drawn.

(') See, for example, Titchmarsh, loc. cit. pp. 385-386. 
Another immediate consequence of Theorem (5.11) is

(11.13) THEOREM. Under the hypotheses of Theorem (11.12) or its Corollary, if $k$ is $\geqq 1$ and the intervals $\left[a_{j}, b_{j}\right]$ satisfy the conditions

$a_{j}, b_{j} \in\left[x_{j-1}, x_{j}\right], \quad 0<x_{j}-x_{j-1} \leqq k\left(b_{j}-a_{j}\right) \quad(j=1,2, \cdots, N)$, we have

$$
T_{I}^{\phi}(f)=\lim _{\delta \rightarrow 0} \sum_{j=1}^{N} \phi\left[\frac{f\left(b_{j}\right)-f\left(a_{j}\right)}{b_{j}-a_{j}}\right]\left(x_{j}-x_{j-1}\right) .
$$

The next theorem is an application of Theorem (10.9).

(11.15) THEOREM. If $f$ is absolutely continuous on $I$ with $f^{\prime} \in R^{*}(I)$, $\phi \in B C\left(\Re\left(f^{\prime}\right)\right)$, and the intervals $\left[a_{j}, b_{i}\right]$ satisfy the conditions

$$
a_{j}, b_{j} \in\left[x_{j-1}, x_{j}\right], \quad 0<b_{j}-a_{j} \quad(j=1,2, \cdots, N),
$$

we have (11.14).

The next three theorems are immediate consequences of Theorems (11.9), (11.10), and (11.11).

(11.16) THEOREM. If $\phi$ is continuous on $(-\infty, \infty)$ and satisfies (11.6), a sufficient condition for $f^{\prime} \in L_{\phi}(I)$ is that $f$ be of $\phi$-bounded variation on $I$.

Proof. By Theorem (11.9), $f$ is of bounded variation on $I$ and $f^{\prime}$ consequently exists (finite) almost everywhere in $I$; since $f$ is of $\phi$-bounded variation, Theorem (11.11) yields $\int_{I} \phi: f^{\prime} \leqq T_{I}^{\phi}(f)<\infty$.

Similarly we obtain

(11.17) THEOREM. If $\phi$ is continuous on $(-\infty, \infty)$ and satisfies (11.6), a sufficient condition for

$$
f^{\prime} \in L_{\phi}(I), \quad f(x)=f(0)+\int_{0}^{x} f^{\prime}(t) d t \text { for } x \in I,
$$

is that $f$ be $\phi$-absolutely continuous on $I$.

(11.19) ThEOREM. If $\phi$ is continuous on $(-\infty, \infty)$ and satisfies (11.7), a sufficient condition for (11.18) is that $f$ be of $\phi$-bounded variation on $I$.

Proof. By Theorem (11.10), $f$ is absolutely continuous on $I$; and from Theorem (11.11) we have $\int_{I} \phi: f^{\prime} \leqq T_{I}^{\phi}(f)<\infty$.

That no one of the sufficient conditions given in Theorems (11.16),(11.17), and (11.19) is necessary is shown by Example B of $\S 8$. For, let the function called $f$ in that example be designated now by $f^{\prime}$ and let $f$ be defined on $I$ by

$$
f(x)=\int_{0}^{x} f^{\prime}(t) d t \quad \text { for } x \in I .
$$


Defining $\phi(y)=16+y^{2}$ for $-\infty<y<0$, we obtain a non-negative $\phi$ which is continuous everywhere and satisfies (11.7). And although $f$ is absolutely continuous on $I$, with $f^{\prime} \in L_{\phi}(I)$, it is not of $\phi$-bounded variation on $I$ in virtue of inequality (8.1).

The following example achieves the same result in a different way, exhibiting a non-negative $\phi$ which is continuous everywhere and satisfies (11.7), and an $f$ which is absolutely continuous on $I$, with $f^{\prime} \in L_{\phi}(I)$, but has an "infinite $\phi$-discontinuity" at $x=0$.

Let $g(x)=x^{2 / 3}$ for $x \in I$, so that $g^{\prime} \in L_{2}(I)$. Let $0 \leqq a<b \leqq 1$; and let $h(x)$ for $x \in[a, b]$ be linear with $h(a)=g(a), h(b)=g(b)$. Simple computations then yield

$$
\begin{aligned}
\int_{a}^{b}\left[h^{\prime}\right]^{2} & =\left[1+\frac{(a b)^{1 / 3}}{a^{2 / 3}+(a b)^{1 / 3}+b^{2 / 3}}\right]\left(b^{1 / 3}-a^{1 / 3}\right)<2\left(b^{1 / 3}-a^{1 / 3}\right) \\
& <(8 / 3)\left(b^{1 / 3}-a^{1 / 3}\right)=2 \int_{a}^{b}\left[g^{\prime}\right]^{2} .
\end{aligned}
$$

In view of the fact that $g$ is concave on $I$ with $g^{\prime}$ increasing continuously and monotonically toward $\infty$ as $x$ decreases from 1 toward 0 , there exists a sequence of numbers $a_{n}(n=1,2,3, \cdots)$ decreasing monotonically toward 0 and satisfying the following conditions:

$$
\begin{aligned}
& a_{1}=1, \\
& 0<a_{2}<a_{1}, \quad l_{1}=\frac{g\left(a_{1}\right)-g\left(a_{2}\right)}{a_{1}-a_{2}}<\frac{g\left(a_{1}\right)}{a_{1}}=m_{1}=1, \\
& 0<a_{n+1}<a_{n}, \quad m_{n-1}<l_{n}=\frac{g\left(a_{n}\right)-g\left(a_{n+1}\right)}{a_{n}-a_{n+1}}<\frac{g\left(a_{n}\right)}{a_{n}}=m_{n} \\
& \quad(n=2,3,4, \cdots) .
\end{aligned}
$$

Each of the sequences $\left\{l_{n}\right\},\left\{m_{n}\right\}$ then increases monotonically toward $\infty$ with $n$ and between each pair of consecutive numbers from either sequence occurs one and only one number from the other sequence. Hence there exists a sequence of closed intervals $\left\{I_{n}\right\}$ with the following property: for each $n, I_{n}$ has $m_{n}$ as its midpoint and $I_{n}$ contains neither $l_{n}$ nor $l_{n+1}$. That is, the set $\sum_{n=1}^{\infty} I_{n}$ contains no point of the sequence $\left\{l_{n}\right\}$.

Let $f$ be defined on $I$ as follows: $f(0)=0$; for each $n, f(x)$ is linear on the interval $\left[a_{n+1}, a_{n}\right]$ with $f\left(a_{n+1}\right)=g\left(a_{n+1}\right), f\left(a_{n}\right)=g\left(a_{n}\right)$. From inequality (11.20) we infer $f^{\prime} \in L_{2}(I)$. Since $f$ is monotone and continuous on $I$, and on each interval $[\epsilon, 1](0<\epsilon<1)$ it is polygonal and so absolutely continuous, it is absolutely continuous on $I$.

For $y$ not in $\sum_{n=1}^{\infty} I_{n}$, let $\phi(y)=y^{2} ;$ for $y=m_{n}(n=1,2,3, \cdots)$, let $\phi(y)=y^{4}$; in each closed half (that is, left and right half) of each interval $I_{n}$, let $\phi$ be 
linear. Then $\phi$ is continuous for $-\infty<y<\infty$ and satisfies (11.7). We also have, by aid of (11.20),

$$
\int_{I} \phi: f^{\prime}=\sum_{n=1}^{\infty} \int_{a_{n+1}}^{a_{n}} \phi: f^{\prime}=\sum_{n=1}^{\infty} \int_{a_{n+1}}^{a_{n}}\left[f^{\prime}\right]^{2}<2 \sum_{n=1}^{\infty} \int_{a_{n+1}}^{a_{n}}\left[g^{\prime}\right]^{2}=2 \int_{I}\left[g^{\prime}\right]^{2}=8 / 3 \text {. }
$$

On the other hand if $x$ tends to 0 over the sequence $\left\{a_{n}\right\}$, we have

$$
\begin{gathered}
f\left(a_{n}\right) / a_{n}=a_{n}^{2 / 3} / a_{n}=1 / a_{n}^{1 / 3}=m_{n}, \quad \phi\left(m_{n}\right)=m_{n}^{4}=1 / a_{n}^{4 / 3}, \\
\phi\left[\frac{f\left(a_{n}\right)-f(0)}{a_{n}-0}\right]\left(a_{n}-0\right)=\phi\left(m_{n}\right) a_{n}=1 / a_{n}^{1 / 3} \rightarrow \infty .
\end{gathered}
$$

From the Corollary to Theorem (5.13) we obtain at once

(11.22) THEOREM. If $f$ is absolutely continuous on $I$ and $\phi$ is convex and continuous on $\Re\left(f^{\prime}\right)$, we have $\int_{I} \phi: f^{\prime}=T_{I}^{\phi}(f) \leqq \infty$.

Using Theorem (11.12), we obtain the

CoRollary. If $f$ is absolutely continuous on $I, \phi$ is convex and continuous on $\Re\left(f^{\prime}\right)$, and $f$ is of $\phi$-bounded variation on $I$, then $f$ is $\phi$-absolutely continuous on $I$.

Theorem (11.22) implies that if $f$ is absolutely continuous on $I$ and $\phi$ is convex and continuous on $\Re\left(f^{\prime}\right)$, we have

$$
T_{I}^{\phi}(f)=\lim _{\epsilon \rightarrow 0} T_{[\epsilon, 1]}^{\phi}(f) \leqq \infty \quad(0<\epsilon<1) .
$$

That the assumption of convexity on $\phi$ is essential for (11.23) may be seen from the following example, which exhibits an $f$ which is absolutely continuous on $I$ and a $\phi$ which is continuous everywhere and satisfies (11.7), but $f$ has a "finite $\phi$-discontinuity" at $x=0$, with $\lim \sup _{x \rightarrow 0} \phi[(f(x)-f(0)) / x] x=1$; and for each positive $\epsilon<1$

$$
\begin{gathered}
f \text { is } \phi \text {-absolutely continuous on }[\epsilon, 1], \\
T_{[\epsilon, 1]}^{\phi}(f)<4 \cdot 2^{1 / 2}, \quad T_{I}^{\phi}(f)=T_{[0, \epsilon]}^{\phi}(f)=\infty .
\end{gathered}
$$

Let $g(x)=x^{1 / 2}$ for $x \in I$, so that $g^{\prime} \in L_{3 / 2}(I)$. Let $0 \leqq a<b \leqq 1$; and let $h(x)$ for $x \in[a, b]$ be linear with $h(a)=g(a), h(b)=g(b)$. Simple computations provide the inequality

$$
\begin{aligned}
\int_{a}^{b}\left[h^{\prime}\right]^{3 / 2} & =\frac{b^{1 / 4}+a^{1 / 4}}{\left(b^{1 / 2}+a^{1 / 2}\right)^{1 / 2}}\left(b^{1 / 4}-a^{1 / 4}\right)<2\left(b^{1 / 4}-a^{1 / 4}\right) \\
& =2^{1 / 2} \int_{a}^{b}\left[g^{\prime}\right]^{3 / 2}
\end{aligned}
$$


As in the example last described, $g$ is concave on $I$ with $g^{\prime}$ increasing continuously and monotonically toward $\infty$ as $x$ decreases from 1 toward 0 , and there exists a sequence of numbers $\left\{a_{n}\right\}$ satisfying conditions (11.21). This time let $I_{n}(n=1,2,3, \cdots)$ be a sequence of closed intervals with the following property: for each $n, I_{n}$ has $l_{n}$ as its midpoint and $I_{n}$ contains neither $m_{n-1}$ nor $m_{n}$.

Let $f$ be defined on $I$ as in the preceding example, so that it is absolutely continuous there. From (11.24) it is clear that $f^{\prime} \in L_{3 / 2}(I)$. For $y$ not in $\sum_{n=1}^{\infty} I_{n}$, let $\phi(y)=y^{2}$; for $y=l_{n}(n=1,2,3, \cdots)$, let $\phi(y)=y^{3 / 2}$; in each closed half (that is, left and right half) of each interval $I_{n}$, let $\phi$ be linear. Thus $\phi$ is continuous for $-\infty<y<\infty$ and satisfies (11.7).

On the interval $[\epsilon, 1], f$ satisfies a Lipschitz condition; let $M=M(\epsilon)$ satisfy the inequality

$$
\left|f\left(x_{1}\right)-f\left(x_{2}\right)\right| \leqq M\left|x_{1}-x_{2}\right| \quad \text { for } x_{1}, x_{2} \in[\epsilon, 1] .
$$

On the interval $[0, M], \phi$ is bounded; hence $\phi$ is dominated on the interval $(-\infty, \infty)$ by a convex function $\psi$, with $\int_{\epsilon}^{1} \psi: f^{\prime}<\infty$. From the corollary to Theorem (11.12) we therefore infer the $\phi$-absolute continuity of $f$ on $[\epsilon, 1]$ and, in view of (11.24) also, the relation

$$
T_{[\epsilon, 1]}^{\phi}(f)=\int_{\epsilon}^{1} \phi: f^{\prime}=\int_{\epsilon}^{1}\left[f^{\prime}\right]^{3 / 2}<\int_{I}\left[f^{\prime}\right]^{3 / 2}<2^{1 / 2} \int_{I}\left[g^{\prime}\right]^{3 / 2}=4 \cdot 2^{1 / 2} .
$$

For $0<x \leqq 1$ we also have $f(x) / x \geqq 1$ and

$$
\phi[f(x) / x] x \leqq[f(x) / x]^{2} x \leqq[g(x) / x]^{2} x=1,
$$

whence

$$
\limsup _{x \rightarrow 0} \phi[f(x) / x] x \leqq 1 ;
$$

and if $x$ tends to 0 over the sequence $\left\{a_{n}\right\}$, we have

$$
\begin{aligned}
& f\left(a_{n}\right) / a_{n}=g\left(a_{n}\right) / a_{n}=a_{n}^{1 / 2} / a_{n}=1 / a_{n}^{1 / 2}=m_{n}, \quad \phi\left(m_{n}\right)=m_{n}^{2}=1 / a_{n}, \\
& \phi\left[f\left(a_{n}\right) / a_{n}\right] a_{n}=1 \quad(n=1,2,3, \cdots),
\end{aligned}
$$

which implies

$$
\limsup _{x \rightarrow 0} \phi[f(x) / x] x=1 .
$$

To show the property $T_{[0, \epsilon]}^{\phi}=\infty \quad(0<\epsilon<1)$, we choose a particular sequence $\left\{a_{n}\right\}$; namely $a_{n}=1 / 2^{2(n-1)}(n=1,2,3, \cdots)$. This clearly satisfies the principal inequality of (11.21),

$$
g\left(a_{n-1}\right) / a_{n-1}<\left[g\left(a_{n}\right)-g\left(a_{n+1}\right)\right] /\left(a_{n}-a_{n+1}\right)<g\left(a_{n}\right) / a_{n},
$$

which reduces to 


$$
1 / a_{n-1}^{1 / 2}<1 /\left(a_{n}^{1 / 2}+a_{n+1}^{1 / 2}\right)<1 / a_{n}^{1 / 2}
$$

since we now have

$$
a_{n}^{1 / 2}+a_{n+1}^{1 / 2}=1 / 2^{n-1}+1 / 2^{n}=\left(1 / 2^{n-2}\right)(1 / 2+1 / 4)<1 / 2^{n-2}=a_{n-1}^{1 / 2} .
$$

From the obvious inequality

$$
\left[g\left(a_{n}\right)-g\left(a_{n+2}\right)\right] /\left(a_{n}-a_{n+2}\right)<g\left(a_{n}\right) / a_{n}=m_{n},
$$

we infer that a line drawn from the midpoint $P_{n+1}$ of the segment of $f$ standing over the interval $\left[a_{n+2}, a_{n+1}\right]$, with slope $m_{n}$, meets the segment of $f$ over $\left[a_{n+1}, a_{n}\right]$ at a point $Q_{n}$ to the left of the midpoint $P_{n}$ of that segment. Thus if $c_{n}, d_{n}$ are the abscissae of $P_{n+1}, Q_{n}$, respectively, it is clear that the intervals $\left[c_{n}, d_{n}\right](n=1,2,3, \cdots)$ are disjoint, with

$$
\left(a_{n}-a_{n+2}\right) / 2>d_{n}-c_{n}>a_{n+1}-c_{n}=\left(a_{n+1}-a_{n+2}\right) / 2 .
$$

But for each $n$ we have

$$
\begin{gathered}
{\left[f\left(d_{n}\right)-f\left(c_{n}\right)\right] /\left(d_{n}-c_{n}\right)=m_{n}=g\left(a_{n}\right) / a_{n}=1 / a_{n}^{1 / 2}=2^{n-1},} \\
\phi\left(m_{n}\right)=m_{n}^{2}=2^{2(n-1)}, \\
\phi\left(m_{n}\right)\left(d_{n}-c_{n}\right)>\phi\left(m_{n}\right)\left(a_{n+1}-a_{n+2}\right) / 2=2^{2(n-1)}\left(1 / 2^{2 n}-1 / 2^{2(n+1)}\right) / 2=3 / 32 .
\end{gathered}
$$

Since $d_{n}-c_{n}$ is $<\left(a_{n}-a_{n+2}\right) / 2$, which tends to 0 with $1 / n$, and the intervals $\left[c_{n}, d_{n}\right](n=1,2,3, \cdots)$ are disjoint, it follows that in any interval $[0, \epsilon]$, and for an arbitrary norm $\delta>0$, the sum whose lim sup is $T_{[0, e]}^{\phi}(f)$ can be made arbitrarily large by a suitable choice of points of subdivision of $[0, \epsilon]$.

We conclude with two more theorems which show that well known properties of the $L_{p}(p \geqq 1)$ classes of functions hold for certain more general $L_{\phi}$ classes.

(11.25) THEOREM. If $\phi$ is convex on $(-\infty, \infty)$ and satisfies (11.6), a necessary and sufficient condition for (11.18) is that $f$ be $\phi$-absolutely continuous on $I$.

The sufficiency of the condition follows from Theorem (11.17), the necessity from the Corollary to Theorem (11.12).

(11.26) THEOREM. If $\phi$ is convex on $(-\infty, \infty)$ and satisfies (11.7), a necessary and sufficient condition for (11.18) is that $f$ be of $\phi$-bounded variation on $I$.

The sufficiency is a consequence of Theorem (11.19); the necessity follows from Theorem (11.22).

Brown UNIVERSITY, Providence, R. I.

The University of California,

Berkeley. Calif. 